NISTIR 7650

\title{
High-Speed Dual-Spectrum Imaging for the Measurement of Metal Cutting Temperatures (2010 edition)
}
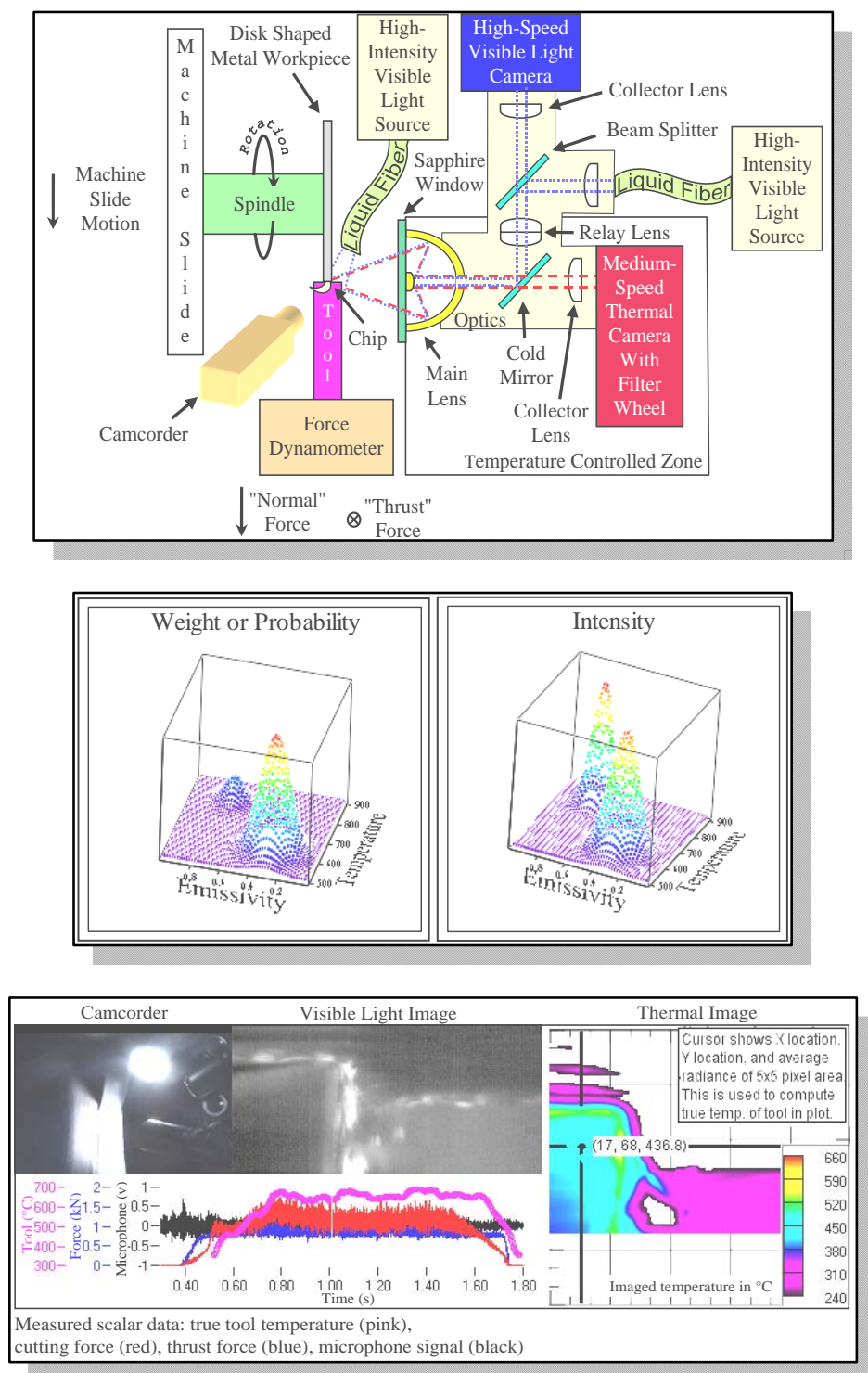

Eric P. Whitenton
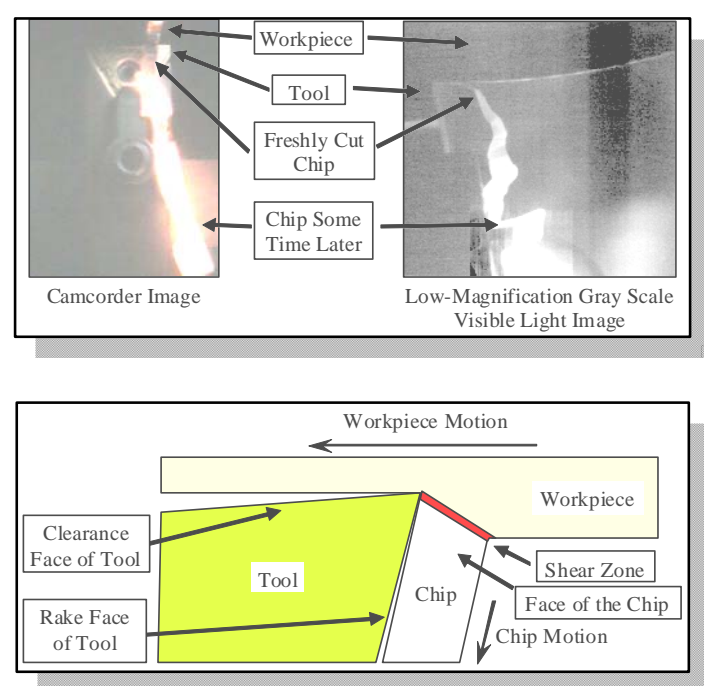

National Institute of Standards and Technology U.S. Department of Commerce 
This work was generated by the Fundamental Metrology for Material Processing Project, part of the Measurements and Standards for Science-Based Manufacturing Program of MEL (Manufacturing Engineering Laboratory) at NIST (National Institute of Standards and Technology). It is available in electronic format at www.nist.gov.

\section{The Fundamental Metrology for Material Processing Project}

This project works to develop advanced process metrology methods and tools to increase scientific understanding of existing, as well as new and emerging discrete part manufacturing processes. Industry interactions and priorities determine specific application case studies as appropriate. Several process parameters and phenomena are expected to be of critical importance for multiple manufacturing processes, such that common and generic process metrology approaches can be devised. These process phenomena include forces, temperatures, bulk material properties, and material transformations at the material/tool interface, tool wear and performance, friction considerations, system vibrations and dynamic response of the manufacturing process.

\section{The MEL Measurements and Standards for Science-Based Manufacturing Program}

To remain competitive in the global marketplace, U.S. manufacturing is currently implementing fundamental changes, focused on designing and building complex, highly-customized, high quality goods -- all built speedily to meet the rapidly changing demands of the market. Manufacturers must shorten product development cycles and increase flexibility and speed of production systems and supply networks. And they must do all this while also reducing environmental impacts and energy requirements. These changes require a transformation from manufacturing practices based on human experience towards scientific-based modeling, decision making, and production. This program develops fundamental measurements, standards, and tools to enable U.S. manufacturers to make this transformation. 
NISTIR 7650

\section{High-Speed Dual-Spectrum Imaging for the Measurement of Metal Cutting Temperatures (2010 edition)}

Eric P. Whitenton Manufacturing Engineering Laboratory

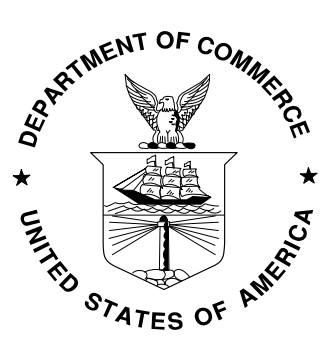

U.S. Department of Commerce Gary Locke, Secretary 



\title{
High-Speed Dual-Spectrum Imaging for the Measurement of Metal Cutting Temperatures (2010 edition)
}

\author{
Eric P. Whitenton \\ National Institute of Standards and Technology, Gaithersburg, MD USA 20899-8223
}

\begin{abstract}
Models are important tools for optimizing the metal cutting process, allowing industry to make parts faster, better, and at less cost. Measurements of the process using thermal and visible imaging can be used to improve and verify the accuracy of these models. There are many error sources when using infrared radiation thermography to measure the temperature distribution of the tool, workpiece, and chip during metal cutting. It is important to understand how these error sources affect the measurement uncertainty. Some error sources are familiar to anyone performing thermography measurements, such as uncertainties in the basic camera calibration. However, metal cutting presents unique measurement challenges due to factors such as the high magnification required, high surface speeds, polarization effects, micro-blackbody effects, and changing emissivity as the metal is cut.

MADMACS, the MAnufacturing Deformation MACro videography System, developed by MEL (Manufacturing Engineering Laboratory) at NIST (National Institute of Standards and Technology), consists of a high-speed visible light camera, a medium-speed thermal camera, and a common primary lens so the two cameras are imaging the same scene. A data acquisition system records timing information for the cameras, as well as other signals such as cutting forces. Researchers may review the images and signals synchronized to each other.

This report focuses on thermal spectrum measurements, though visible spectrum measurements are also shown. The report is not intended to be an exhaustive list of all possible uncertainty sources, but shows many qualitative examples of the sources that can occur. Some examples illustrate very basic concepts, while others are more complex. Images of a number of cutting experiments with different cutting conditions and materials are shown. For brevity, the specifics of the cutting experiments are not given, as this report has a measurement focus. The two primary goals of this report are to discuss the issues involved when performing thermal imaging of the metal cutting process and also describe MADMACS to assist those who may wish to either use MADMACS or build a similar system.
\end{abstract}

Keywords: uncertainty, metal cutting, dual-spectrum, high-speed imaging, infrared thermography

Disclaimer: This document is an official contribution of the National Institute of Standards and Technology and is not subject to copyright in the United States. The National Institute of Standards and Technology does not recommend or endorse commercial equipment or materials, nor does it imply that the equipment or materials shown are necessarily the best available for the purpose. 


\section{TABLE OF CONTENTS}

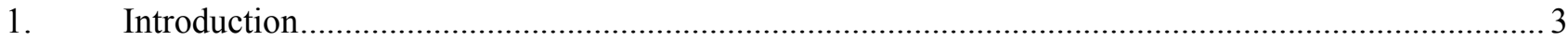

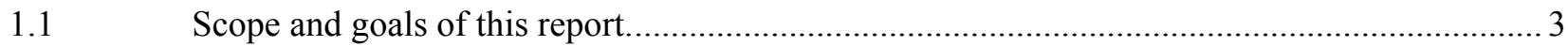

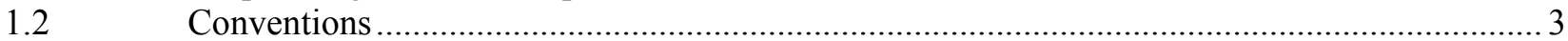

$1.3 \quad$ Why measure metal cutting temperatures?

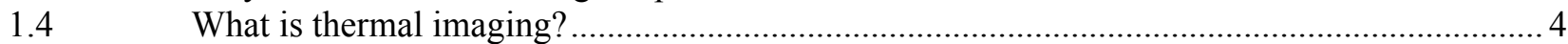

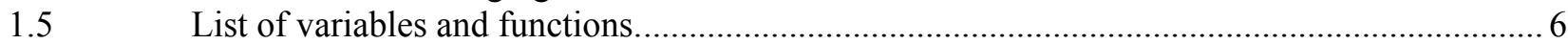

1.6 Early work at NIST - learning the need for dual-spectrum imaging ........................................ 7

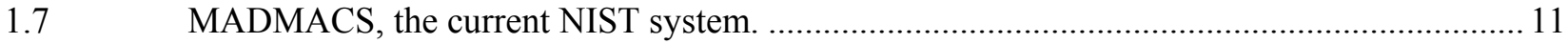

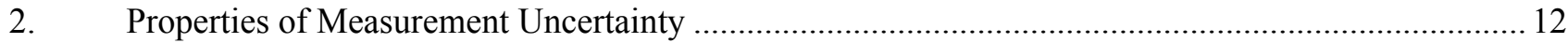

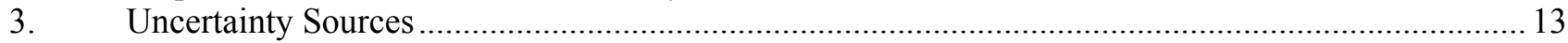

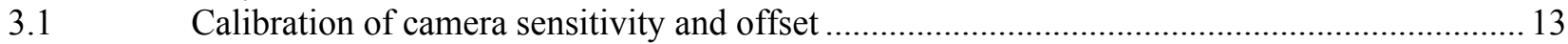

Example 1: Emissivity of the calibration source ..................................................................... 13

Example 2: Use of an appropriate aperture .......................................................................... 14

Example 3: Understanding resolution and repeatability ....................................................... 16

Example 4: The range of linear operation .............................................................................. 17

Example 5: Sensitivity to polarization ……………………………………………………..... 18

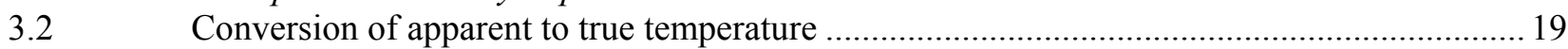

Example 6: The form of the equation used to convert to true temperature............................... 19

Example 7: Nonuniform surface textures. …………………………………………….... 20

Example 8: Oxide layer changes …………………………………………………….... 23

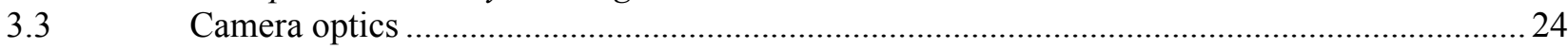

Example 9: Optical pixel crosstalk ............................................................................... 25

Example 10: Optical size-of-source effects ……………………………………………… 25

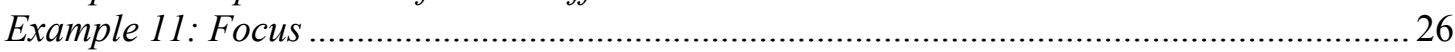

Example 12: Distortion …………………………………………………………….... 27

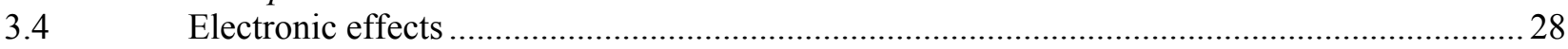

Example 13: Electronic pixel cross talk …………………………………………….... 28

Example 14: Nonuniformity ………………………………………………………… 28

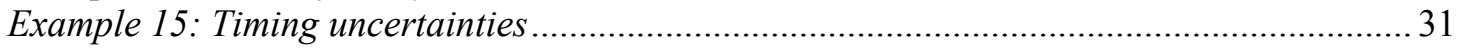

Example 16: Persistence, ………………………………………………………… 32

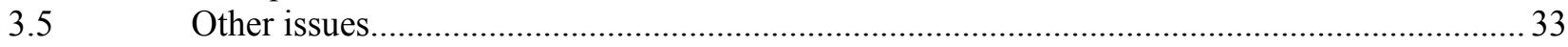

Example 17: Reflections …………………………………………………………..... 33

Example 18: Are surface temperatures an accurate measure of internal temperatures? …......34

Example 19: Motion blur, size-of-source effects, and insufficient temperature range.............. 35

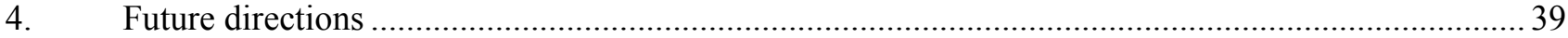

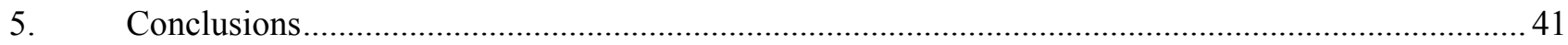

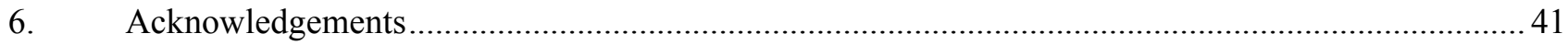

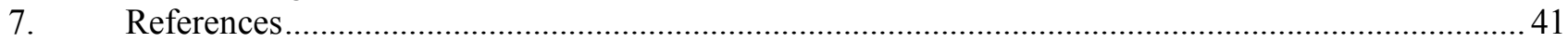

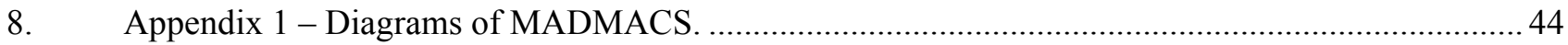

9. $\quad$ Appendix 2 - Nomograms for MADMACS............................................................................... 48

10. Appendix 3 - Mathcad ${ }^{\circledR}$ document to compute error due to nonuniform temperature and emissivity in thermal images which are motion blurred. ..............................................................................5

11. Appendix 4-Mathcad $\mathbb{B}$ document to correct shear band temperature measurements.........................56 


\section{INTRODUCTION}

\subsection{Scope and goals of this report.}

As with any measurement system, there are sources of uncertainty that must be understood to fully assess the quality of measurement results. This report groups sources of uncertainty into five categories: calibration of camera sensitivity and offset, conversion of apparent to true temperature, camera optics, electronic effects, and other issues. Some uncertainties are familiar to anyone trying to perform radiation thermography measurements, such as the need to calibrate the camera and to take emissivity into account. However, metal cutting is especially challenging due to factors such as the high magnifications, high surface speeds, and ever changing emissivity of the metal as chips form. Each source of uncertainty may be of greater or lesser importance depending on the specific quantity measured. For example, when measuring how much the work piece heats up, reflections, camera sensitivity, and pixel cross talk are of major concern. By contrast, when measuring shear zone temperature, size-of-source and motion blur effects are generally more important.

MADMACS, the MAnufacturing Deformation MACro videography System, developed by MEL (Manufacturing Engineering Laboratory) at NIST (National Institute of Standards and Technology), consists of a high-speed visible light camera, a medium-speed thermal camera, and a common primary lens so the two cameras image the same scene. A data acquisition system records timing information for the cameras, as well as other signals such as cutting forces. The images and signals may be viewed synchronized to each other.

This report focuses on thermal spectrum measurements, though visible spectrum measurements are also shown. It is not intended to be an exhaustive list of all possible uncertainty sources, but this report does show many qualitative and quantitative examples of the main sources. Some examples illustrate well understood uncertainty sources, while other examples illustrate less well known uncertainty sources. Images of a number of cutting experiments with different cutting conditions and materials are shown. For brevity, the specifics of the cutting experiments are not given, as this paper has a measurement focus. Three goals of manufacturing-related research at NIST are: develop and improve measurement techniques, develop an understanding of the uncertainties involved with performing such measurements, and compare models of machining to thermal and visible spectrum images to verify the models. The goal of this report is to directly and indirectly contribute to all three of these goals by educating the machining community in general, informing NIST collaborators how to correctly interpret data produced by MADMACS, and providing guidance to others who may wish to build similar systems.

\subsection{Conventions}

In general, the first time an important concept is discussed in this paper it is presented in bold italic. Variable names are presented in italic, and are defined in Section 1.5. The symbol @ means "corresponding to."

\subsection{Why measure metal cutting temperatures?}

Modeling is an important tool for optimizing the metal cutting process, allowing industry to make parts faster, better, and at less $\operatorname{cost}^{(1)}$. One type of model is a Finite Element Model, or FEM. By comparing measurements of the process to model predictions, the models may be improved and verified. There are many types of relevant measured data which may be compared, such as cutting forces or vibrations. Thermal ${ }^{(2 ; 3)}$ and visible ${ }^{(4 ; 5)}$ imaging provide a wealth of useful data, such as temperatures and strains, which may be directly compared to modeling results. 
Figure 1 shows a schematic of a typical image of an orthogonal cutting process. The field of view is about a millimeter wide. The relative motion between a cutting tool and a workpiece causes material to be removed from the workpiece. This removed material is referred to as a chip. Both the workpiece and the chip move at high speeds, making them a challenge to image. Most of the deformation of the workpiece occurs within a thin area called the shear zone, which can be as thin as only a few micrometers. Two of the many types of chips are called continuous and segmented. A continuous chip is a long ribbon of relatively uniformly deformed material. By contrast, a segmented chip has alternating zones of low and high shear strain. A zone of low strain in a segmented chip is called a segment. The zones of high strain between the segments are mechanically weak, so the long ribbons of material tend to break into short pieces. These short pieces are more manageable than a continuous chip. Even when segmented chips do not break completely, there is generally a partial gap between the segments. The shear zone is somewhat stationary when continuous chips are formed, but often travel along with the chips when the chips are segmented. We will call the area surrounding the shear zone the face of the chip. During cutting, the shear zone has a higher temperature than the face. For segmented chips, the shear zone generally also has a higher emissivity than the face. A chip often has some areas of side flow, where metal has flowed toward the camera during machining and protrudes beyond the side of the chip.

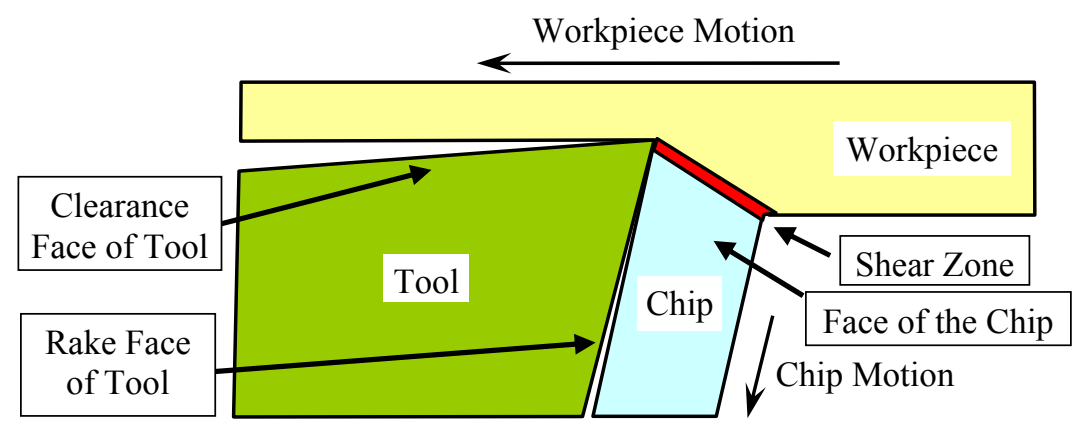

Fig. 1. Schematic of an image of an orthogonal cutting process.

\subsection{What is thermal imaging?}

Thermal radiation is electromagnetic radiation emitted by an object related to the object's temperature. A range of wavelengths, or colors, are emitted. The temperature of the object, along with other factors, determines the intensity, or brightness, at each wavelength. Radiation thermography is the use of a sensor to measure the thermal radiation emitted by an object, generally with the intent to use this information to determine the object's temperature. Sensors often contain only a single sensing element and yield a single measurement value. By contrast, a thermal camera uses a focal plane array (FPA), which is an array of sensors. By displaying the array of measured values, an image is formed which is a representation of the temperature distribution on the surface of the object. Each element of the array is a pixel. Each pixel corresponds to a location in space of the scene being imaged, called a scenel. The path traveled by the light as it propagates from the object being imaged to the FPA is the optical path of the system.

True temperature refers to the actual temperature of an object. Apparent temperature refers to the temperature reported by a "perfect" camera, and includes properties of the scene being imaged such as emissivity and reflections. Note that this is not necessarily the same as the imaged temperature, which is the temperature reported by the actual camera, and also includes properties of the image acquisition process such as scattering in the camera optics. It is important to remember that thermal cameras do not actually 
measure temperature. They measure intensity of electromagnetic radiation within a range of wavelengths during a period of time (the integration time of the camera). The calibration of the camera allows a user to convert these measured intensities into imaged temperatures. Imaged temperatures may be converted into true temperatures if one has a qualitative and quantitative understanding of the physical properties of the objects, the characteristics of the camera, as well as the conditions encountered while acquiring the images. Attaining accurate true temperatures is generally the goal when using thermal cameras.

The wavelengths of light being detected depend on the spectral response of the camera and any filters used. The FPA in the visible light camera is sensitive to wavelengths shorter than $1 \mu \mathrm{m}$, while the thermal camera is sensitive to wavelengths between $3.6 \mu \mathrm{m}$ to $5.1 \mu \mathrm{m}$. However, the optical components in the system limit the thermal camera response to $3.8 \mu \mathrm{m}$ to $5.1 \mu \mathrm{m}$. As will be discussed in Example 6, it is common to use a filter to limit this even further to make the conversion from apparent temperature to true temperature easier. However, this requires much longer integration times to accommodate the decreased intensity, which increases motion blur. Motion blur occurs when the object in an image moves more than 1 pixel in that image during the integration time. For example, a small, stationary object, say only a few pixels in size, might appear as a dot in the image. However, if the object is not stationary and moves 10 pixels during the integration time, the light from each location on the object will be distributed across 10 pixels. This causes what looks like streaks which are 10 pixels long. As discussed in Example 19, since the light is distributed across 10 pixels, the intensity often appears less bright than if all the light fell on just 1 pixel.

Spatial resolution may be thought of as either the size of the smallest object in space one can detect or as the smallest space between objects one can detect. Spatial resolution is affected by several factors. One factor is scenel spacing, the distance between scenels, since one can not easily detect objects smaller than 1 scenel. Another factor, optical resolution, is determined primarily by the way light interacts with the optical system of the camera. Optical resolution is not accurately described by a single number, but as a curve such as a point spread function (PSF). One way of thinking of a PSF is to imagine the camera is focused on a single point of light which would fill exactly 1 pixel of the FPA if the camera optics were perfect. In this case, the FPA would report the 1 bright pixel, with all neighboring pixels having an intensity value of 0 . Unfortunately, even when the image is in focus, real optics spread this point over an area. The curve describing how the light is spread is the PSF. The wavelength of light affects optical resolution; shorter wavelengths can resolve smaller features than longer wavelengths. Thus, all else being equal, the visible light camera will yield a sharper image than the thermal camera. Temporal resolution is often thought of as the shortest event in time one can detect. It is affected by both the integration time of the camera and the frame rate (the number of images acquired per unit time). Intensity resolution is the smallest change in light intensity one can detect. It is affected by factors such as noise in the FPA, and the fact that intensities are digitized and converted into digital levels, or DLs (discussed in Example 3). Field of view is the width and height of the scene being imaged. This is typically about $1 \mathrm{~mm}$ for MADMACS. Depth of field is the distance toward or away from the camera an object being imaged may move and still produce an acceptable image. Depth of field is dependent on the magnification used and the wavelength of light imaged.

A blackbody is an object or material that completely absorbs all incident radiation, and emits uniform thermal radiation which is the maximum possible (is perfectly efficient) as described by Planck's Law. Any body which is perfectly efficient in emitting thermal radiation is said to have an emissivity of 1 . Most objects have an emissivity less than 1. Emissivity is an important characteristic of an object when converting apparent temperature into true temperature. For the same true temperature, the apparent temperature will increase with emissivity. When machined, emissivity of the cut metal is very nonuniform and changes dramatically due to changes in surface texture and oxidation. Many researchers feel that this may be the single most difficult source of error to correct when measuring temperatures of machining. A micro-blackbody is a very small feature, such as a small deep hole or narrow deep crevasse, which has a 
very high emissivity. Machining chips often have micro-blackbodies. This contrasts with a properly conditioned cutting tool, which has relatively small changes in tool emissivity.

There are other considerations besides those mentioned above. Many published works exist describing thermal imaging in general $^{(6 ; 7)}$, as well as procedures for converting intensities into true temperatures.

\subsection{List of variables and functions.}

Excluding the appendices, the following variables and functions are used this paper:

$A$ - Diameter of an aperture.

$C$ - Total camera response.

$c_{1 \mathrm{~L}}$ and $c_{2}-$ First radiation constant $\left(119.1 \mu \mathrm{W} \cdot \mu \mathrm{m}^{2} / \mathrm{sr}\right)$ and second radiation constant $(14388 \mu \mathrm{m} \cdot \mathrm{K})$.

$D$ - Length of the diagonal of the field of view.

$E_{\mathrm{e}}-$ Error due to electrical effects such as voltage offsets.

$E_{\mathrm{o}}$ - Error due to optical effects such as infrared light emitted by the optical system.

$E_{\mathrm{c}}$ - Combined error due to electronic and optical effects.

$F$ - Distance from the lens to an in-focus object being imaged. This is about $25 \mathrm{~mm}$ for MADMACS.

$I$ - Intensity of light emitted by the object being imaged.

$I_{\mathrm{CF}}$ - Intensity of light at the chip face.

$I_{\mathrm{SB}}$ - Intensity of light at the shear band.

$j$-Current image (or frame) in a series of images (a movie).

$k$-Coverage factor used for calculating the expanded uncertainty.

$L-$ Distance from an aperture to some other object.

$M$ - A variable to minimize by adjusting other variables.

$P$ - Diameter of the exit pupil of the lens.

$R(I)$ - A function describing the response of the FPA to light intensity.

$S$ - Safety factor, 1.2 is a generally a good value.

$T_{\text {apparent }}$ - Apparent temperature of the target as measured by a perfect camera.

$T_{\text {environment }}$ - Temperature of the surrounding environment.

$T_{\text {true }}$ - True temperature of the target.

$W_{\mathrm{MB}}$ - Width of motion blur. (How far the object moved during the integration time of the camera.)

$W_{\mathrm{SB}}-$ Width of the shear band.

$w_{\lambda}$ - Relative sensitivity of the camera at wavelength $\lambda$.

$\varepsilon-$ Emissivity of the target. Ranges from 0 to 1 .

$\lambda$ - Wavelength of light.

$\lambda_{1}$ and $\lambda_{2}-$ Shortest and longest wavelength the camera is sensitive to. 


\subsection{Early work at NIST - learning the need for dual-spectrum imaging}

Machining is a dynamic process. Cutting tools may vibrate, chips may intermittently obscure the camera's view, and material flow and shear zone may vary. In the early stages of work at NIST, there was concern that thermal camera images alone lack sufficient spatial and temporal resolution to unambiguously image the process. Figure 2 shows a relatively simple apparatus designed to assess the severity of the problem by providing visible light images of the process. A conventional camcorder is used to image the chips through a macro lens or a microscope lens attachment. The video output is converted to a once per frame pulse using a video signal converter purchased from a video equipment supply house. The once per frame pulse triggers an inexpensive strobe/tachometer unit commonly used in machine shops. The room lights need to be off or the machine needs to be covered so that the only light the camcorder sees is that of the strobe.

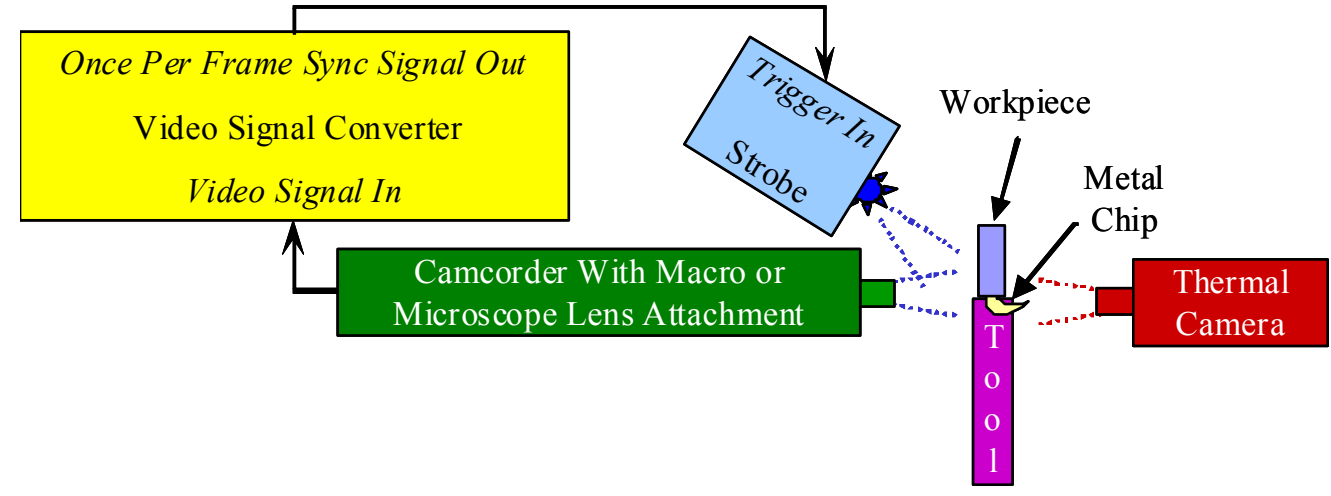

Fig. 2. Relatively simple apparatus for gauging the quality of thermal images.

When set up properly, this system outputs a surprisingly good quality movie. While the frame rate is far too low to study chip dynamics, if the entire range of cutting conditions of interest are imaged with this setup, and all of the images look good, then the thermal images will likely be good as well. However, it was noticed that there are many frames which were compromised. Examples are shown in Figure 3. Even when the quality of thermal images is good, the low frame rate of the thermal camera means that many aspects of the cutting process can not be captured and studied ${ }^{(8)}$.

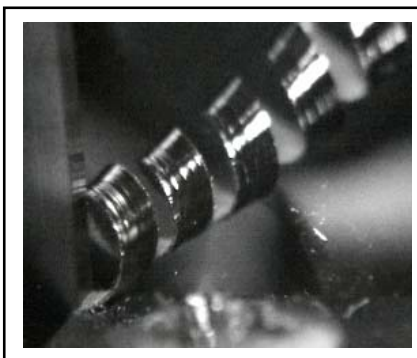

Expect an acceptable thermal image.

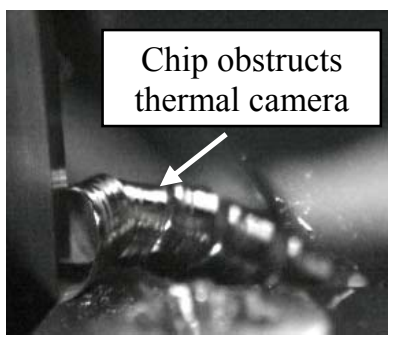

Expect a compromised thermal image.

Floppy chip intermittently blocks thermal camera view.

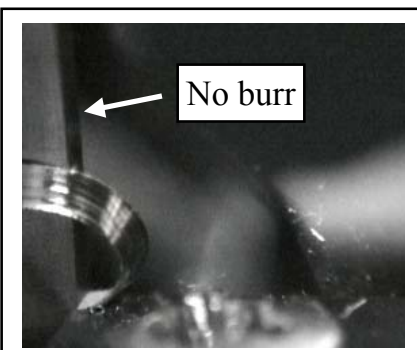

Expect an acceptable thermal image.

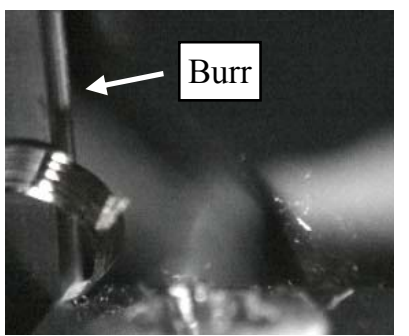

Expect a compromised thermal image.
Burr on workpiece intermittently blocks thermal camera.

Fig. 3. Low magnification visible light imaging of machining helps gauge the quality of thermal images. 
It was next decided to build a rudimentary dual-spectrum system which combines a thermal and a high-speed visible light camera. Shown in Figure 4, both cameras see the exact same scene because they share a common primary lens. However, the high-speed visible light camera often has a significantly better temporal and spatial resolution than the thermal camera. The visible light images yield information on the material flow and chip geometry while the thermal camera yields information on the temperatures.

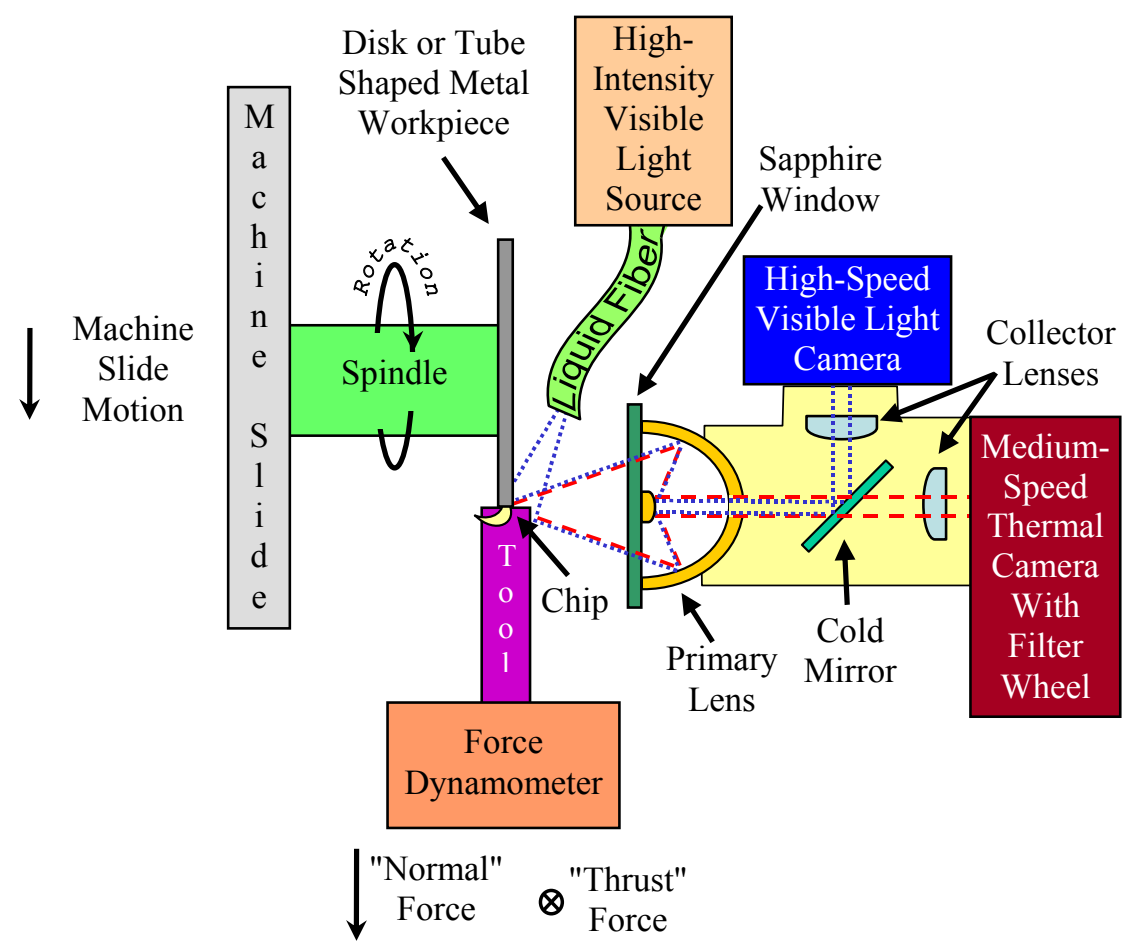

Fig. 4. Rudimentary dual-spectrum system used to measure machining processes. Both cameras share a common primary lens.

The primary lens is a reflective lens that transmits both visible and infrared light. The cold mirror reflects visible light to the visible light camera and transmits thermal radiation to the thermal camera.

Shown in Figure 5, the images are aligned by imaging a well defined target visible to both cameras and adjusting the position of the optical elements.

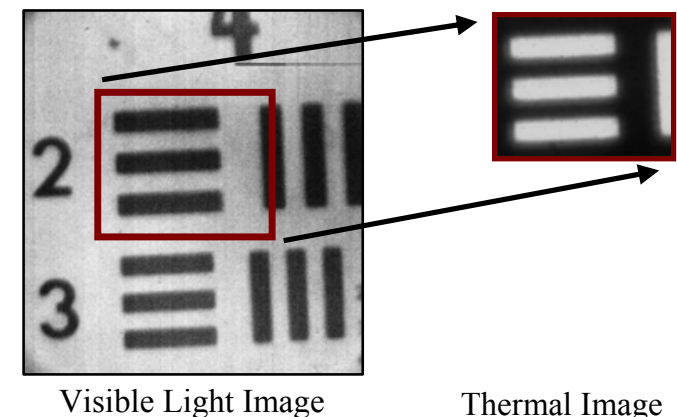

Fig. 5. Alignment of the visible and thermal images by imaging a well defined target. In this case, a United States Air Force (USAF) 1951 target. 
Use of the dual-spectrum camera revealed numerous situations where thermal images alone could easily be misinterpreted. Figures 6 and 7 show two of these.

In Figure 6, the effect of "pig tails" on thermal images is shown. A pig tail occurs when material flows towards the camera, perpendicular to the chip flow direction. Pig tails move much slower than the chip, and often occur when the tool is worn. The visible/thermal image pair shown in Figure 6a shows a good imaged temperature measurement of the chip/tool interface. The presence of a pig tail is obvious in the center thermal image (Figure 6b), but not obvious in the lower thermal image (Figure 6c) without the accompanying visible light image. Since a pig tail moves at a much slower speed than the chip, the high frame rate of the visible light movies makes it easy to differentiate a pig tail from a chip. The speed at which an object moves is difficult to assess in thermal movies due to the relatively low frame rate of thermal movies. Note the $35^{\circ} \mathrm{C}$ difference between the top (good) and the bottom (aberrant) thermal images.

a

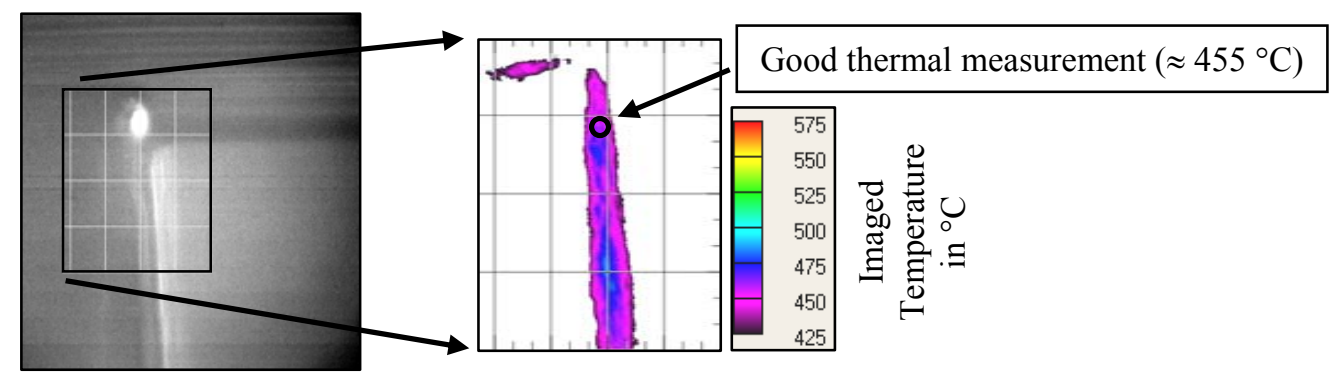

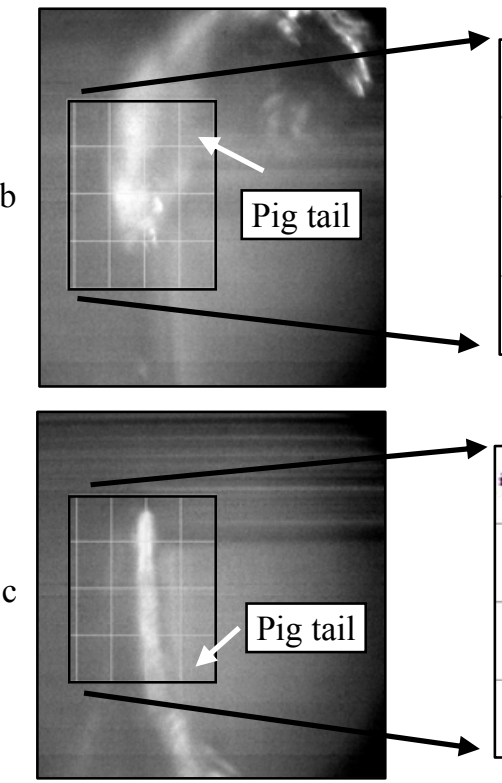

Visible Light Image

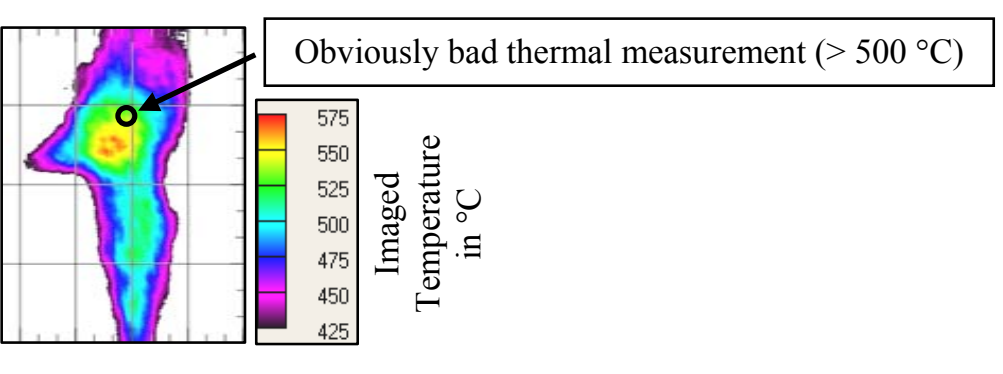

Non-obvious bad thermal measurement $\left(\approx 490^{\circ} \mathrm{C}\right)$

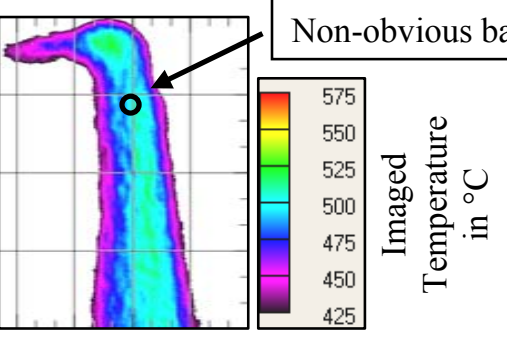

Thermal Image

Fig. 6. Pigtails, which adversely affect the accuracy of chip temperature measurements, are not always obvious in the thermal images. However, they are easily detected in the visible light images. Visible light images have about a $2 \mathrm{~mm}$ wide field of view and the thermal images have about a $1 \mathrm{~mm}$ wide field of view. 
A built up edge is a lump of material which has built up on the cutting edge of the tool, forcing the chip to flow over it. Shown in Figure 7, this built up edge can create a gap between the chip and the tool that can behave like a micro-blackbody. This causes this area of the image to have an emissivity very different from the emissivity of either the tool or the chip. If not taken into account, the higher than expected emissivity of the gap results in the derived true temperatures being positively biased (too high).

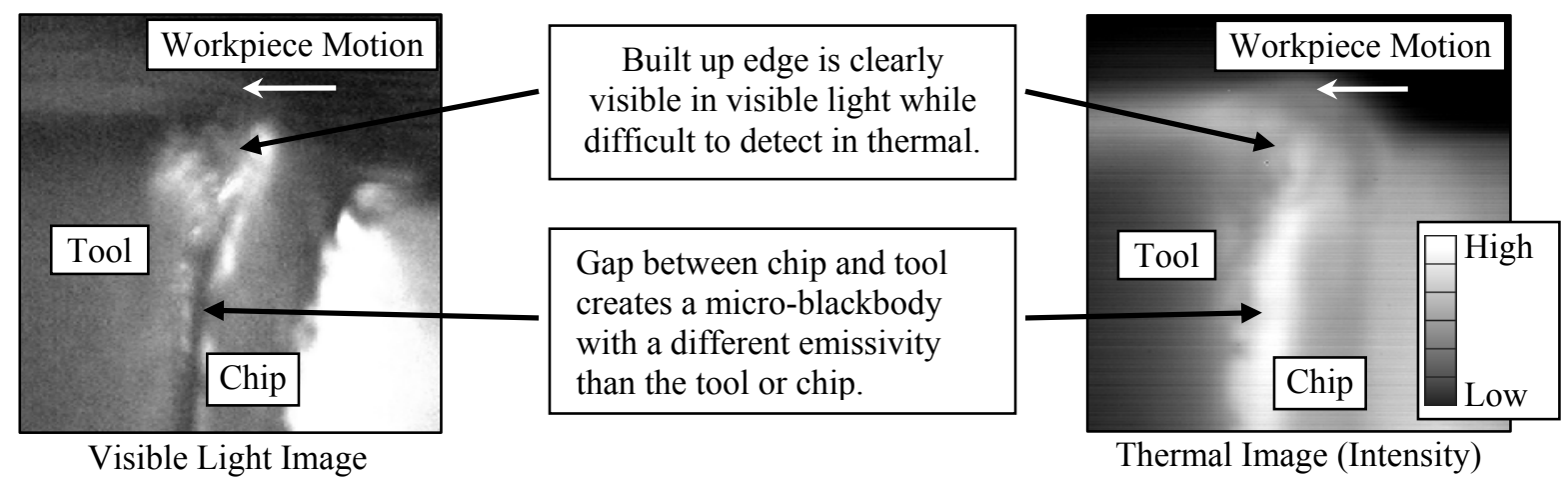

Fig. 7. Image pairs from the rudimentary dual-spectrum system. About a $1 \mathrm{~mm}$ wide field of view.

Note that the configuration of the machine tool (Figure 4) allows for the camera to be stationary, reducing camera vibration. Also note that early research sometimes machined the end off of a hollow cylinder and sometimes machined the edge off of a disk. Machining a disk is closer to orthogonal cutting than cutting a tube, and has less of a tendency for the chips to go between the workpiece and camera.

The side of the tool, workpiece, and chip are typically imaged as the chip is formed. There is a range of camera settings used, depending on cutting conditions and the machining phenomena being investigated. Generally, the images have about a $1 \mathrm{~mm}$ wide field of view. The visible spectrum images are typically acquired at 30000 frames per second, yielding 256 pixel by 128 pixel images. The workpiece and chip are typically traveling between $0.5 \mathrm{~m} / \mathrm{s}$ and $5 \mathrm{~m} / \mathrm{s}$. To minimize motion blur, integration time is adjusted to the shortest possible. This is determined primarily by the power of the lighting system available. Under some lighting conditions, the integration times can be long (about $33 \mu \mathrm{s}$ ). With sufficient lighting, the integration times can be less than $1 \mu \mathrm{s}$. The thermal spectrum images are typically acquired at 300 frames per second, yielding 160 pixel by 120 pixel images. Integration time is adjusted to minimize motion blur while maintaining enough sensitivity to image temperatures of interest. This typically results in integration times between $9 \mu \mathrm{s}$ and $20 \mu \mathrm{s}$. A data acquisition system records the timing of the thermal and visible spectrum images, along with analog data such as cutting forces. Custom software allows all of the data streams to be played back in a synchronized manner. 


\subsection{MADMACS, the current NIST system.}

Over time, improvements have been incorporated into the system. Figure 8 shows the current system, dubbed MADMACS.

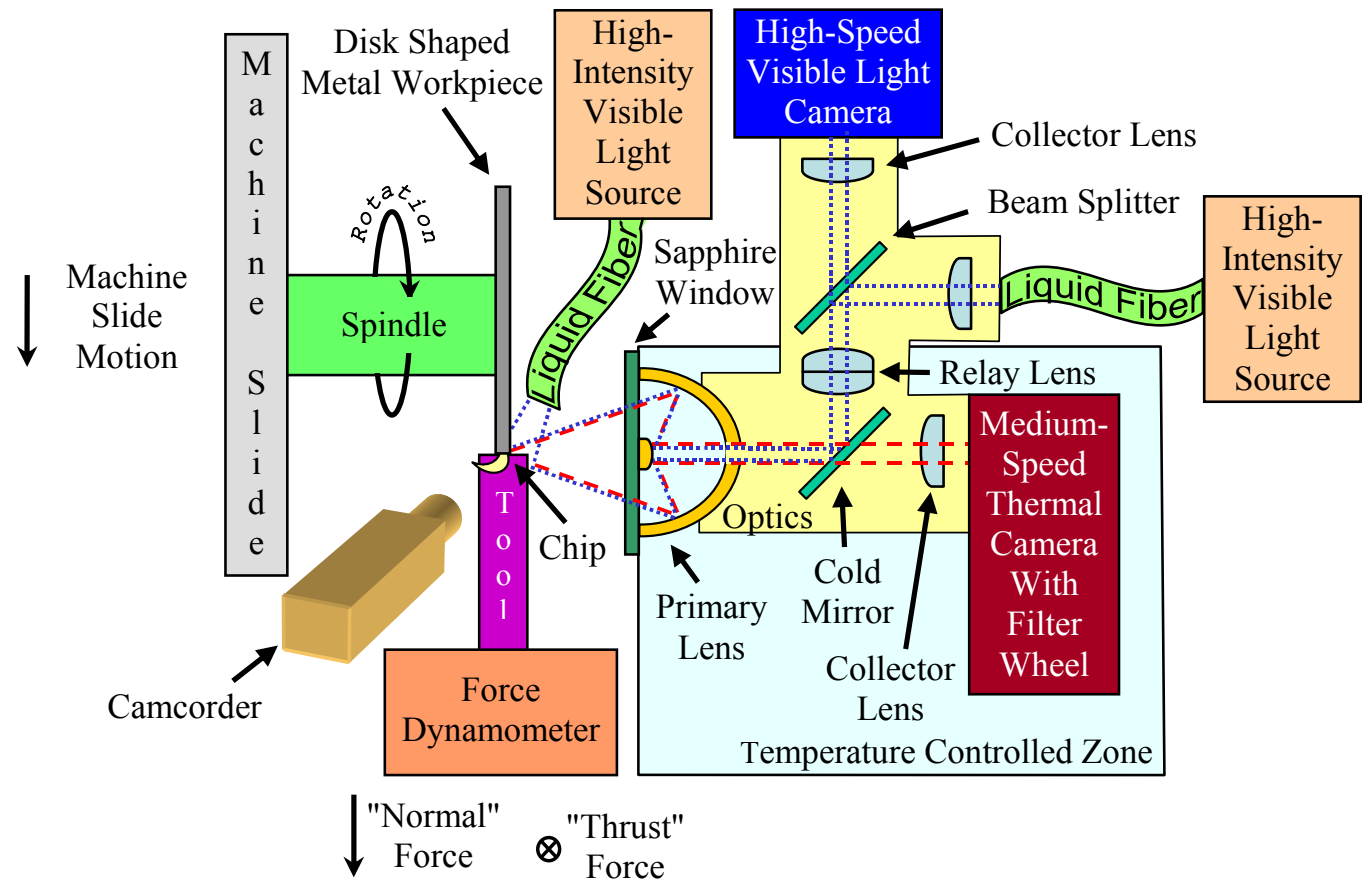

Fig. 8. The current system. Chilled, temperature-controlled water is circulated to stabilize the temperature of the optics. Through-the-lens lighting aids positioning of the camera system. Low magnification camcorder gives an overview of the machining process.

Chilled, temperature-controlled water is circulated to stabilize the temperatures of the optics. This makes the thermal images less susceptible to drift. Through-the-lens lighting aids positioning of the camera system by projecting a spot of visible light onto the object being imaged. At an angle to the dual-spectrum system is a conventional camcorder which images the scene at a low magnification. An example of a camcorder video is shown in Figure 9, and is available on the NIST website ${ }^{(9)}$. Various schematic diagrams of the system are shown in Appendix 1.

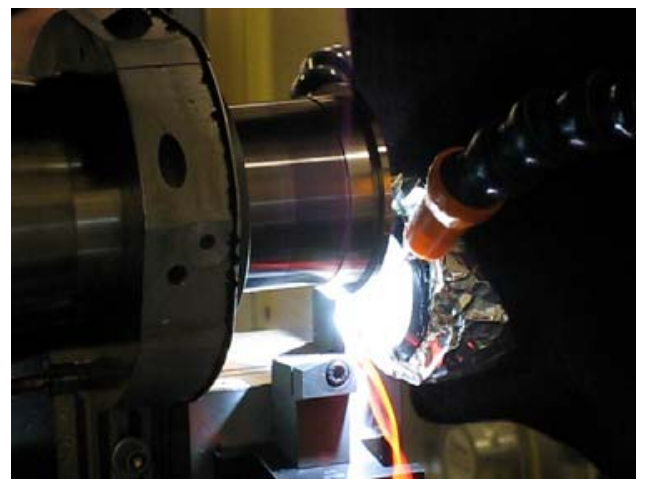

Fig. 9. Typical machining test. 
Figure 10 shows a typical data set. In this example, the true temperature at a given location on the tool as a function of time is computed. Such a rich data set facilitates many aspects of machining research ${ }^{(10)}$.

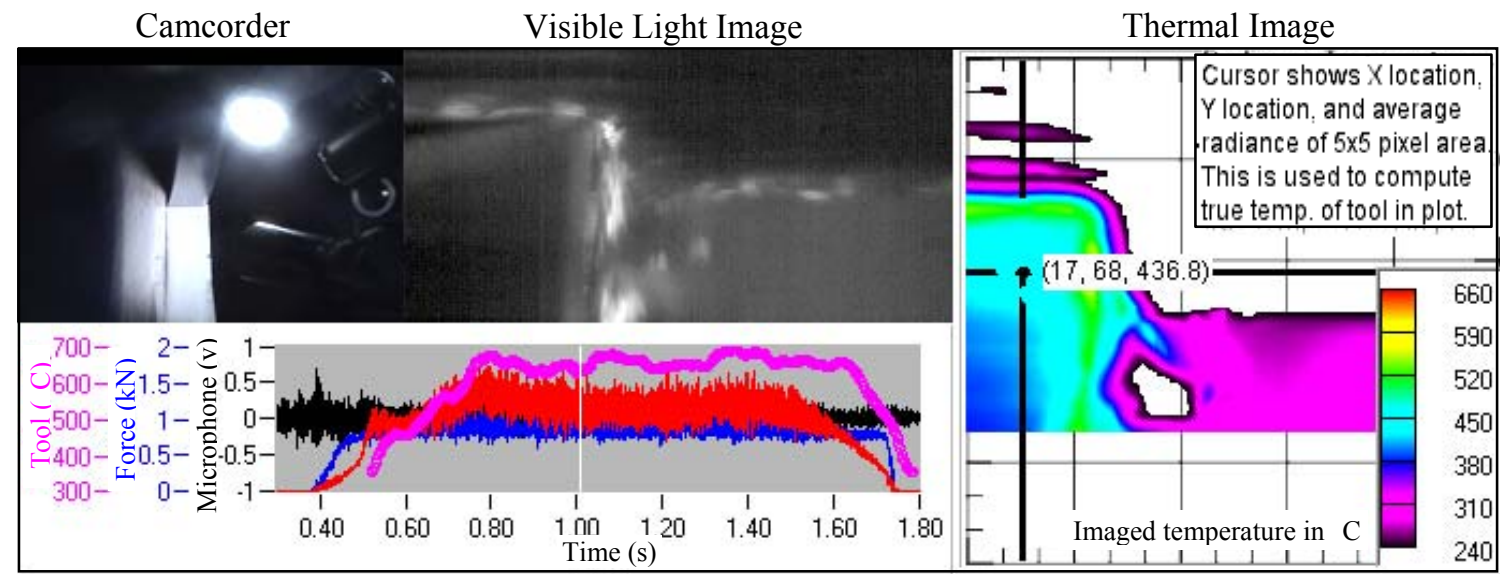

Measured scalar data: true tool temperature (pink),

cutting force (red), thrust force (blue), microphone signal (black)

Fig. 10. Typical synchronized dataset. Here, the imaged temperature of the tool is used to compute the true temperature of the tool as a function of time.

Recently, other dual-spectrum schemes have appeared in the literature ${ }^{(11)}$. Any scheme will have a unique set of characteristics, such as the maximum frame rate attainable, the number of frames captured, and the inherent image quality. The cutting conditions and materials studied by NIST are industrially relevant. If a parameter, such as surface speed, is too fast and causes the images to be inaccurate, NIST works to either improve the imaging system or learn how to "make do" with the images which are attainable. Early work by some researchers to image machining used very slow cutting conditions. Unfortunately, this yields high quality images of cutting conditions which do not address the needs of industry.

\section{PROPERTIES OF MEASUREMENT UNCERTAINTY}

It is important to remember that any temperature measurement is valid only at some location during some time interval. Thus, to fully gauge uncertainties associated with a measurement, one may need to consider three different uncertainties. Amplitude uncertainty is what most researchers typically think of as uncertainty. It refers to how well one knows the amplitude of the measurement. For example, if a measured temperature is stated as $700 \mathrm{~K} \pm 5 \mathrm{~K}$, the amplitude uncertainty is $5 \mathrm{~K}$. Unless otherwise stated, an uncertainty given in this paper is a standard uncertainty, which corresponds to a coverage factor of 1, or 1 standard deviation. ${ }^{(12)}$ This contrasts to expanded uncertainty, which corresponds to a coverage factor of 2 , or 2 standard deviations. Temporal uncertainty refers to how well one knows when a measurement occurred. If a measurement occurred $100 \mathrm{~ms} \pm 2 \mathrm{~ms}$ after time zero, the temporal uncertainty is $2 \mathrm{~ms}$. Spatial uncertainty refers to how well one knows the location in space of the measurement. If the position of a given pixel in an image corresponds to $100 \mu \mathrm{m}$ from the edge of a specimen $\pm 1 \mu \mathrm{m}$, the spatial uncertainty is $1 \mu \mathrm{m}$. 
To determine which uncertainty sources are of most concern, it is useful to first consider which have the greatest impact on the quantity being measured. For example, if one wished to know when a peak in the average temperature occurred, uncertainty sources which affect temporal uncertainty are probably of greater concern than those which effect amplitude or spatial uncertainty. However, one must remember that one uncertainty may also affect another uncertainty. For example, suppose one wished to know the temperature $200 \mu \mathrm{m}$ from an edge. If temperatures a short distance away have very different amplitudes (there are large thermal gradients in the image), even a modest spatial uncertainty can cause a large amplitude uncertainty, regardless of how well the sensitivity of the camera is known.

There are many other issues involved in uncertainty analysis which are described elsewhere. ${ }^{(7 ; 12)}$

\section{UNCERTAINTY SOURCES}

In this report, uncertainty sources are divided into five categories: calibration of camera sensitivity and offset, conversion of apparent to true temperature, camera optics, electronic effects, and other issues. Generalities for each category will be presented, along with specific illustrative examples.

\subsection{Calibration of camera sensitivity and offset}

The first category of uncertainty sources is related to the camera sensitivity (the slope of the line in a linear calibration curve) and offset (the intercept of the line). This category directly affects the amplitude uncertainty of the temperature measurement. Five examples from this category are discussed below.

\section{Example 1: Emissivity of the calibration source.}

When using a blackbody to calibrate a thermal camera, it is tempting to assume the emissivity of the blackbody is exactly 1 . However, consider the case of imaging two blackbodies, each set to $800{ }^{\circ} \mathrm{C}$, with a camera sensitive to a wavelength of $4 \mu \mathrm{m}$. The first blackbody has an apparent temperature of $784{ }^{\circ} \mathrm{C}$ while the second blackbody has an apparent temperature of $797^{\circ} \mathrm{C}$. According to the manufacturer, the first blackbody has an emissivity of 0.95 . This is typical for a plate blackbody. The second blackbody has an emissivity of 0.99 , which is typical for a spherical cavity blackbody. The $13{ }^{\circ} \mathrm{C}$ disagreement is due solely to the difference in emissivities. Not taking emissivity into account can lead to significant errors, even when emissivity is close to 1 . These quantities may be computed my manipulating Equation 4 or Equation 5 in Example 6. 


\section{Example 2: Use of an appropriate aperture.}

The light entering a lens is not restricted to the light emanating from within the field of view of the camera. Light from outside the field of view also enters the lens, and adds to the overall brightness level of the image due to reflections and scattering in the optical system. Shown in Figure 11, this may potentially effect both calibration and imaging of machining.

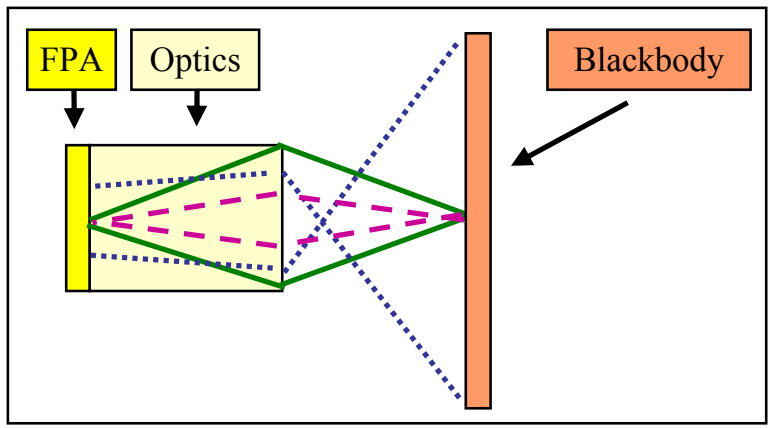

Due to stray light entering the lens, the blackbody appears brighter than it should. Also, the copious heat energy can significantly heat the lens.

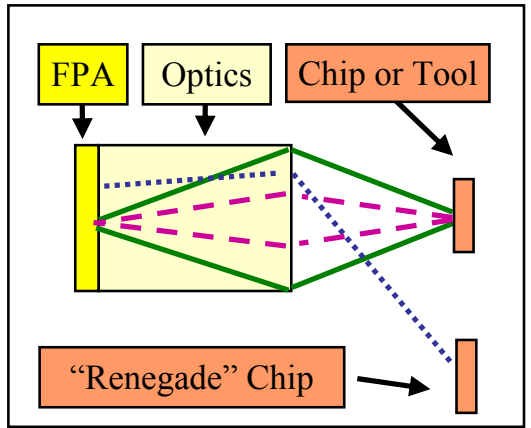

During machining, if a hot chip should travel to certain locations in space, stray light can enter the lens causing the image to appear brighter than it should.

Fig. 11. Effects of stray light.

An aperture is a hole or an opening through which light travels. Such holes may also be referred to as stops or aperture stops. For convenience, we will always use the word aperture. When calibrating, the effects of stray light may be minimized by adding an aperture. As shown in Figure 12, there are two ways an aperture may be used.

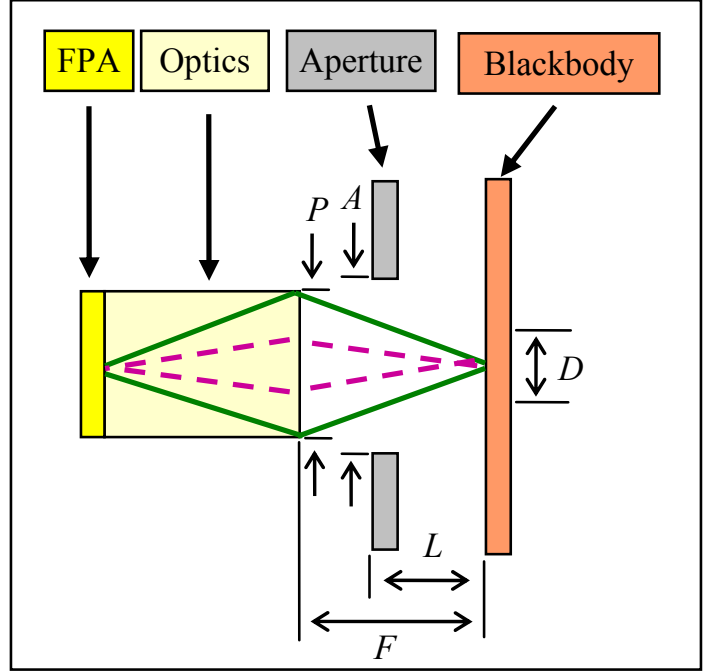

Focusing on the blackbody. $A \approx S(D+L P / F)$.

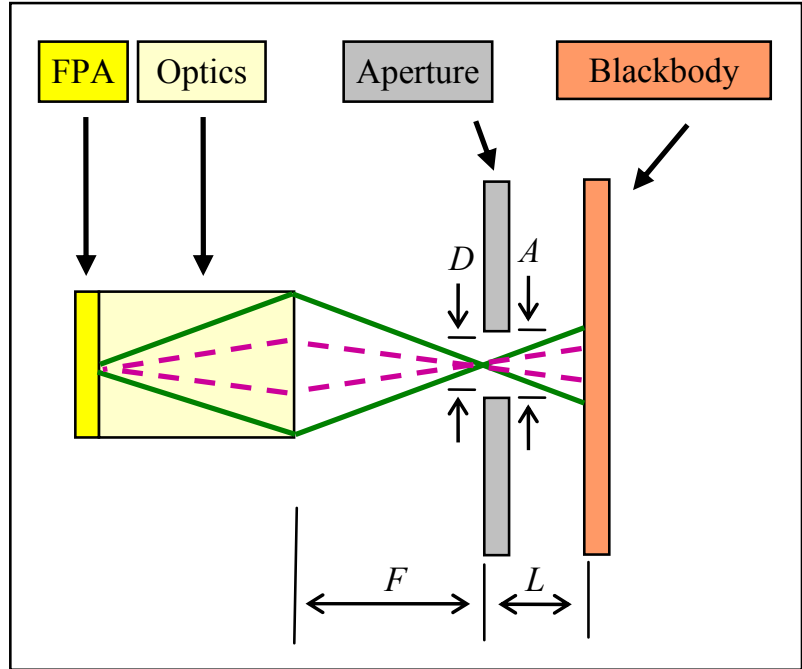

Focusing on the aperture. $A \approx S D$.

Fig. 12. Focusing on the blackbody requires a larger aperture than when the focus plane lies on the aperture. Thus, focusing on the aperture blocks more stray light. However, when focusing on the aperture, the size of the blackbody must be much larger than the aperture and the distance $L$ must be reasonably small. 
The MADMACS has approximately a $1 \mathrm{~mm}$ wide field of view. When calibrating, we focus in the plane of the $1.6 \mathrm{~mm}$ diameter aperture shown in Figure 13. Our calibration procedure is discussed more in Example 14.

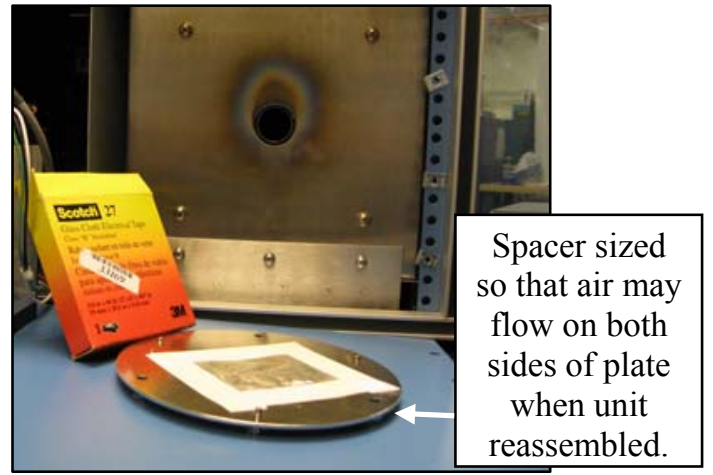

High-temperature glass tape is used to attach aluminum foil aperture to the metal disk.

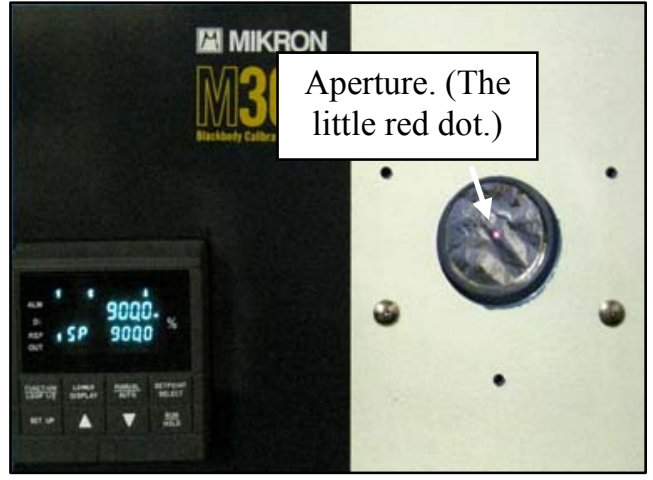

Reassembled blackbody ready for use.

Fig. 13. A method for mounting an aperture to the blackbody when the distance from the lens to the object being imaged is short.

Every optical system has a different sensitivity to stray light. By comparing intensity readings of the blackbody with and without an aperture, one may gauge how sensitive a particular system is to stray light. Our camera measures $10 \%$ higher intensity when no aperture is used. Table 1 shows the resulting deviation in the imaged temperature at various imaged temperatures. This table may be derived by manipulating Equation 4 or Equation 5 in Example 6.

\begin{tabular}{|c|c|}
\hline $\begin{array}{c}\text { Imaged Temperature } \\
\left({ }^{\circ} \mathrm{C}\right)\end{array}$ & $\begin{array}{c}\text { Deviation } \\
\left({ }^{\circ} \mathrm{C}\right)\end{array}$ \\
\hline 400 & +13.5 \\
\hline 500 & +17.6 \\
\hline 600 & +22.0 \\
\hline 700 & +27.0 \\
\hline 800 & +32.5 \\
\hline 900 & +38.1 \\
\hline 1000 & +44.2 \\
\hline
\end{tabular}

Table 1. Deviations at various imaged temperatures resulting from a $+10 \%$ increase in intensity due to stray light captured by the camera. 


\section{Example 3: Understanding resolution and repeatability}

Briefly stated, resolution is the smallest possible difference between two values of the measurand that can be measured and repeatability is the range of values attained by successive measurements under the same conditions. The intensity value at any pixel in an image is a digitized representation stored as binary Digital Levels, or DLs. Since intensity is acquired in DLs, one may argue that the best possible resolution is the intensity corresponding to the least significant bit of a DL value.

However, even if the response of a camera is a linear function of intensity, it is not a linear function of temperature, and thus resolution of the temperature measurement depends on the temperature being measured. Figure 14 illustrates this. If one wished to write specifications for a camera, one could specify that for a range of $10{ }^{\circ} \mathrm{C}$ to $290{ }^{\circ} \mathrm{C}$, the resolution is $0.6{ }^{\circ} \mathrm{C}$ or better, or that for a range of $100{ }^{\circ} \mathrm{C}$ to $290{ }^{\circ} \mathrm{C}$, the resolution is $0.1{ }^{\circ} \mathrm{C}$ or better. While the two specifications seem very different, they actually describe the same camera. Thus, any comparison of cameras or camera settings must be over the same temperature range. Also note that within the same thermal image, hotter portions have a finer temperature resolution than colder portions. A similar argument may be applied to repeatability.

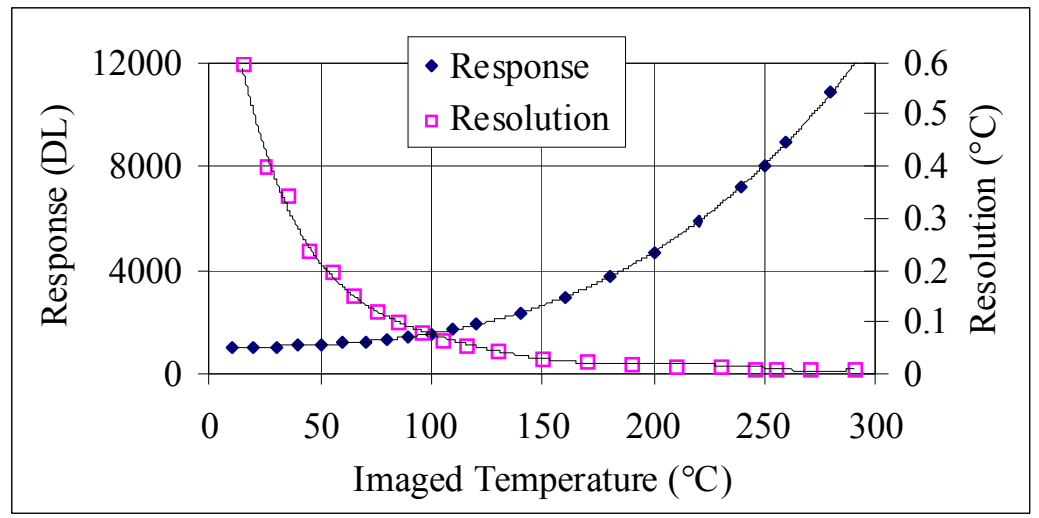

Fig. 14. Response of a camera in DLs, and resulting resolution, as a function of temperature. 


\section{Example 4: The range of linear operation}

In general, each pixel on a FPA has an "S" shaped response curve. The size and shape of the curve may be manipulated by adjusting the integration time or the optical filtering used. Figure 15a shows a situation with two desirable characteristics. First, only the linear range of the camera is used. Second, the entire linear range is used, not a small section of it. Figure 15b shows just the lower portion of the same curve.

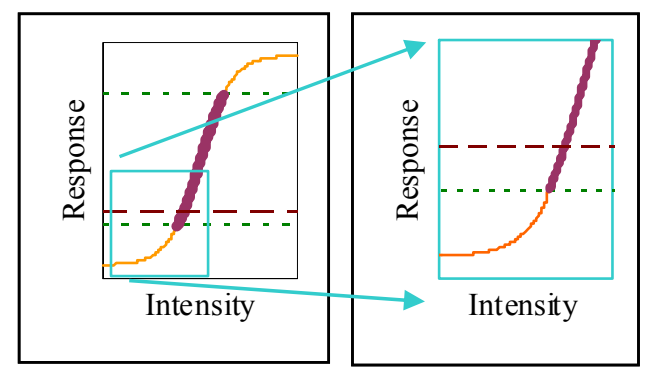

$\mathrm{a}$

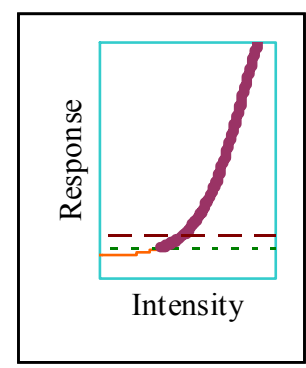

$\mathrm{c}$

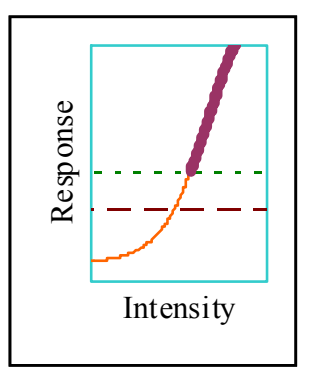

d

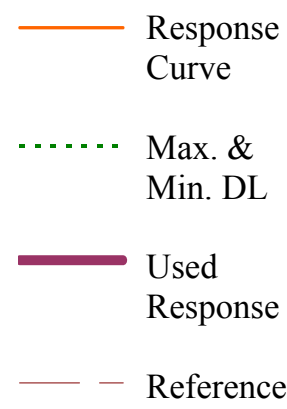

Plate

Fig. 15. Idealized camera response. $15 \mathrm{a}$ and $15 \mathrm{~b}$ show an ideal situation while $15 \mathrm{c}$ and $15 \mathrm{~d}$ are problematic.

A formula describing the intensity response of the camera system is

$$
C=E_{\mathrm{e}}+R\left(E_{\mathrm{o}}+I\right)
$$

A single point calibration of a thermal image is when a single value is subtracted from each pixel in the image. A single point calibration only corrects for errors in the offsets of the camera response at each pixel. This contrasts with a two point calibration, which uses a cold image and a hot image to correct both offset and slope errors. To remove both optically and electronically induced offsets in $C$, it is common practice to use a room temperature "reference plate" to perform a single point calibration. If the camera is used only in the linear portion of the response, then

$$
R\left(E_{\mathrm{o}}+I\right)=R\left(E_{\mathrm{o}}\right)+R(I)
$$

Equation 1 may be restated as

$$
C=E_{\mathrm{e}}+R\left(E_{\mathrm{o}}\right)+R(I)=E_{\mathrm{c}}+R(I) .
$$

Subtracting a constant, such as a reference plate reading, works as a single point calibration in this case.

As shown in Figure 15c, a researcher may wish to extend the dynamic range of the camera by deliberately using the camera beyond the linear range. However, as a result, Equation 2 and Equation 3 might not apply and errors could result. This does not mean that the dynamic range of the measurement can not be extended 
by using both the linear and nonlinear portions of the response. It does mean that methodologies used when converting FPA readings to temperature need reevaluation.

Figure $15 \mathrm{~d}$ shows another potentially problematic situation. Here, the camera is operated within the linear range, but the reference plate is at too low a temperature (intensity) and the measured digital reading is 0 (zero) DLs. All objects emit light due to their temperature - even the optics of the camera system. Imagine a scenario where the temperature of the optics increases or decreases, causing them to emit more or less light. This causes the intensity of light received by the FPA while measuring the reference plate to change. However, since the reference plate reading is below zero DLs, the camera only reports zero and thus the change is not compensated for. This error may be avoided by using a reference plate at a high enough temperature that the camera yields DLs above zero.

As discussed in Example 14, each pixel in the FPA has a different response. Thus, it is possible for some pixels to be operating in the linear range (Figure 15b) while others are not (Figure 15c for example). When gauging whether the FPA is operating in the linear range, one can not consider just an average response, but the responses of the individual pixels as well.

\section{Example 5: Sensitivity to polarization}

Another source of uncertainty is the dependency of camera sensitivity to polarization of the measured thermal radiation. For polarization to be a significant source of uncertainty, the thermal radiation measured must be at least partially polarized and the sensitivity of the camera system must vary as a function of polarization angle.

To estimate uncertainty, we will only consider linear polarization and ignore circular polarization. That is, we will assume that $S_{3}$ of the Stokes vector ${ }^{(13)}$ is 0 . The sensitivity of MADMACS to polarization was estimated by imaging a blackbody through a linear polarizer rotated at various angles. For MADMACS, there is approximately a $\pm 5 \%$ variation in intensity. Table 2 shows deviations which result from a $\pm 5 \%$ variation in intensity at various imaged temperatures. This table may be derived by manipulating Equation 4 or Equation 5 in Example 6.

\begin{tabular}{|c|c|c|}
\hline $\begin{array}{c}\text { Imaged } \\
\text { temperature } \\
\left({ }^{\circ} \mathrm{C}\right)\end{array}$ & $\begin{array}{c}\text { Deviation when all light is linearly } \\
\text { polarized in the sensitive direction } \\
\left({ }^{\circ} \mathrm{C}\right)\end{array}$ & $\begin{array}{c}\text { Deviation when all light is linearly polarized } \\
\text { perpendicular to the sensitive direction } \\
\left({ }^{\circ} \mathrm{C}\right)\end{array}$ \\
\hline 400 & +6.5 & -7.0 \\
\hline 500 & +8.6 & -9.0 \\
\hline 600 & +10.9 & -11.1 \\
\hline 700 & +13.3 & -13.7 \\
\hline 800 & +16.1 & -16.4 \\
\hline 900 & +18.9 & -19.2 \\
\hline 1000 & +21.9 & -22.3 \\
\hline
\end{tabular}

Table 2. Deviation at various imaged temperatures resulting from a $\pm 5 \%$ variation in intensity.

There are a variety of reasons to suspect that the light observed during machining might be partially polarized. One is that when a surface is viewed from an angle not perpendicular to the surface, both reflected and emitted light may be polarized ${ }^{(6 ; 14 ; 15)}$. In fact, a polarizer may be used to reduce the effect of reflections in thermal imaging ${ }^{(14)}$. Ongoing research involves imaging the machining process through a rotating linear polarizer to determine if there is a significant amount of polarization. 


\subsection{Conversion of apparent to true temperature}

Once a thermal image is acquired and converted to apparent temperatures, the next step is to convert apparent to true temperatures. The conversion of apparent to true temperature is the second category of uncertainty sources considered. This category directly affects the amplitude uncertainty of the temperature measurement. While uncertainties in emissivity generally dominate, other issues may be important as well. Three examples are discussed next.

Example 6: The form of the equation used to convert to true temperature.

Planck's equation describes the radiance of a black body at a certain wavelength as a function of the true temperature:

$$
I=\frac{c_{1 \mathrm{~L}}}{\lambda^{5} \cdot\left(e^{\frac{c_{2}}{\lambda \cdot T_{\text {true }}}}-1\right)} .
$$

There are multiple modifications of Planck's equation in the literature. Some take into account effects such as reflectivity, transmittance, atmospheric absorption, multiple reflections, changes in emissivity as a function of temperature ${ }^{(16)}$, or whether to use a single wavelength or integrate over a range of wavelengths. We use the form

$$
M=\|\left[\int_{\lambda_{1}}^{\lambda_{2}} \frac{w_{\lambda}}{\lambda^{5} \cdot\left(e^{\lambda \cdot T_{\text {apparent }}}-1\right)} d \lambda\right]-\left[\varepsilon \cdot \int_{\lambda_{1}}^{\lambda_{2}} \frac{w_{\lambda}}{\lambda^{5} \cdot\left(\frac{c_{2}}{e^{\lambda \cdot T_{\text {true }}}}-1\right)} d \lambda\right]-\left[(1-\varepsilon) \cdot \int_{\lambda_{1}}^{\lambda_{2}} \frac{w_{\lambda}}{\lambda^{5} \cdot\left(\frac{c_{2}}{\lambda \cdot T_{\text {environment }}}-1\right)} d \lambda \| .\right.
$$

This form takes the spectral response of the camera $\left(w_{\lambda}\right)$, emissivity $(\varepsilon)$, and reflection of the environmental temperature into account. An unknown quantity may be determined by substituting known values and adjusting the unknown value until $M$ is minimized. This works well for determining $T_{\text {apparent }}, T_{\text {true }}$, or $\varepsilon$. Due to the fact that the temperature of the environment (and thus the brightness) is generally significantly lower than the temperature of the hot chips, solving for $T_{\text {environment }}$ often has a large uncertainty. This equation may also be used to produce the nomograms ${ }^{(7)}$ shown in Appendix II. A nomogram is a graph designed to allow the approximate computation of a function. It may also be used to perform an approximate uncertainty analysis.

Most researchers use a filter to ensure that a single, well known wavelength is detected by the camera. However, this significantly reduces the sensitivity of the camera, which necessitates the use of longer integration times, causing significant motion blur and washing out most of the detail in the thermal images. As will be discussed in Example 7, we do not use such filters and are thus able to have an integration time of only $10 \mu \mathrm{s}$. This limits the motion blur to only a few pixels, preserving most of the detail. However, to accurately convert apparent to true temperature, the camera sensitivity as a function of wavelength must be known. 
Table 3 shows an example of how computed true temperature is affected by the assumptions made for MADMACS. While the difference in this case is only about $6^{\circ} \mathrm{C}$, this is a significant difference given that it was due solely to improper execution of the conversion procedure.

\begin{tabular}{|l|c|c|c|}
\hline \multicolumn{1}{|c|}{ Assumption about camera response as a function of $\lambda$} & $\begin{array}{c}\lambda_{1} \\
(\mu \mathrm{m})\end{array}$ & $\begin{array}{c}\lambda_{2} \\
(\mu \mathrm{m})\end{array}$ & $\begin{array}{c}\text { Computed } T_{\text {true }} \\
\left({ }^{\circ} \mathrm{C}\right)\end{array}$ \\
\hline Measured response of system (constant between $\lambda_{1}$ and $\left.\lambda_{2}.\right)$ & 3.8 & 5.1 & 1086 \\
\hline Single measured (average of high and low measured response of system) & 4.45 & 4.45 & 1092 \\
\hline Single "nominal" (see text) & 4.35 & 4.35 & 1086 \\
\hline
\end{tabular}

Table 3 Various values of $T_{\text {true }}$ resulting from different assumptions about $\lambda$, where $T_{\text {apparent }}=800{ }^{\circ} \mathrm{C}, T_{\text {environment }}=20^{\circ} \mathrm{C}$, $\varepsilon=0.5$, and $w_{\lambda}=1$ for all $\lambda$.

While using a single, empirically derived "nominal" wavelength value ( $4.35 \mu \mathrm{m}$ in this case) is a good approximation to using the entire measured response $(3.8 \mu \mathrm{m}$ to $5.1 \mu \mathrm{m}$ in this case), notice that the "nominal" value is not the arithmetic mean of the low and high limits for the wavelength. Also, this approximation is only good over the temperature range of about $50{ }^{\circ} \mathrm{C}$ to $1500{ }^{\circ} \mathrm{C}$. If one measures temperatures well outside the range of normal machining temperatures, this approximation becomes inaccurate. In addition, if one is using a thermal camera sensitive to a very wide range of wavelengths (e. g., $1 \mu \mathrm{m}$ to $5 \mu \mathrm{m}$ ), this type of approximation can have large errors even over the range of temperatures found in machining.

\section{Example 7: Nonuniform surface textures.}

Surface texture has a very significant impact on the emissivity of an object. As metal is machined, it is severely deformed. The surface texture of chips is much rougher and nonuniform than the surface of the workpiece. Thus, handbook values for emissivity of the chips are not useful and must be measured. As will be shown, the large variability in surface texture means that there is a large variability in emissivity across the chip. To measure the emissivity of the side of the chips imaged during machining, two chips are placed in a pin vise with a thermocouple sandwiched between them. Shown in Figure 16, thermal tweezers are then used to heat the chips to a known temperature. The chips are imaged by the dual spectrum system and the difference between the thermocouple and apparent temperature is attributed to local emissivity, $\varepsilon$, of the chip surface. Due to the extreme deformation involved, chips typically have a much rougher surface than the workpiece. One expects the chips to be more emissive than the workpiece due to this dramatic increase in surface roughness.

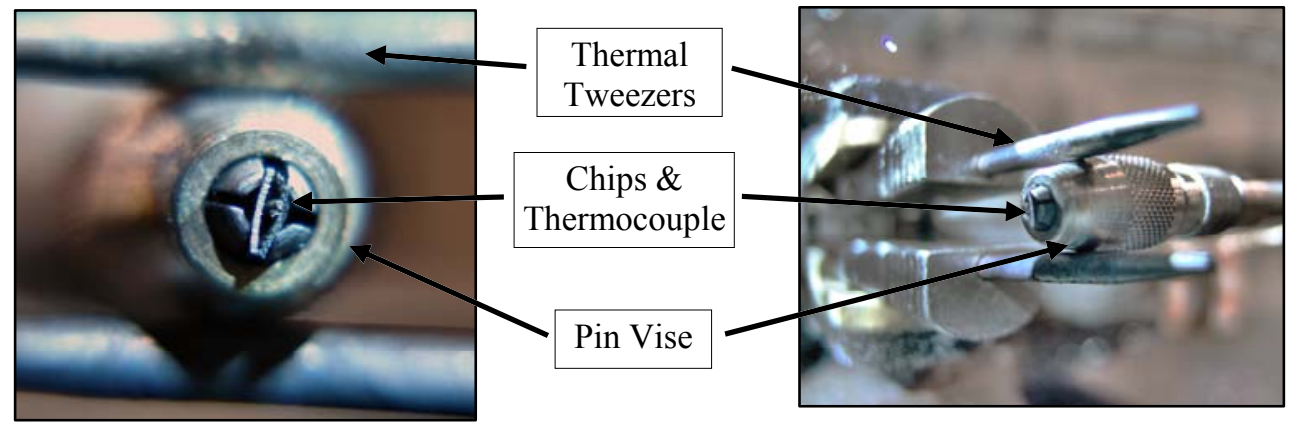

Fig. 16. Two steel chips are heated to a known temperature to estimate their emissivity. 
Figure 17 shows a visible and thermal spectrum image of a segmented chip heated as shown in Figure 16 . The side flow is often out of focus due to the limited depth of field of the camera system. Emissivity $\varepsilon \approx 0.3$ for the segment faces while $\varepsilon \approx 0.5$ for the gap and side flow portions of the image. It is not uncommon for emissivity values to vary from 0.2 to 0.6 on a single segment face. With such large variation in emissivity, a large temperature uncertainty is difficult to avoid.

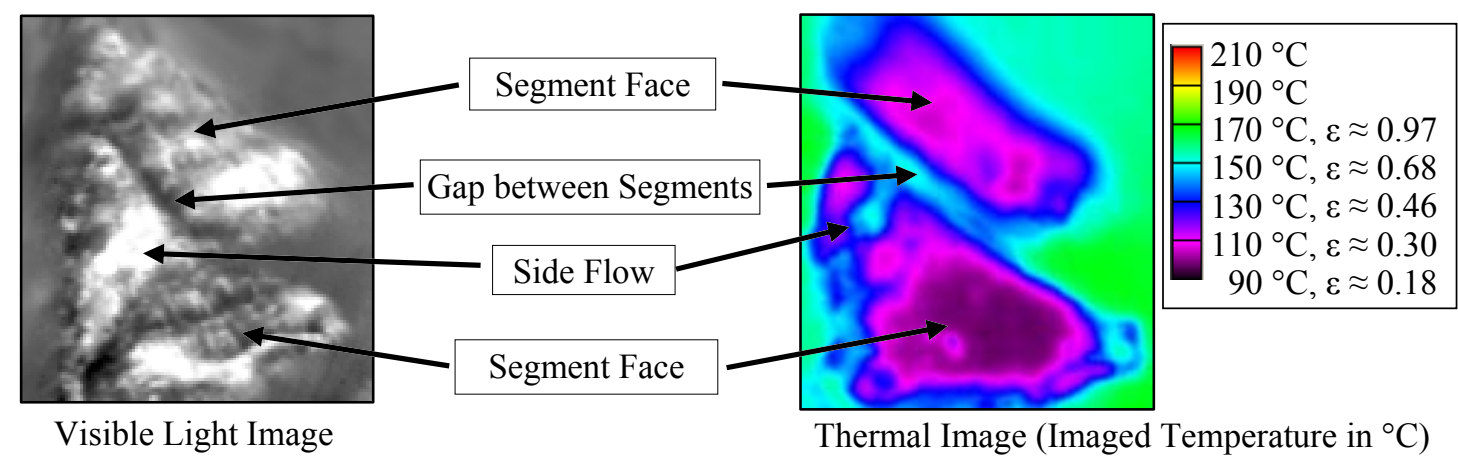

Fig. 17. A visible and thermal spectrum image of imaged temperatures with approximate corresponding emissivity values. Imaged temperatures are assumed to be approximately equal to apparent temperatures. The $172{ }^{\circ} \mathrm{C}$ chip is about $0.6 \mathrm{~mm}$ thick (left to right in the image).

Figure 18 shows a gap between segments during machining. It appears to be much hotter than the chip itself. However, the gap may have formed a micro-blackbody, raising the effective emissivity. The lowest imaged temperature detectable at these camera settings is $230{ }^{\circ} \mathrm{C}$. Assuming imaged temperatures are approximately equal to apparent temperatures, and using an emissivity value of 0.3 (from Figure 17), the hottest true temperature possible for the segment faces is about $345^{\circ} \mathrm{C}$. Using an emissivity value of 0.5 for the gap (also from Figure 17), the true temperature of the gap is somewhere between $370{ }^{\circ} \mathrm{C}$ and $510{ }^{\circ} \mathrm{C}$. If one had assumed an emissivity of 0.3 for the gap, a true temperature between $440{ }^{\circ} \mathrm{C}$ and $620{ }^{\circ} \mathrm{C}$ would have been computed instead. Thus, the gap is actually hotter than the face, but not by as much as one would have predicted if the nonuniform emissivity was not correctly accounted for. There are other sources of uncertainty effecting this measurement that will be discussed in Example 10 and Example 19.

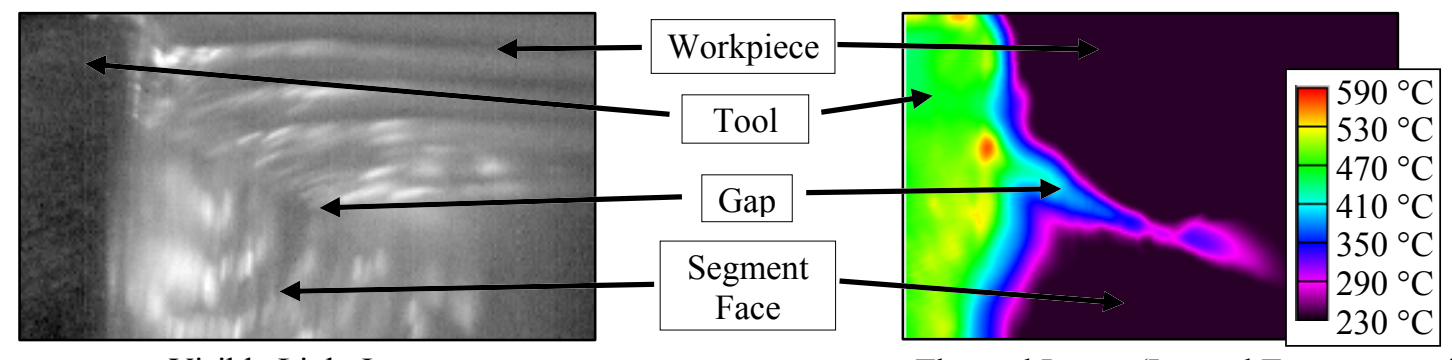

Visible Light Image

Thermal Image (Imaged Temperature in ${ }^{\circ} \mathrm{C}$ )

Fig. 18. Visible and thermal spectrum images of segmented chip formation.

Some researchers attempt to avoid the issue of nonuniformity by using a long integration time while imaging chip formation. The motion of the chip causes each pixel of the resulting images to be an average of many locations on the chip surface. It is then claimed that this yields an average temperature. However, while each pixel may represent an average intensity, it does not yield an average (arithmetic mean) temperature. This is 
because the equation converting intensity to temperature is highly nonlinear. Uncertainty due to integration time and surface variability is largest when the integration time is long and the surface is highly nonuniform.

During a long integration time, chip motion causes different portions of the rapidly moving chips to be projected onto any given pixel. When the chips are segmented, there are two types of portions, faces of the chips and gaps between the segments. Assuming a perfect camera, each portion has an instantaneous intensity which is determined by the true temperature and emissivity of that portion. At the end of the integration time, the average of these instantaneous intensities is what the camera has measured for that pixel and reports in DLs. Figure 19 shows results of a calculation (Appendix 3) to determine the deviation between the measured true temperature and the actual true temperature which results when one images segmented chip formation using a long integration time. One may think of the value reported for a pixel as the result of a population of [ $\left.T_{\text {true }}, \varepsilon\right]$ pairs $\left(T_{\mathrm{j}}\right.$ and $\varepsilon_{\mathrm{k}}$ in Appendix 3). For Figure 19, it is assumed that the portions representing the chip faces constitute about $90 \%$ of the total population, and are clustered around $\left[T_{\text {true }}=600{ }^{\circ} \mathrm{C}, \varepsilon=0.3\right]$ and that the gaps constitute about $10 \%$ of the total population, and are clustered around $\left[T_{\text {true }}=800{ }^{\circ} \mathrm{C}, \varepsilon=0.7\right]$. The probability that a pixel is exposed to a given $\left[T_{\text {true }}, \varepsilon\right]$ pair $\left(N_{\mathrm{j}, \mathrm{k}}\right.$ in Appendix 3$)$ at any instantaneous time is shown on the left of Figure 19. The resulting intensity the pixel is exposed to for each $\left[T_{\text {true, }}, \varepsilon\right]$ pair $\left(I_{\mathrm{j}, \mathrm{k}}\right.$ in Appendix 3) is shown on the right. By integrating these curves, one may compute the actual average true temperature ( $a T$ in Appendix 3) and true temperature the camera reports ( $p T$ in Appendix 3). In this case, the difference between the actual and measured true temperature is about $27^{\circ} \mathrm{C}$. The reason is that, while the gaps are only about $10 \%$ of the population, they contribute almost $50 \%$ of the intensity, thus dominating the average intensity. Similar analyses on populations representing typical continuous (not segmented) chips indicate that this deviation is much smaller.

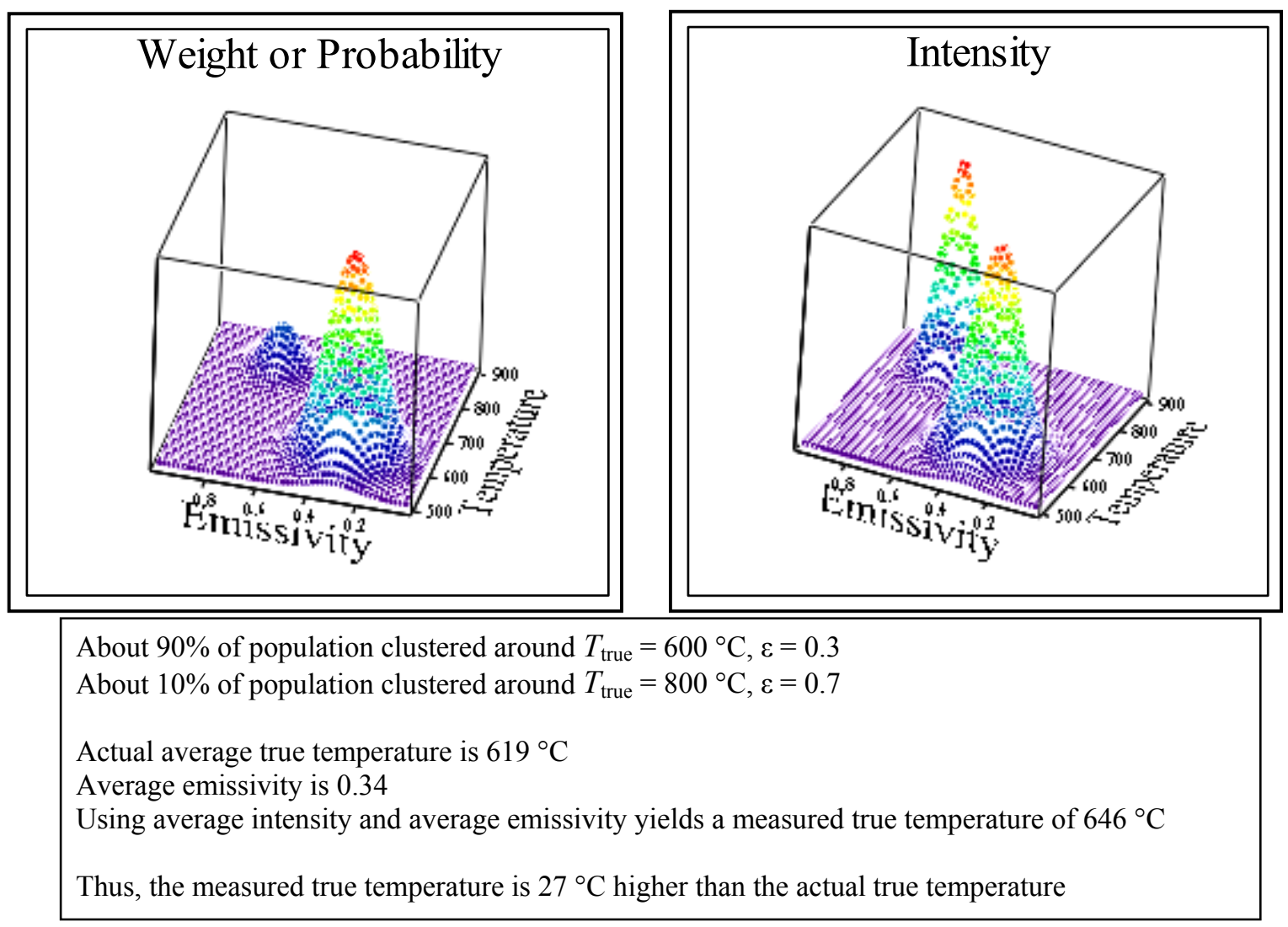

Fig. 19. An analysis of the effect of long integration time on average temperature when measuring segmented chips. 
It should be noted that even when only the faces of the segments are considered, the variation in emissivity is still significant. Figure 20 shows a histogram of emissivity data for just the faces of several adjacent segments from the same test. The average emissivity is 0.345 , with an expanded uncertainty of $0.05(k=2)$. This corresponds to almost $15 \%(k=2)$ uncertainty in the average emissivity value.

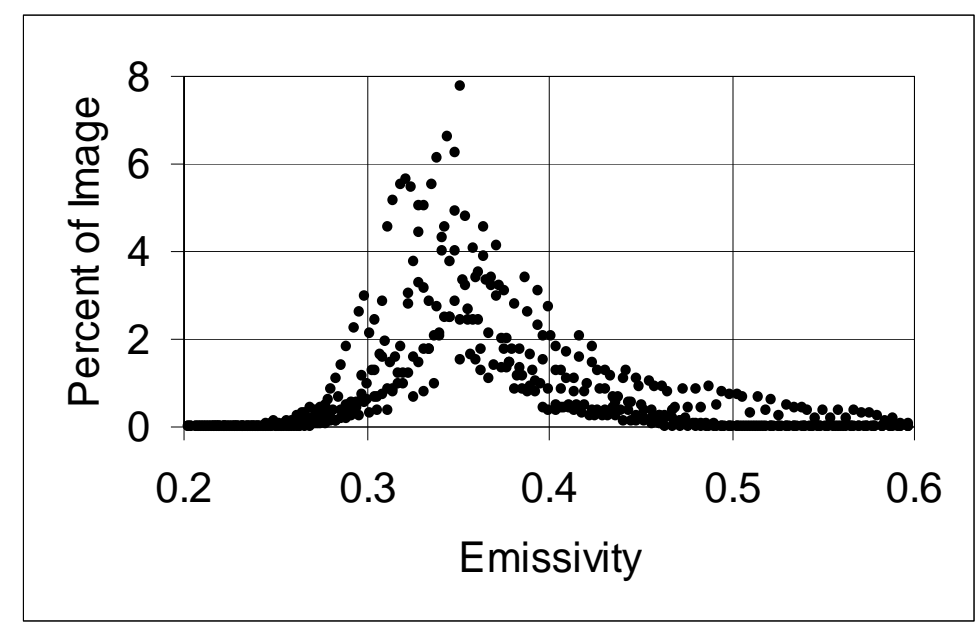

Fig. 20. Even when only the faces of the segments are considered, the variation in emissivity is still significant.

\section{Example 8: Oxide layer changes}

Figure 21 shows two chips from the same test. Note that, despite being from the same test, there is a significant color difference due to the difference in oxide layers. The top chip has an average emissivity of 0.4 , compared to 0.8 for the chip at the bottom. It is not obvious which value should be used to convert apparent to true temperature. If the oxide layer took time to form and did not form until after the chip had left the field of view of the thermal camera, perhaps neither is correct!

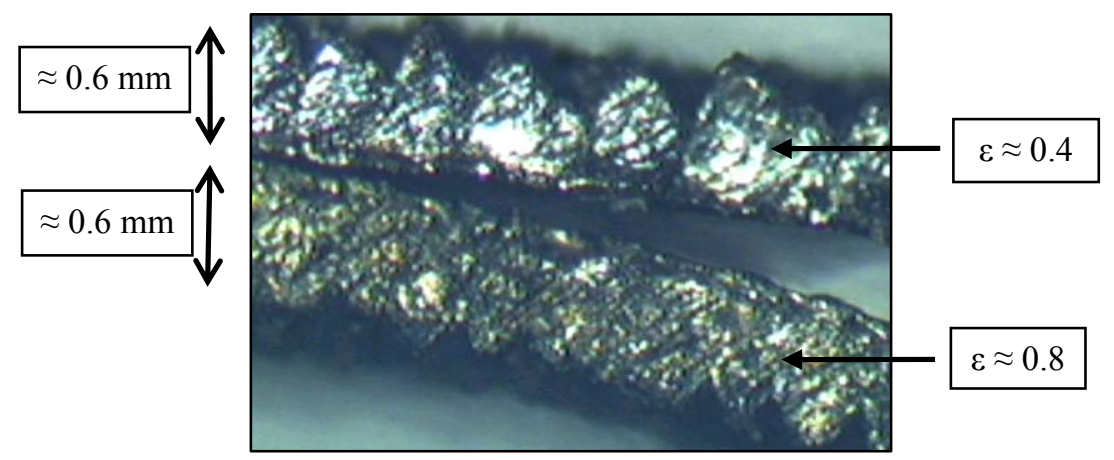

Fig. 21. Two chips about $0.6 \mathrm{~mm}$ thick (vertical in the image) from the same cutting test, but with different oxide layers. 
Figure 22 illustrates that oxidation is a reaction that takes time to occur. The video is available on the NIST website $^{(9)}$. In this case, oxidation formation is an exothermic (produces heat) process. These two images, acquired from two different vantage points at the same moment in time, show a very thin chip that, as it emerges from the tool, is not hot enough to visibly glow. Further down the chip, several milliseconds have past since this portion of the chip has been cut, yet it is hot enough to glow very brightly. The once solid chip sometimes melts and boils well after the test is finished. The reason the temperature rise is not obvious with normal, thicker chips is that thick chips have a lot more metal to absorb the exothermic heat energy. That is, thick chips have a higher volume to surface area ratio. It should be noted that oxidation is not always exothermic. Other workpiece materials may also be either endothermic (receives heat from the surroundings) or temperature neutral.

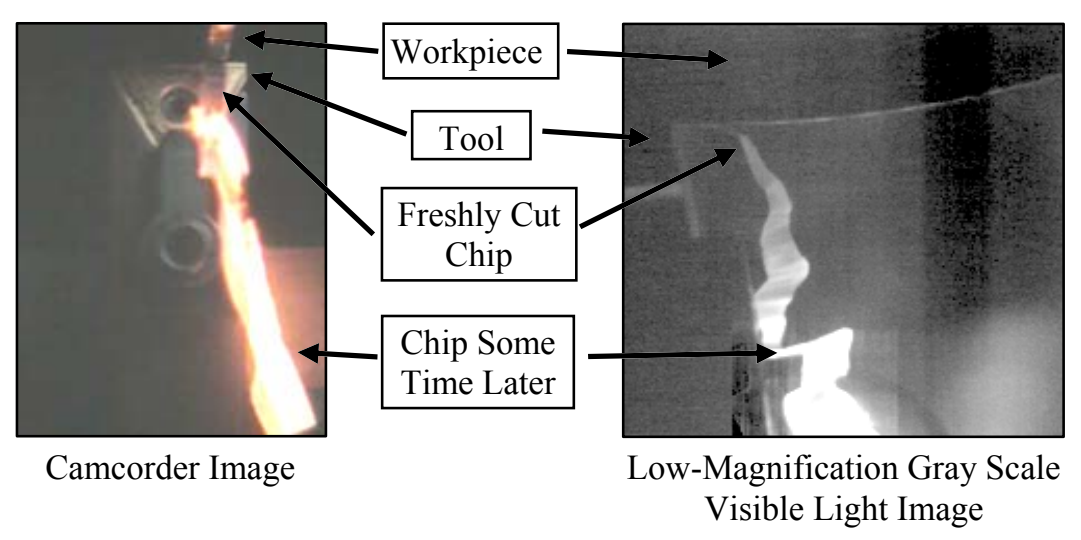

Fig. 22. A very thin chip, less than $0.1 \mathrm{~mm}$ thick, illustrating exothermic oxide formation.

There may also be some superheating of the material during cutting (being heated above the melting temperature but not having time to transform from a solid to a liquid). However, well after the chips have left the cutting zone, they glow dramatically brighter than what may be attributed to any emissivity changes alone. Thus, it is likely that an exothermic reaction is the dominant cause for this behavior.

\subsection{Camera optics}

No optical system yields perfect images under all conditions. An image may be distorted, or portions may be out of focus (as when there is extreme side flow). The optical system directly affects both amplitude and spatial uncertainty of the temperature measurements. Four examples will be discussed next. 


\section{Example 9: Optical pixel crosstalk}

Especially when there is a high ratio between the brightest and darkest areas of interest, unwanted scattering and reflections in the imaging system cause intensity values of some pixels to apparently affect intensity values of other pixels. Figure 23 shows a simple experiment designed to gauge this crosstalk. The thermal camera images a chilled, $500 \mu \mathrm{m}$ wide aperture. On the far side of the aperture, a removable foil blocks a hot blackbody. The camera acquisition is started with as high a frame rate as is practical. The foil is then rapidly removed. The thermal movie is examined, and a frame just before the foil is removed is compared to a frame just after the foil is removed.
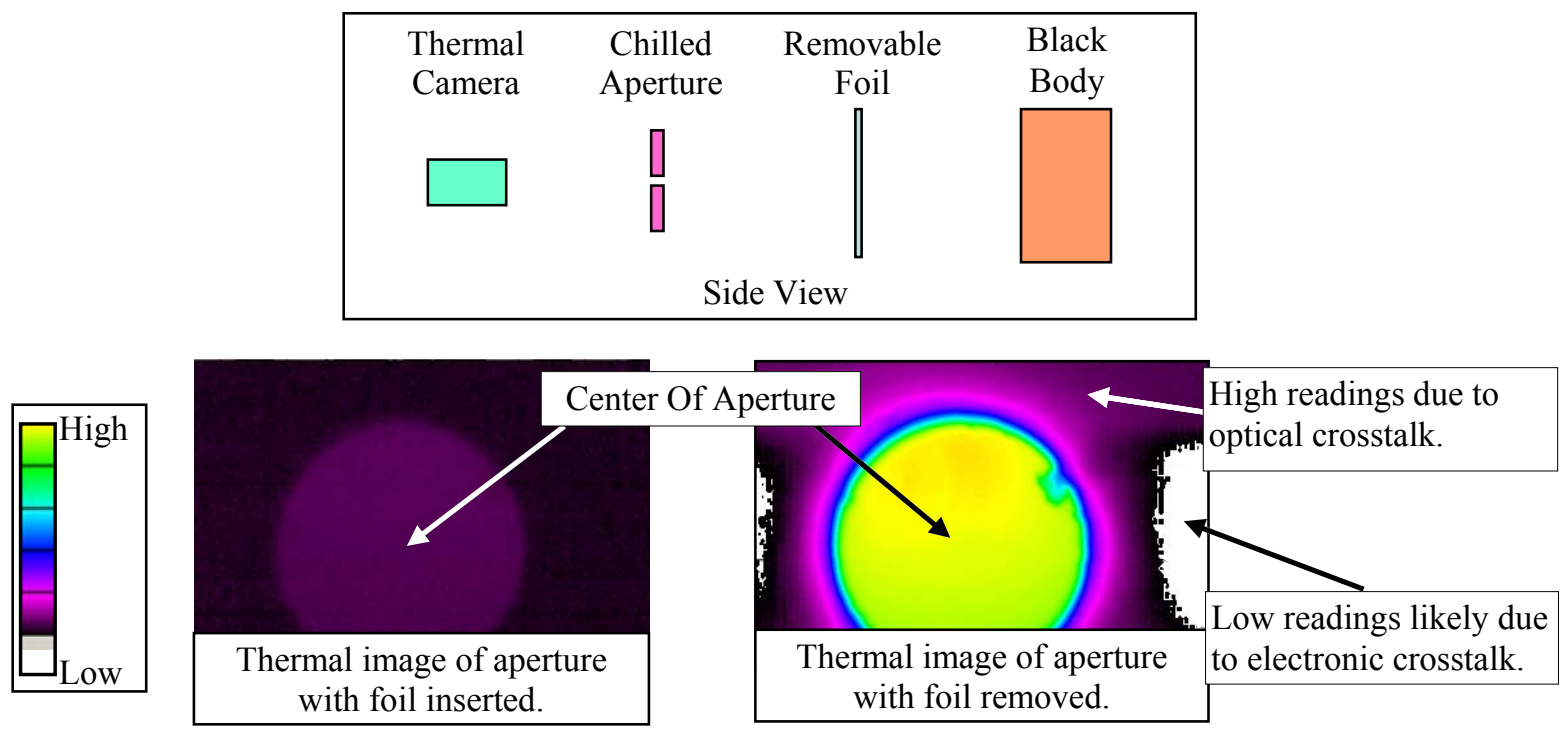

Fig. 23. A simple experiment used to gauge optical and electronic pixel crosstalk. Two images are compared, one with and one without the foil (which acts like a shutter). Electronic crosstalk is discussed in the next section.

The reason for the rapid foil removal and high frame rate is to minimize the amount of time between foil removal and acquisition of the second image. This reduces the possibility that the actual aperture temperature is affected by radiative heating from the blackbody.

As expected, the temperature in the center of the aperture is very high after the foil is removed. However, the region around the aperture also shows higher temperature due to scatter in the optical system. Notice that in the center of the left and right sides of the image, the imaged temperature has actually decreased. While this theoretically could be caused by the optical transfer function (OTF) of the system going negative ${ }^{(15)}$, it is more likely due to electronic pixel crosstalk, which is discussed in the next section. The OTF is the system response plotted as a function of spatial frequency. This contrasts to the point spread function, where the system response is plotted as a function of position.

\section{Example 10: Optical size-of-source effects}

The infrared light emitted by a very small object at a given temperature appears less bright than it does when the size of the object is above some threshold. This is the result of several effects, such as the PSF of the optical system, as well as whether the light from the object only partially fills a pixel on the FPA. This can be a significant source of uncertainty due to the small sizes of many features, for example the shear zone. 
There are various techniques for characterizing size-of-source effects. One is to measure the PSF of the camera. If the PSF is wide, the PSF is probably the dominant effect. If the PSF is narrow, effects such as only partially filling pixels on the FPA probably dominate. Measuring the PSF may be accomplished by using either a knife edge or circular edge technique ${ }^{(17)}$, where the transition from a light area to a dark area in the image is analyzed.

Another technique, shown in Figure 24, is to image a resolution target which has features of various sizes $^{(18 ; 19)}$. An aperture is used to limit how much the resolution target is heated by the blackbody. When properly back-lit by the black-body, the features on the resolution target are all equally bright. However, due to size-of-source effects, the camera reports the small features as less bright than the large ones.

The size-of-source effects for MADMACS are dominated by the PSF of the optics. This information is used to compensate for these effects in Example 19.

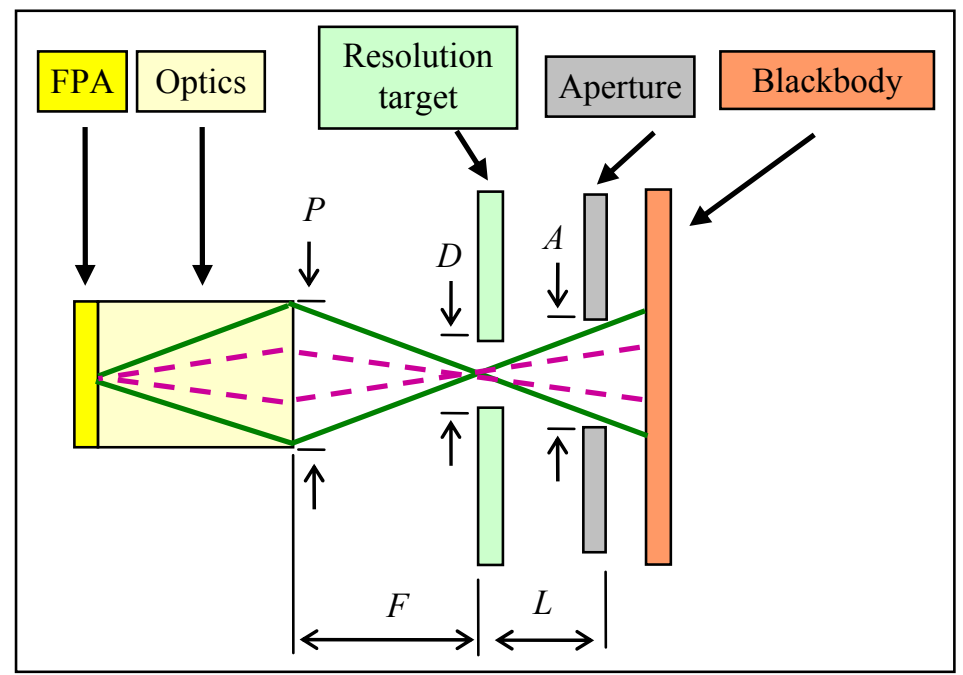

An aperture is needed to keep the resolution target from overheating. To insure the entire field of view is usable, the aperture must be larger than the $1.6 \mathrm{~mm}$ diameter aperture used in Example 2. $A \approx S(D+L P / F)$.

Fig. 24. Using a resolution target to characterize size-of-source effects.

\section{Example 11: Focus}

Any camera system has a plane, some distance $F$ in front of the lens, which yields the best quality image. However, there is some distance on either side of that plane which still yields an acceptable image, called the depth of focus. If an object lies outside of the depth of focus, it will appear both blurry and reduced in intensity in the image. 
Figure 25 illustrates how depth of focus can impact thermal images. The tool almost disappears when sufficiently out of focus. The same can occur for the chip. This makes positioning the camera challenging since the width of the chip depends on the cutting conditions and the material being machined.

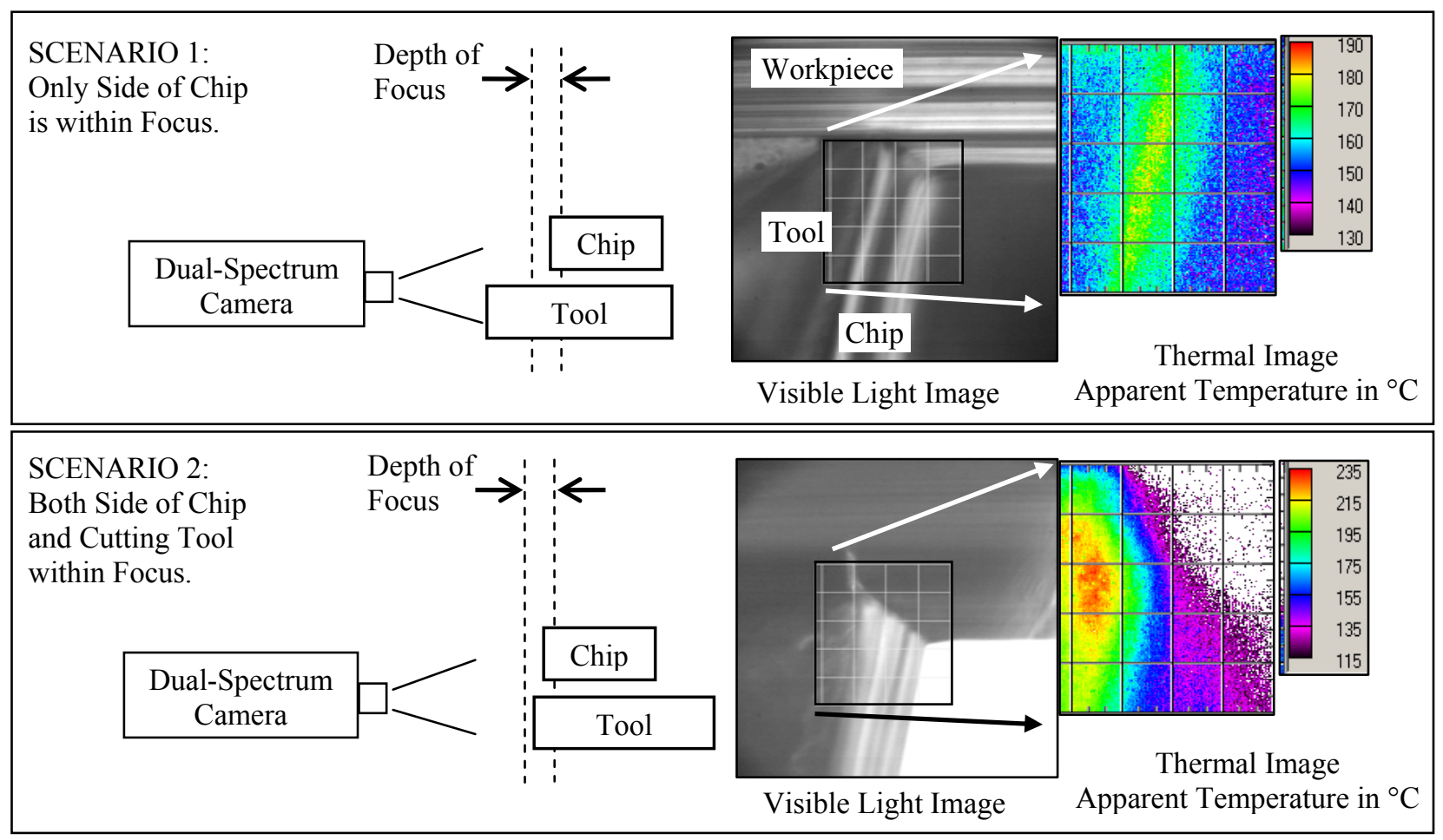

Fig. 25. Depth of focus affects what is imaged. The width of the field of view is $520 \mu \mathrm{m}$ for the visible light and $240 \mu \mathrm{m}$ for the thermal images.

\section{Example 12: Distortion}

One way to gauge the amount of distortion in a camera system is to image a target with regularly spaced features and look for deviations from the expected locations and shapes of the features. Generally, features become more distorted the further away they are from the center of the image. Figure 26 shows that, for this field of view, our system exhibits some distortion of the thermal images near the edges. We currently address this by making sure measurements are not made in the corners of the thermal images.

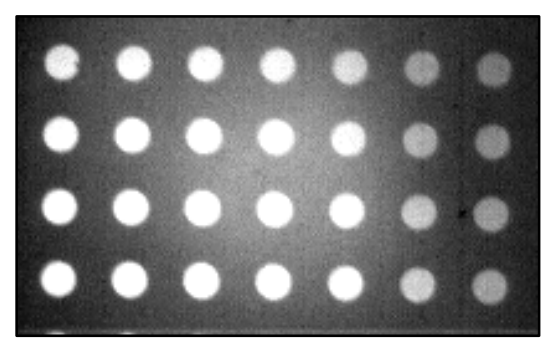

Visible Light Image

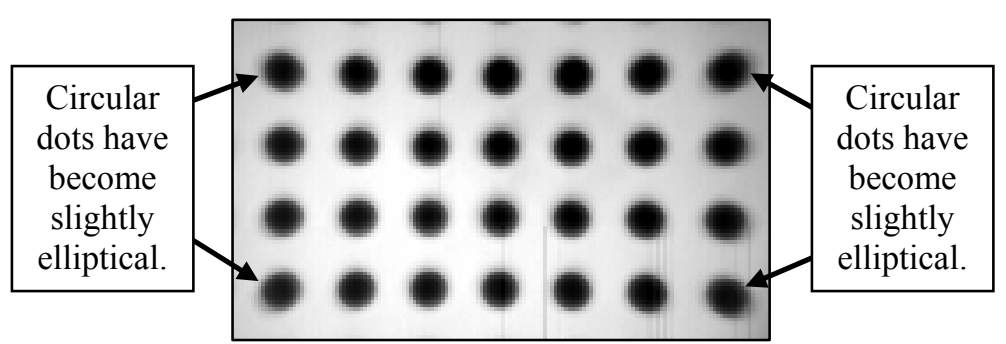

Thermal Image

Fig. 26. Imaging a target with $0.125 \mathrm{~mm}$ dot spacing to check image distortion. 


\subsection{Electronic effects}

A variety of uncertainty sources are associated with the electronics within the camera. They directly affect amplitude and temporal uncertainty in temperature measurements. Four examples are described below.

\section{Example 13: Electronic pixel cross talk}

A FPA where each pixel has its own power supply lines and analog-to-digital converter is simply not practical. Thus, pixel elements within a camera share electronic components. Figure 27 shows a simplified, stylized eight-pixel FPA. Any power supply line has some resistance. The power supply lines on sensor array chips are very thin, and thus the resistance is high when compared to normal copper wire. If the current is low, the voltage drop across the supply line is negligible. However, if a pixel element is exposed to a very bright intensity, it may draw more current than normal. This could cause a drop in the voltage supplied to other pixels, affecting their reading. In our eight-pixel sensor, an overdriven pixel $\mathrm{P}_{1}$ could affect pixel $\mathrm{P}_{2}$. The end result is that a few very bright pixels in an image may induce large errors in the dimmer ones. A similar situation arises from the fact that groups of pixels share a multiplexer and an analog-to-digital converter.

If pixel $\mathrm{P}_{1}$ is severely overdriven, or saturated, it often takes some time before it behaves normally again. This is sometimes referred to as the saturation recovery time. Thus, after a very bright object has passed through the field of view, affected pixels may appear brighter than they should for several frames afterwards. This is illustrated in Example 16.

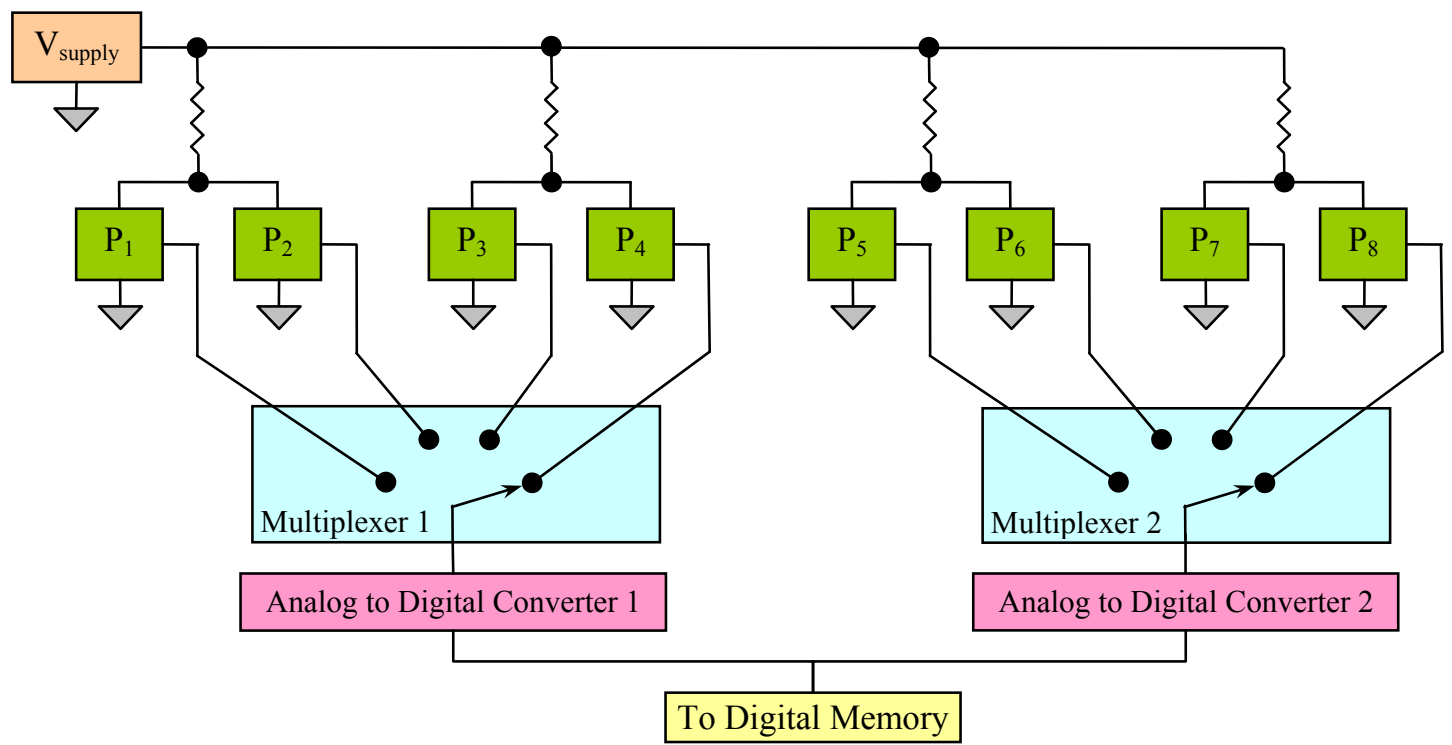

Fig. 27. Electronic pixel cross talk for a simplified, stylized eight-pixel $\left(\mathrm{P}_{1}-\mathrm{P}_{8}\right)$ FPA.

\section{Example 14: Nonuniformity}

Each pixel on the FPA has a different sensitivity to light. Also, the amplifiers in the analog-to-digital converters in Figure 27 may have slightly different gains and offsets. This is especially evident at short integration times, where nonuniformity becomes more severe. The process of correcting for these effects is called a NonUniformity Correction, or NUC. There are many schemes for performing a NUC ${ }^{(20-27)}$. Some are quite complex, and it is not always obvious how they affect camera calibration. 
One issue affecting which NUC procedure to use concerns whether the calibration and NUC of the system are viewed as two separate procedures or as one combined procedure. Figure 28 illustrates this. Since most thermal cameras have a field of view larger than the blackbody, off-the-shelf thermal camera software generally uses the two step method shown in Figure 28. Our system uses custom software which combines the NUC and calibration into one procedure, also shown in Figure 28. A series of images of a blackbody is acquired at various blackbody temperatures. The blackbody is in focus and fills the field of view for the portion of the FPA used. For each pixel, a set of cubic splines describing temperature as a function of camera response is generated. Thus, each set of cubic splines will function as a calibration curve for that pixel. These are stored in a data base. This process is repeated for each integration time used. After a cutting experiment has been performed, the software applies each calibration curve to the matching pixel for each frame in the movie. This converts the intensities in the thermal movie to imaged temperatures. Note that this is applied offline, well after the data has been acquired. Also, note that most off-the-shelf thermal camera software assumes the camera is being used in the linear portion of the FPAs response. Our custom software allows for as many calibration points as desired, enabling operation in the nonlinear portion of the FPA if needed.

When the in-focus field of view of the thermal image is larger
than the calibration source, the NUC and calibration must be
separated into two steps.

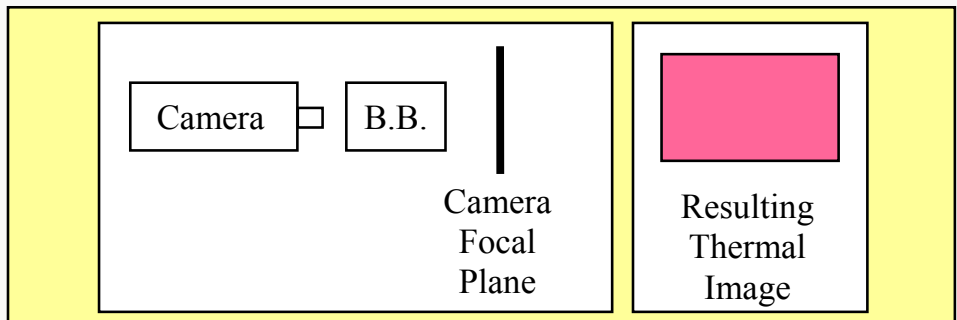

Step 1 - The blackbody is too small to both fill the field of view and be in focus at the same time. Bring the blackbody close to the camera to create a uniform image of unknown intensity which fills the field of view of the camera. Perform a NUC.

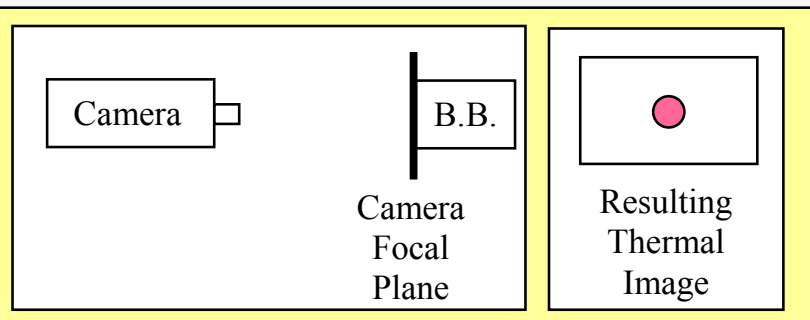

Step 2 - Move the blackbody to an in focus position. Perform a calibration of the NUCed images by using a small area of known intensity in the image to calibrate the entire image.
When the in-focus field of view of the thermal image is smaller than the calibration source, the NUC and calibration may be combined into one step.

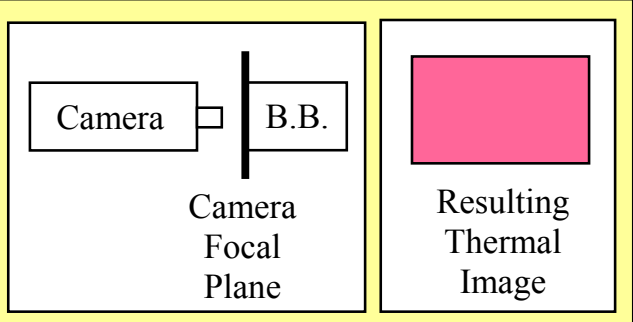

Step 1 - Since the intensity of this image is known and uniform, both the NUC and the calibration may be performed in one step.

Fig. 28. The field of view of the thermal camera, when compared to the size of the blackbody, determines whether the NUC and the calibration may be performed in one step or two.

When filling the entire field of view with a blackbody image, there are two issues to consider. One issue is to use an appropriately sized aperture, as discussed in Example 2. A second issue is to not "overwhelm" the FPA. As discussed in Example 13, the pixels share a power supply through circuits with resistances. If all the pixels are simultaneously exposed to very bright light, the voltages supplied to the amplifiers on the FPA may 
drop, effecting the calibration. However, we do not use the entire FPA in order to achieve higher frame rates. As shown in Figure 29, our optics allow light to fall on only a portion of the FPA, avoiding the problem.

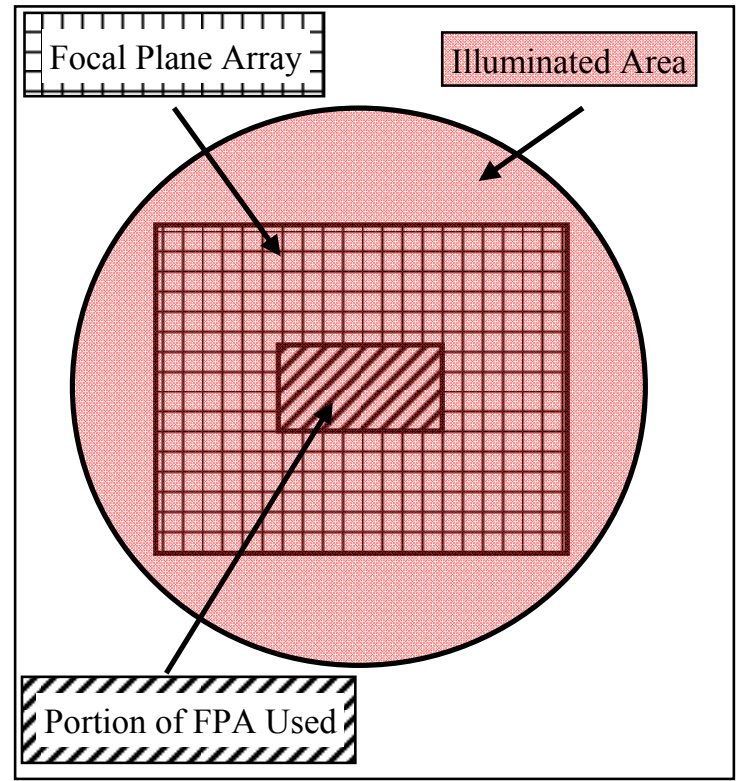

Light is filling the entire FPA, making the system vulnerable to overwhelming the FPA which effects calibration.

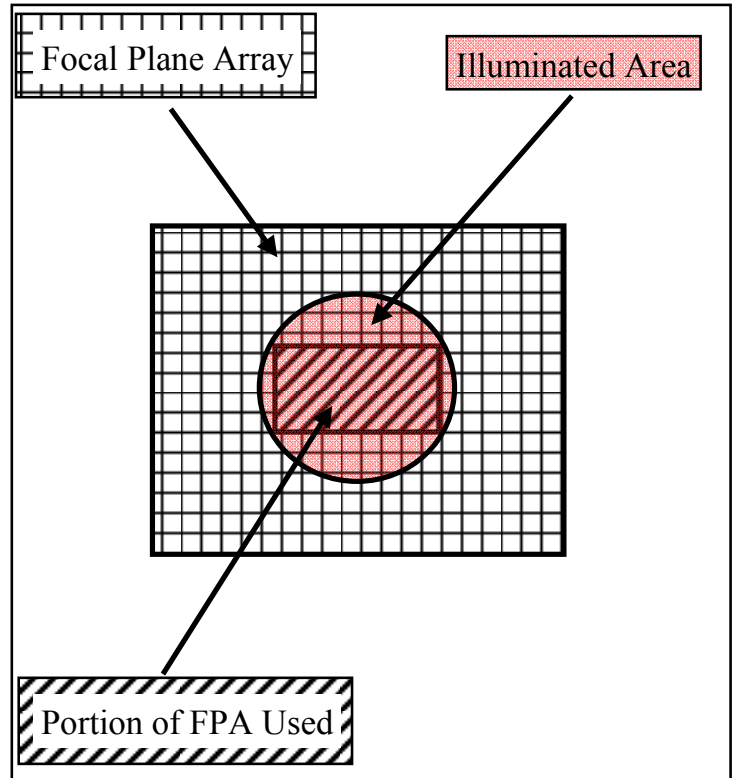

Our system illuminates only the portion of the FPA actually used. This minimizes any chance of the FPA being overwhelmed by bright images.

Fig. 29. Avoiding the problem of overwhelming the FPA.

As alluded to in Example 4, thermal camera software generally offers a "single point" and a "double point" option for the real-time NUC. When using the single point option, a single image is acquired and used to determine a constant to subtract from each pixel so that the image appears uniform. The slope of the calibration curve for each pixel is unchanged. This contrasts with the double point option, where a high- and a low-brightness reading are used to modify both the offset and slope for each pixel. Both readings should be in the linear portion of the FPA response. Acquisition of these readings is shown in Figure 30.

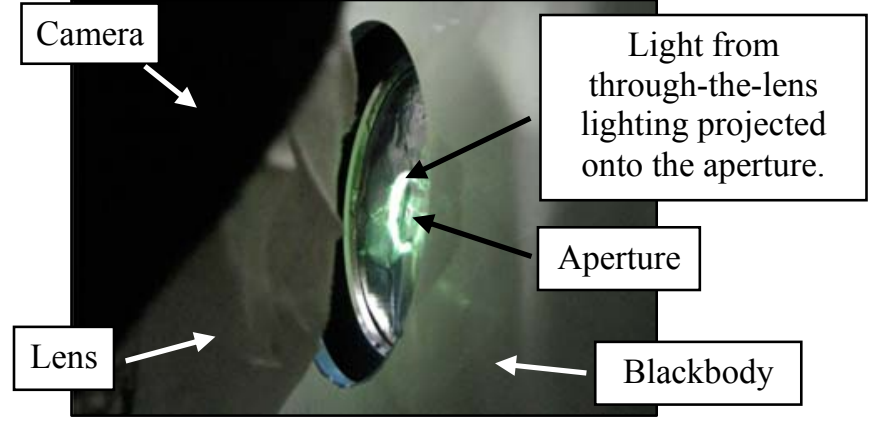

Through-the-lens lighting assists initial positioning of the thermal camera. A high temperature reading for the real-time NUC may now be acquired.

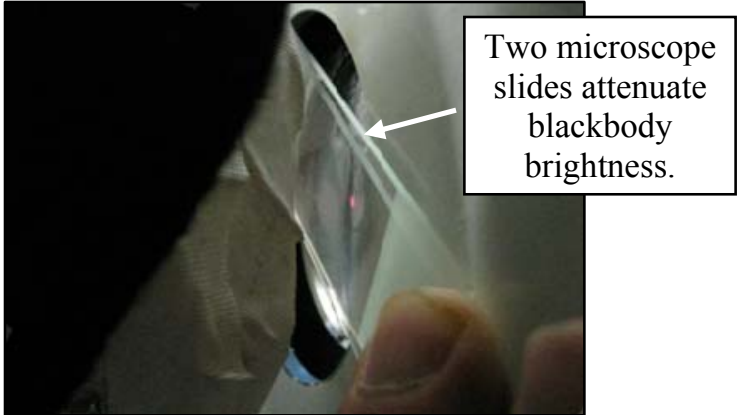

To acquire the low temperature reading, two microscope slides attenuate the intensity to prove a uniform, low intensity image within the linear range of the FPA.

Fig. 30. High- and low-brightness readings for the real-time NUC. 
A double point real-time NUC yields better results than a single point real-time NUC, but only if both points are in the linear portion of the camera response.

We have two NUC/calibration tools at our disposal. One tool is the real-time NUC and/or calibration provided by the camera software and the other tool is our custom offline software. Figure 31 shows four schemes for applying these tools. The scheme shown in Figure 31a uses only the real-time NUC and calibration provided by the camera software. We desired the ability to operate outside the linear range of the camera, so we rejected this scheme. In Figure 31b, only our custom software is used. The problem with this scheme is that the real-time display is almost unusable, due to the highly nonuniform images acquired when using very short integration times. In Figure 31c, both tools are used. Unfortunately, this scheme is unnecessarily confusing to the user as there are three different appearances to the same data set - the real-time data looks different from the stored data, which looks different from the data after it has been processed by the offline NUC/calibration tool. Figure 31d shows the scheme we use for MADMACS. Here, the real-time display looks the same as the stored data, which is less confusing to the operator. However, since the real-time NUC and/or calibration affect the input to the offline NUC/calibration tool, care must be taken that the same real-time NUC and/or calibration is used for every test.

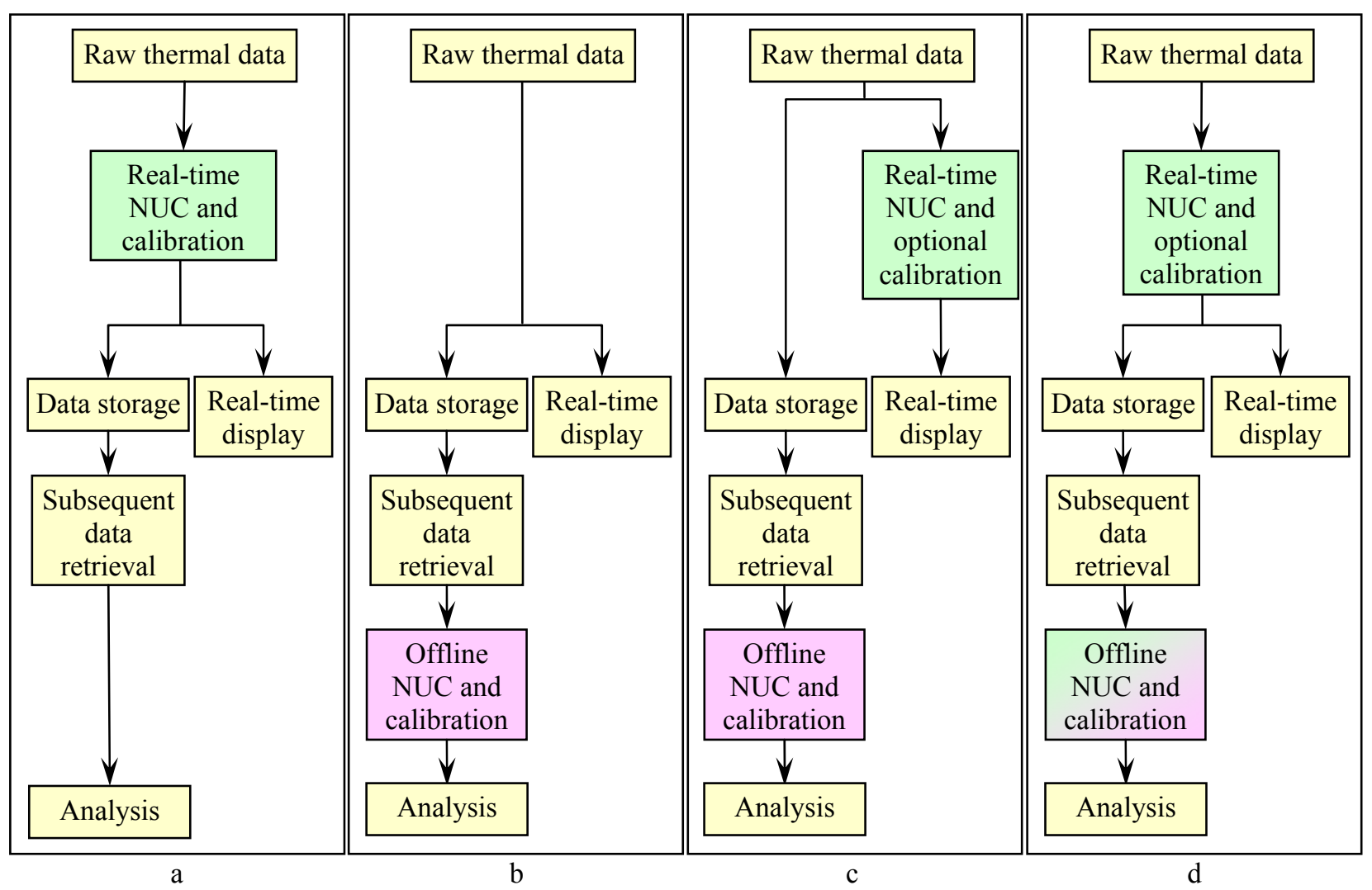

Fig. 31. Various schemes for applying a NUC and calibration.

\section{Example 15: Timing uncertainties}

There are several sources of timing uncertainties. One, sometimes referred to as timing jitter, is when there is large variation in either the time intervals between frames or the integration times of the frames. Another is the phenomenon of dropped frames, where the hardware is unable to process the video stream rapidly, 
resulting in frames becoming lost. Figure 32 shows two common hardware schemes. Figure 32a shows a scheme typical in high-speed visible light cameras. The digital memory is dedicated to data acquisition, so it does a good job of storing frames at a high rate of speed. Also, having the memory connected directly to the sensor improves speed as the long cable connecting the camera to the computer is sometimes a bandwidth bottleneck. The stream is saved to the computer hard drive after the test is over. This contrasts with the scheme shown in Figure 32b, which is common in thermal cameras as well as medium-speed and low-speed visible light cameras. Here, a general purpose computer is relied upon to capture the data stream in real time. If the camera outputs frames faster than either the computer memory or hard drive can process them, frames will be lost. Each lost (dropped) frame corresponds to a timing jitter of $100 \%$. Some cameras support time stamping each frame, which helps to detect dropped frames. Unfortunately, most cameras do not. Even with a fast computer, the scheme in Figure 32a typically outperforms the scheme in Figure 32b.
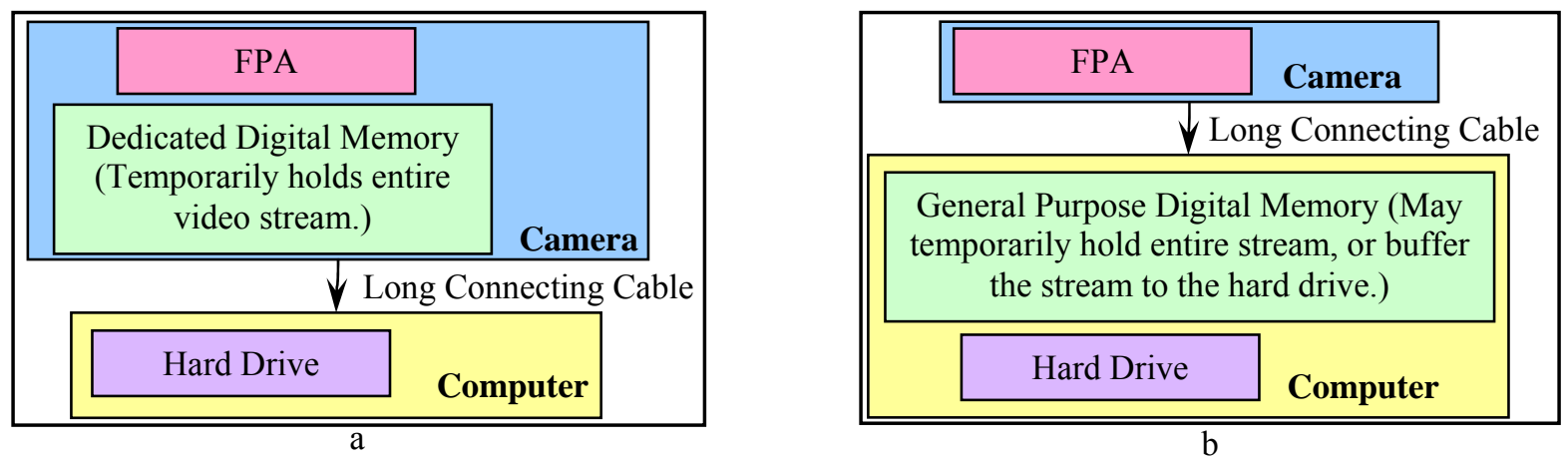

Fig. 32. Two common hardware schemes for acquiring video streams.

\section{Example 16: Persistence.}

As discussed in Example 13, if a pixel is exposed to very bright light and saturates, it takes time to recover. In addition, even when not saturated, all electronic devices have a limit on how fast they can change their output voltage or current. This is especially easy to detect in a high-speed visible light camera set to either a high gain or a low gamma (high gain at low light levels). Figure 33 shows four visible light images of a backlit, $200 \mu \mathrm{m}$ diameter aperture mounted near the edge of a rotating wheel. Two surface speeds and two integration times are shown. Figure 33d shows both motion blur (discussed in Example 19) and persistence. It is likely that thermal cameras are affected in a similar way.

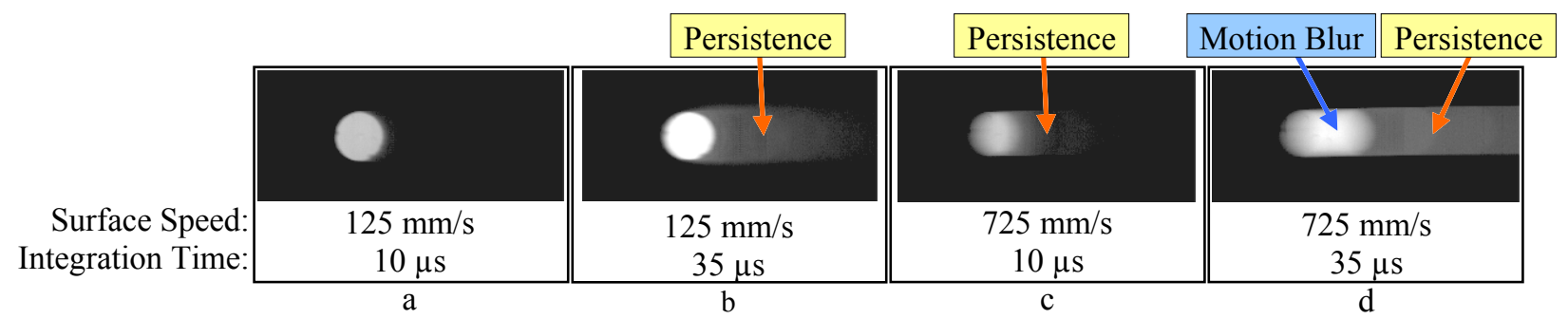

Fig. 33. Visible light images of a backlit $200 \mu \mathrm{m}$ diameter aperture mounted near the edge of a rotating wheel. 


\subsection{Other issues}

There are uncertainty sources not discussed above. They may directly affect amplitude, temporal, and spatial uncertainties in temperature measurements. Three examples are given below.

\section{Example 17: Reflections}

As shown in Figure 34, if light emitted by a hot surface is reflected by a second surface, the second surface may appear hotter than it actually is. Thus, it is important to know the relative positions of hot surfaces to properly interpret thermal images.

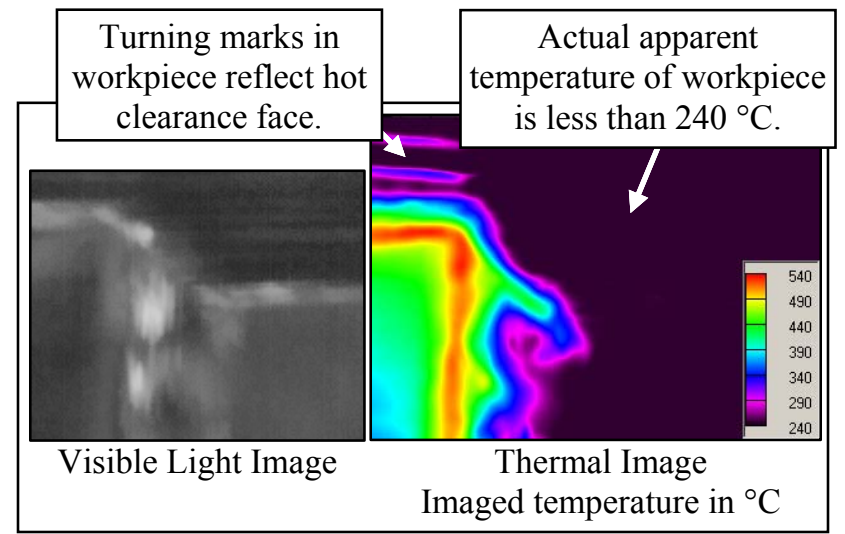

If the hot tool protrudes too far in front of the workpiece, the tool can be reflected in the workpiece.

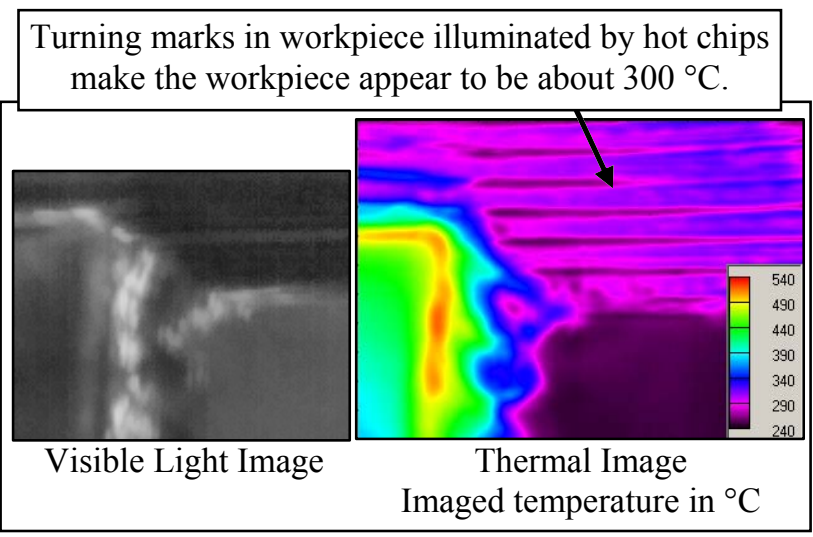

Hot, flying chips may sometimes also be reflected.

Fig. 34. Two examples, acquired only milliseconds apart, of reflections effecting imaged temperature. 


\section{Example 18: Are surface temperatures an accurate measure of internal temperatures?}

Thermal imaging yields the temperature on the surface of an object. Researchers typically assume that the surface temperature is indicative of the temperature inside the object. To verify this assumption for a cutting tool, grooves were cut into a tool as shown in Figure 35. Thermocouples were then cemented into the grooves with high temperature cement. The cement was selected to have a thermal conductivity and thermal expansion similar to that of the cutting tool. The temperature sensitive portions of the thermocouples were positioned so that they are approximately where the center of the chips will be during machining, i.e., slightly more than half the chip width from the imaged side of the tool. It should be noted that the grooves significantly weaken the tool. Thus, aggressive cutting conditions can not be used with this tool.

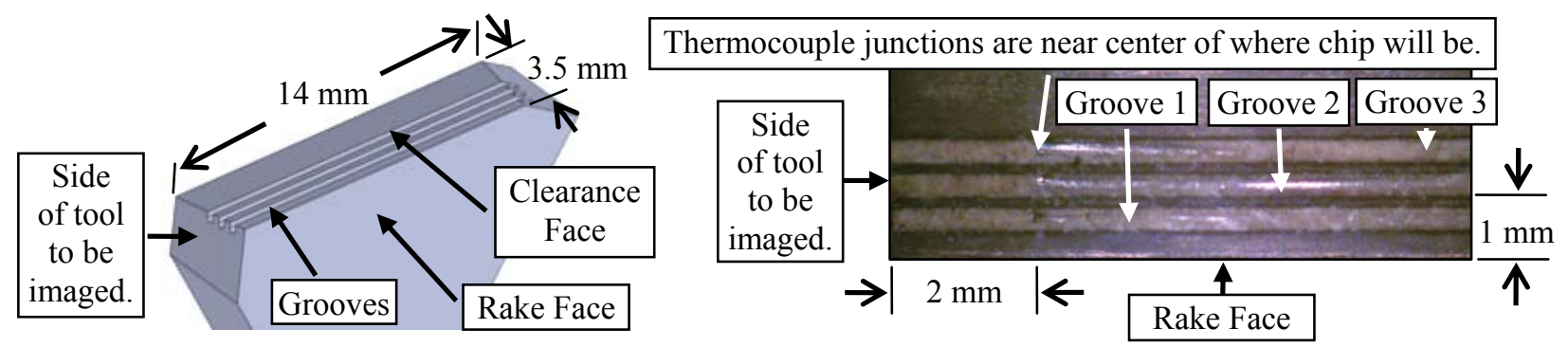

Fig. 35. Isometric view (drawing on left) and top view (photo on right) of grooves in the cutting tool.

Figure 36 shows the instrumented tool temporarily wrapped in aluminum foil and heated by thermal tweezers. A thermal image is acquired after 10 minutes. This serves several purposes. First, water in the cement is driven off. Second, an oxide layer is formed on the tool and the cement, preventing changes in emissivity during the cutting experiments. Finally, comparing the thermal image to the true temperature of the tool allows the emissivity of the cement in the grooves to be measured.

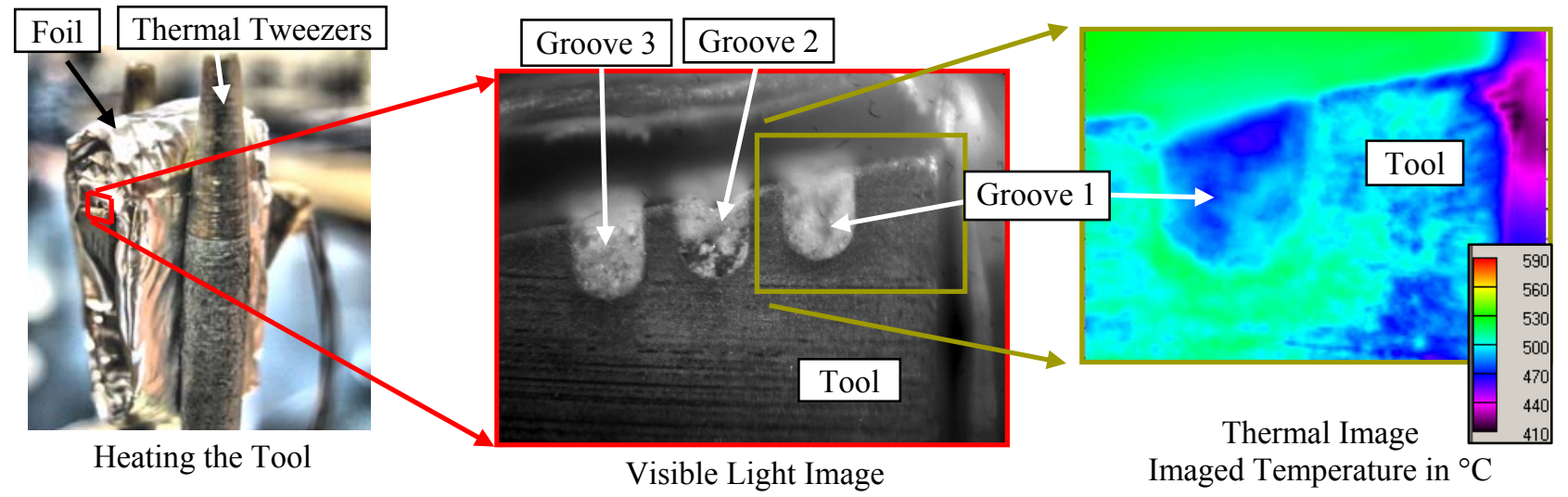

Fig. 36. Heating instrumented cutting tool in thermal tweezers.

The instrumented tool was mounted and a cutting experiment performed with a $3.2 \mathrm{~mm}$ wide steel workpiece. Only Groove 1 was imaged in this experiment. Groove 2 and Groove 3 are used in a different experiment. In contrast to the chip, the tool is stationary in the images, and the surface is relatively uniform. Also, we are only concerned with an average temperature in the relatively large area of the cement filled groove. 
Therefore, there is no need to correct for the PSF of the camera or for motion blur in the images. Video files of this experiment are available on the NIST website ${ }^{(9)}$.

Figure 37 shows the true temperature of the cement reported by the thermal camera, as well as the thermocouple temperature. There is good agreement during the central portion of the test and less agreement during other portions. There are several possible explanations for this behavior which are currently being explored. This data indicates that a thermal imaging measurement of the side of the cutting tool is representative of the temperature within the tool, at least some of the time.

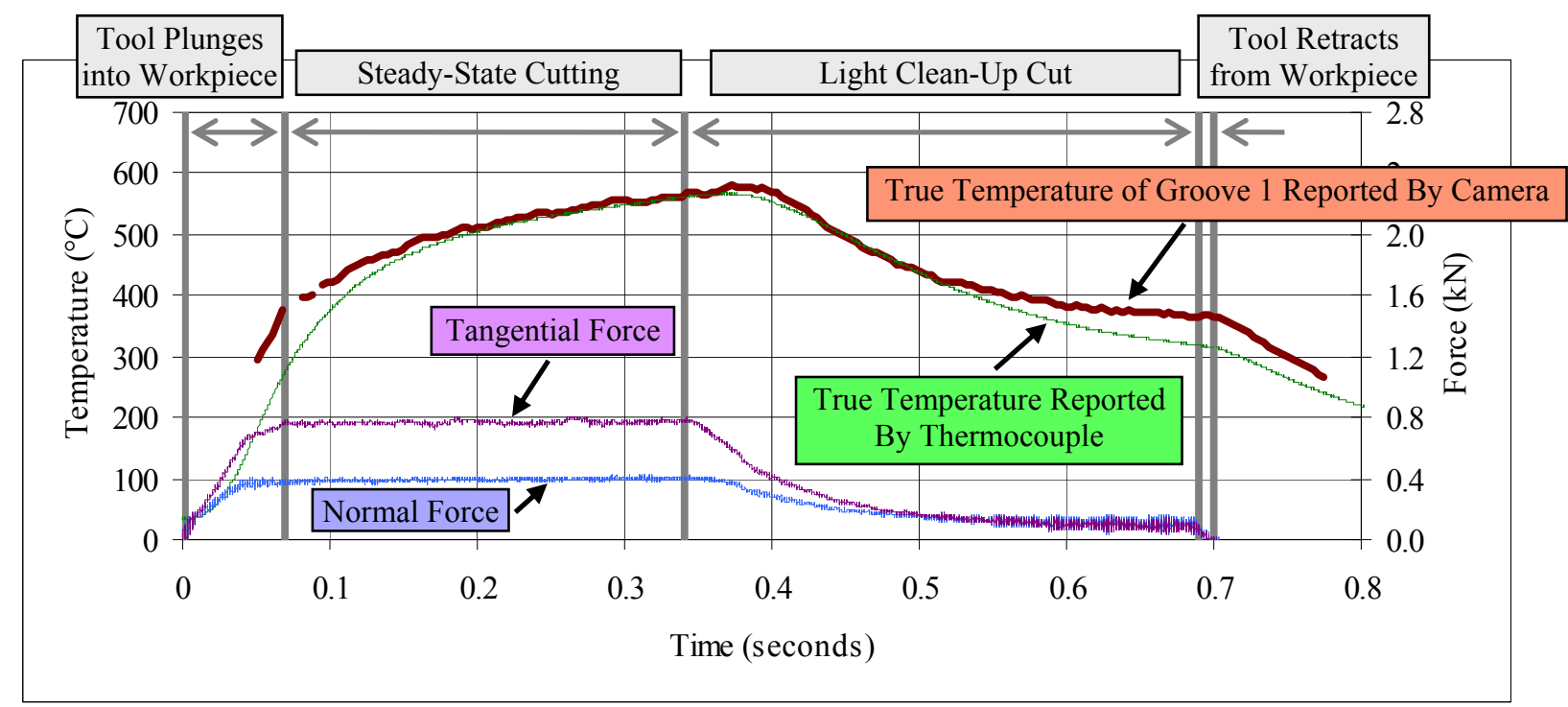

Fig. 37. Comparing thermocouple and camera temperatures. Cutting forces indicate when cutting at the full depth of cut occurred (between about $0.07 \mathrm{~s}$ and $0.34 \mathrm{~s}$ ). Cutting between $0.34 \mathrm{~s}$ and $0.69 \mathrm{~s}$ is a light cut which ensures the disk is circular for the next test.

\section{Example 19: Motion blur, size-of-source effects, and insufficient temperature range.}

In real-world situations, multiple uncertainty sources often act together to affect a measured value. When imaging the metal cutting process, some features of interest may range from $0.1 \mu \mathrm{m}$ to $50 \mu \mathrm{m}$ in size, such as the thickness of the shear zone. A variety of factors makes measuring the temperatures of such small features problematic. One factor is that spatial resolution is limited by the wavelength of light used to image the feature $^{(15)}$. Visible light cameras are sensitive to wavelengths of less than $1 \mu \mathrm{m}$, while thermal cameras are sensitive to wavelengths up to $24 \mu \mathrm{m}$. This is the primary reason visible light cameras are generally able to image smaller features than thermal cameras. Also, if a feature is so small that it does not completely fill a pixel, the measured amplitude will be incorrect ${ }^{(15)}$. In addition, imperfections in the optics are more noticeable when imaging small features. One method for describing how the aforementioned effects influence the camera image is the point spread function (PSF), which describes the response of an imaging system to a point source. It should be noted that diffraction effects ${ }^{(15 ; 28)}$ are also possible, but are ignored in this paper. 
As discussed in Example 10, the PSF for our camera optics is the dominant size-of-source effect. The PSF for the camera system was measured using the knife edge technique, and is shown in Table 4.

\begin{tabular}{|c|c|}
\hline Lateral Displacement (Thermal Camera Pixels) & PSF (Normalized So That Area $=1)$ \\
\hline 0 & 0.254 \\
\hline$-1,+1$ & 0.197 \\
\hline$-2,+2$ & 0.124 \\
\hline$-3,+3$ & 0.051 \\
\hline$-4,+4$ & 0.001 \\
\hline
\end{tabular}

Table 4. PSF as a function of lateral displacement for MADMACS.

Figure 38 shows a one-dimensional model for the effects of the PSF and motion blur on the imaged intensity data. Appendix 4 provides a Mathcad $\AA$ implementation of the model. The undistorted, true intensity curve is modeled as a bar function representing a shear zone of width $W_{\mathrm{SZ}}$ and height $I_{\mathrm{SZ}}$, surrounded by a constant value $I_{\mathrm{CF}}$ representing the intensity of the chip faces (term A of the convolution). This curve is convoluted with the normalized point spread function for the camera (term B) and a normalized bar of width $W_{\mathrm{MB}}$ representing the distance traveled by the shear zone during the integration time of the camera (term $\mathrm{C}$ ). The area under both the point spread function (term B) and the motion blur curve (term C) is 1 , so the area under the shear zone and chip face intensity curve (term A) is not altered by the convolution. However, the convolution will reduce the height and increase the width of the modeled shear zone intensity data.

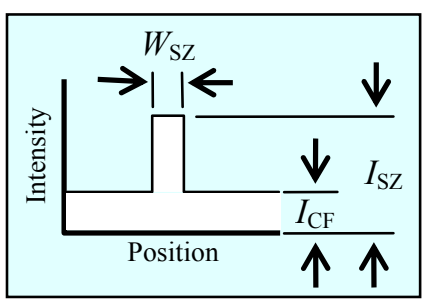

Shear Zone and Chip Face (Term 'A')

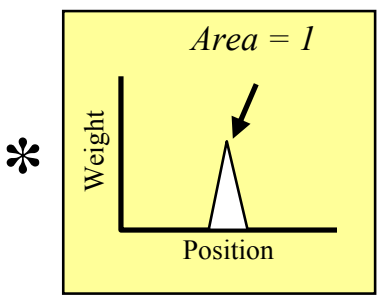

Point Spread Function (Term 'B')

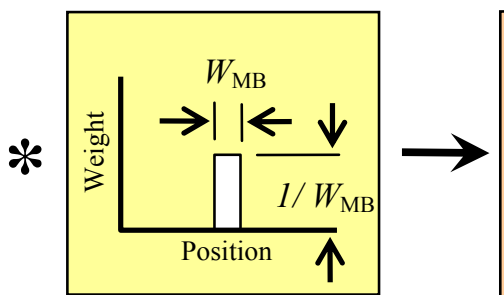

Motion Blur

(Term 'C')

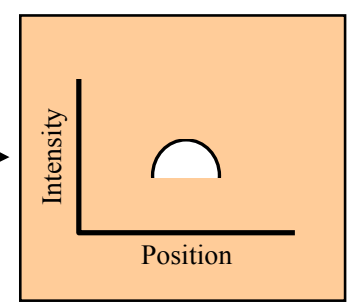

Imaged Intensity Data

(Result of Convolution)

Fig. 38. Schematic of modeling imaged intensity data as a convolution of the emitted intensity (A), the camera PSF (B), and motion blur (C).

In theory, if one knows the PSF and $W_{\mathrm{MB}}$, they may be deconvolved from the measured intensity data to compute the shear zone and chip face intensity curve. However, there are two difficulties with this approach. First, deconvolution is very sensitive to noise in the data. Computed results vary wildly in response to even small changes in the input data. Second, the imaged intensity data is often an incomplete data set. The reason that it may be an incomplete data set is that the chip face is often much cooler than the shear zone. In order to have enough sensitivity to measure the chip face temperature, the integration time must be increased. However, increasing the integration time also increases the motion blur. If the integration time is made too long, the motion blur can become so severe that the thermal image lacks sufficient detail. Thus, one often does not have data for the cooler portions of the measured intensity data.

An alternative approach is to select values for $W_{\mathrm{SZ}}, I_{\mathrm{SZ}}, I_{\mathrm{CF}}, W_{\mathrm{MB}}$, and the PSF. The convolution is computed and compared to the imaged intensity data. If there is good agreement, the selected values are plausible. One advantage to this approach is that sensitivity analysis may be performed. For example, if changing $I_{\mathrm{SZ}}$ by a large amount has little effect on how well the computed convolution matches the imaged data, uncertainty in $I_{\mathrm{SZ}}$ is large. 
Figure 39 shows a data set of segmented titanium chip formation we will analyze using this approach. Note that the thermal camera was insensitive to temperatures lower than $240{ }^{\circ} \mathrm{C}$. The face of the chip is $240{ }^{\circ} \mathrm{C}$ or cooler while the shear zone is over $350{ }^{\circ} \mathrm{C}$. To estimate $W_{\mathrm{MB}}$ (MotionBlurWidth in Appendix 4) successive visible light images were examined. To create a one-dimensional imaged intensity data set, temperatures were sampled along a line through the shear zone in the thermal image. In addition to the data derived from Figure 39, the PSF for the camera was used ( $p s p$ in Appendix 4). The worksheet in Appendix 4 allows a user to input values for $W_{\mathrm{SZ}}$ (ShearZoneWidth in Appendix 4) and the temperature which corresponds to $I_{\mathrm{CF}}$ (BaseLineTemperature in Appendix 4). It then solves the convolution, setting the value of $I_{\mathrm{SZ}}$ (ShearZoneIntensity in Appendix 4) so that the area under the computed convolution curve is equal to the area under the imaged intensity curve. The Root Mean Square (RMS) value of the residuals (differences between imaged values and results of the convolution) is also generated.

To illustrate the importance of knowing $W_{M B}$, we will momentarily ignore that we measured it using data from Figure 39. Figure 40 shows results where we assume $I_{\mathrm{CF}} \cong 240{ }^{\circ} \mathrm{C}$ and a range of $W_{\mathrm{MB}}$ and $W_{\mathrm{Sz}}$ are tried. The height of the peak imaged shear zone temperature is shown as a horizontal line. Any valid value for $T_{\mathrm{SZ}}$ (the temperature corresponding to $I_{\mathrm{SZ}}$ ) must be at or above this line. Notice that at each $W_{\mathrm{SZ}}$, the values for $T_{\mathrm{SZ}}$ are nearly the same and seem like a single point in the plot, while the RMS values of the residuals are clearly different. Assume that when the RMS of the residuals is a minimum, the corresponding value of $T_{\mathrm{SZ}}$ is the most likely to be the correct value. Thus, when $W_{\mathrm{MB}}=41 \mu \mathrm{m}, T_{\mathrm{SZ}} \approx 660{ }^{\circ} \mathrm{C}$ and when $W_{\mathrm{MB}}=16 \mu \mathrm{m}$, $T_{\mathrm{SZ}} \approx 370{ }^{\circ} \mathrm{C}$. Not knowing $W_{\mathrm{MB}}$ results in large uncertainty in our estimate of $T_{\mathrm{SZ}}$.

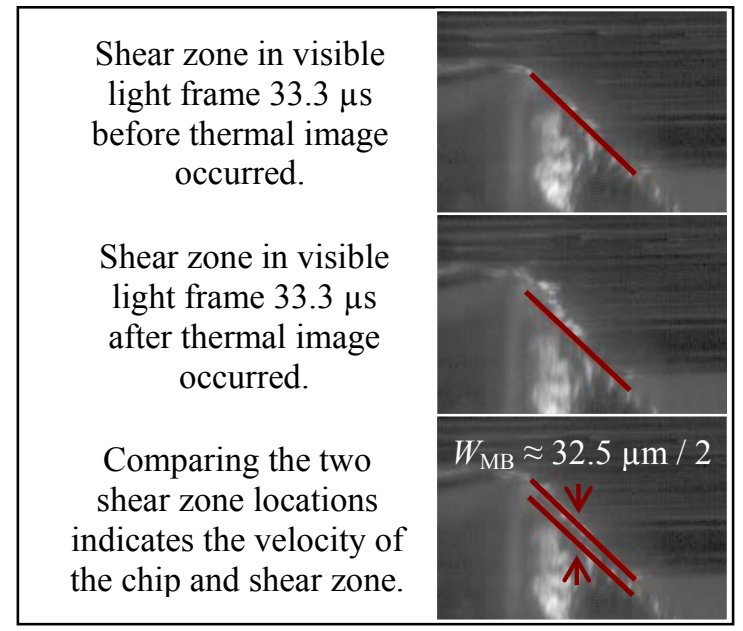

Visible Light Images

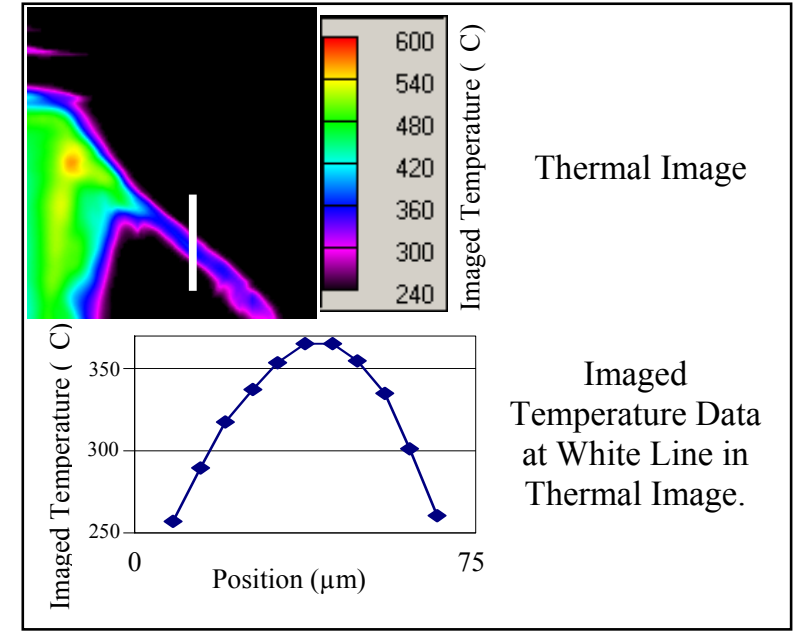

Thermal Data

Fig. 39. Visible and thermal spectrum images used to estimate $W_{\mathrm{MB}}$ and imaged temperature data.

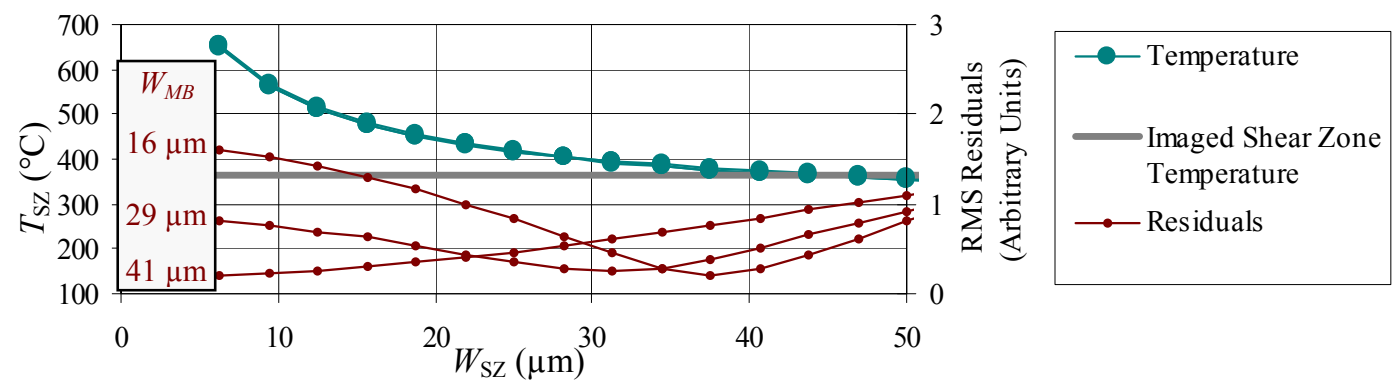

Fig. 40. Results where $I_{\mathrm{CF}} \cong 240{ }^{\circ} \mathrm{C}$ and $W_{\mathrm{MB}}$ is assumed to be unknown. 
It is interesting to note that the primary factor determining whether the computed convolution is a good fit to the measured image intensity (small RMS of the differences) is how well the widths of the two curves match. This is illustrated by the example shown in Figure 41.

In Figure 42, we take advantage of our measured value for $W_{\mathrm{MB}}$. Three values for $W_{\mathrm{MB}}$ are tried, $10 \mu \mathrm{m}$, $16.25 \mu \mathrm{m}$, and $22.5 \mu \mathrm{m}$. These values correspond to the value for $W_{\mathrm{MB}}$ measured in Figure 40, as well as +1 thermal image pixel (+1.25 visible image pixel) and -1 thermal image pixel ( -1.25 visible image pixel). It is assumed that we have measured $W_{\mathrm{MB}}$ to this level of accuracy. We assume $I_{\mathrm{CF}}$ corresponds to either $240{ }^{\circ} \mathrm{C}$ or $130{ }^{\circ} \mathrm{C}$. $I_{\mathrm{CF}} \cong 20^{\circ} \mathrm{C}$ was also tried and yielded results similar to $130{ }^{\circ} \mathrm{C}$, so results for $20^{\circ} \mathrm{C}$ are not shown. The range of likely $T_{\mathrm{SZ}}$ is shown in the two gold colored ovals in Figure 42 . We can safely say that $T_{\mathrm{SZ}}$ (the apparent temperature of the shear zone) is between $366{ }^{\circ} \mathrm{C}$ and $384{ }^{\circ} \mathrm{C}$. Emissivity may be determined after the cutting test ${ }^{(29)}$ and used to convert this range into a range of plausible true temperatures for the shear zone.

Note that if $I_{\mathrm{CF}} \equiv 240{ }^{\circ} \mathrm{C}$, the narrower $(34 \mu \mathrm{m}$ to $41 \mu \mathrm{m})$, hotter $\left(368{ }^{\circ} \mathrm{C}\right.$ to $\left.384{ }^{\circ} \mathrm{C}\right)$ shear zone values are more likely to be correct. If $I_{\mathrm{CF}} \cong 130^{\circ} \mathrm{C}$, the wider $(50 \mu \mathrm{m})$, cooler $\left(366^{\circ} \mathrm{C}\right.$ to $\left.368^{\circ} \mathrm{C}\right)$ shear zone values are more likely. Knowing the chip face temperature is important information - even if the shear zone temperature is the ultimate goal. A repeat test, with the same cutting conditions but a long enough integration time to capture the chip face temperature, may be useful here. This data indicates that motion blur and PSF effects may be more accurately compensated for if other information, such as chip face temperature, is measured.

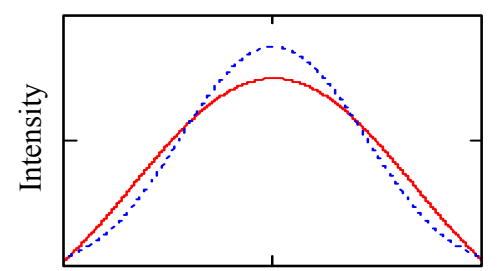

Position (63 $\mu \mathrm{m}$ full scale)

$W_{\mathrm{SZ}}=10 \mu \mathrm{m}$

RMS of differences is 0.7

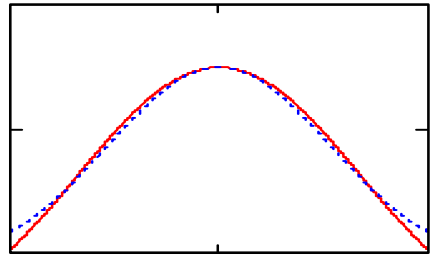

Position $(63 \mu \mathrm{m}$ full scale $)$

$W_{\mathrm{SZ}}=33 \mu \mathrm{m}$

RMS of differences is 0.3

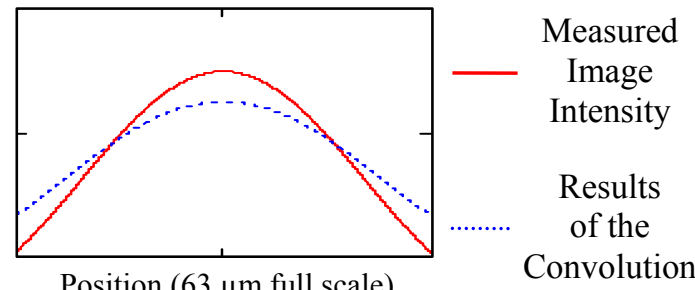

Position $(63 \mu \mathrm{m}$ full scale $)$

$W_{\mathrm{SZ}}=50 \mu \mathrm{m}$

RMS of differences is 0.9

Fig. 41. Illustrative example showing that the lowest RMS of the differences is obtained when the width of the measured image intensity curve matches the width of the resulting convolution. $I_{\mathrm{CF}} \cong 240^{\circ} \mathrm{C}$ and $W_{\mathrm{MB}}=29 \mu \mathrm{m}$.
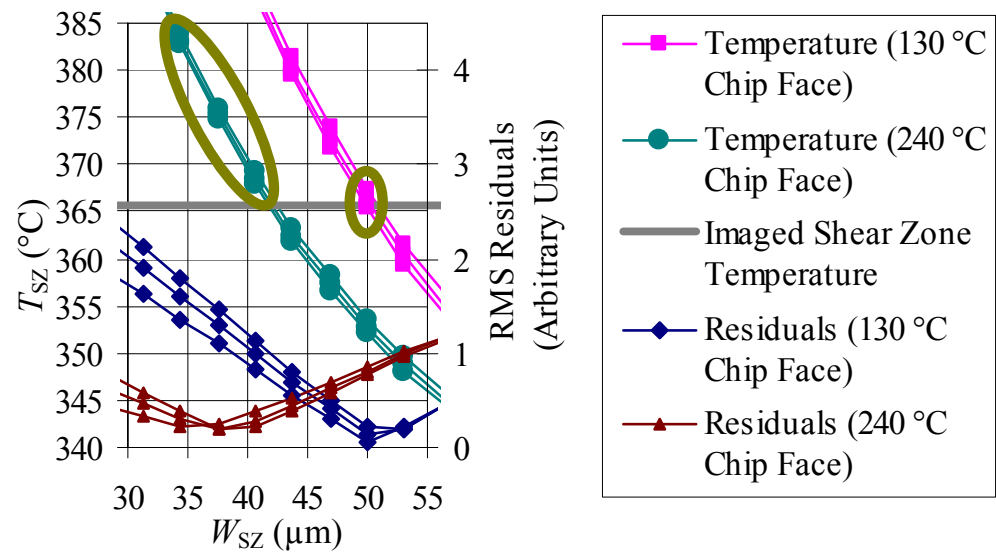

Fig. 42. Results where $I_{\mathrm{CF}}$ is assumed to correspond to either $130{ }^{\circ} \mathrm{C}$ or $240{ }^{\circ} \mathrm{C}$, and $W_{\mathrm{MB}}$ is the measured value, the measured value +1 thermal image pixel, and the measured value -1 thermal image pixel. 
This example showed a titanium chip and had little uncertainty. This is in part due to the surface speed of the workpiece (and the chip) being relatively low. However, many materials are cut using much higher surface speeds. In these cases, the uncertainty is significantly worse due to increased motion blur. Also, this example showed a segmented chip. The location of the shear zone generally coincides with the location of the gap between segments. This has the effect of widening the shear zone. NIST is currently exploring unsegmented, continuous chips. It is suspected that continuous chips may have thinner shear zones. If this is the case, the uncertainty due to the PSF of the camera will be larger than in this example.

\section{FUTURE DIRECTIONS}

Thermal imaging of machining is performed "dry", without cutting fluid. Measured temperatures may then be compared to modeling results for dry cutting to verify the model. This avoids the problem of the cutting fluid interfering with imaging the cutting process. Minimal Quantity Lubrication (MQL) technology, or even blowing cold inert gas on the workpiece during cutting, may help get thermal readings at conditions closer to lubricated cutting.

By using a slotted disk for the workpiece, NIST has performed preliminary tests with interrupted cutting. Such tests are more like milling than when using a solid disk. High-speed visible light video files are available at the NIST website ${ }^{(9)}$.

DIC, or Digital Image Correlation may be performed on visible light images to create displacement vectors describing how the material moves ${ }^{(4)}$. Briefly, this is accomplished by first breaking a frame into small regions. For each region, that same region in the subsequent frame is found using a correlation criterion. How far each region moved from one frame to the next may be described by displacement vectors. An example is shown in Figure 43. Once displacement vectors have been determined, they may be converted into velocity, acceleration, strain, or strain rate. Examples of DIC are available on the NIST website ${ }^{(9)}$.

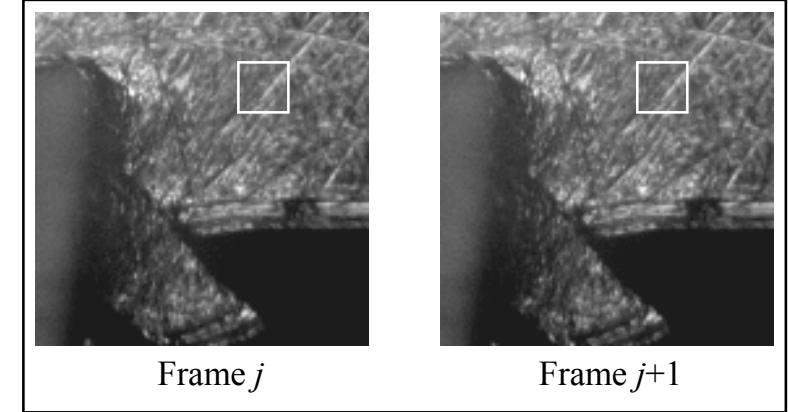

Comparing frame $j$ to frame $j+1$, the white square has moved to the left by approximately 2 pixels.

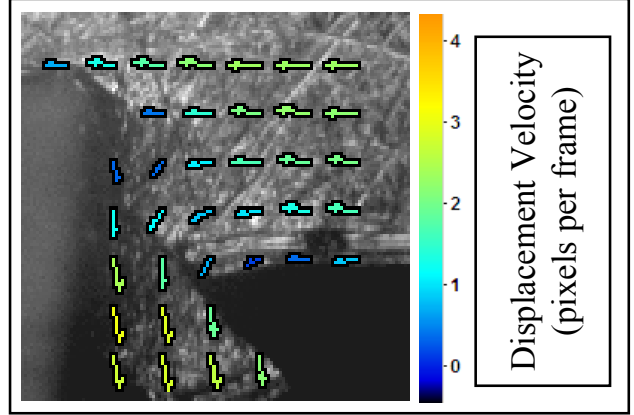

Repeatedly applying this process generates a set of displacement vectors. Note that the vectors are rendered with exaggerated lengths to make them easier to see in the above figure.

Fig. 43. Displacement vector map shows how features moved from one frame to the next. 
While visible light images from the dual-spectrum system are of lower quality than the images obtained with a visible light camera which were used in the earlier NIST DIC research ${ }^{(4)}$, it is hoped that visible light images from the dual-spectrum system will be of sufficient quality to implement the procedure shown in Figure 44. The intent is to use image processing techniques to merge visible light images, thermal images, and other information to improve the quality and information content of both the visible and thermal spectrum images and minimize the impact of many of the uncertainties discussed in this report. Combining images of different spectrums is sometimes referred to as multispectral imaging or image fusion ${ }^{(30 ; 31)}$ and may significantly lower temperature uncertainty due to uncertainty in emissivity values. Combining image data with other forms of data, such as cutting forces, is sometimes called data fusion. Both are active areas of research.

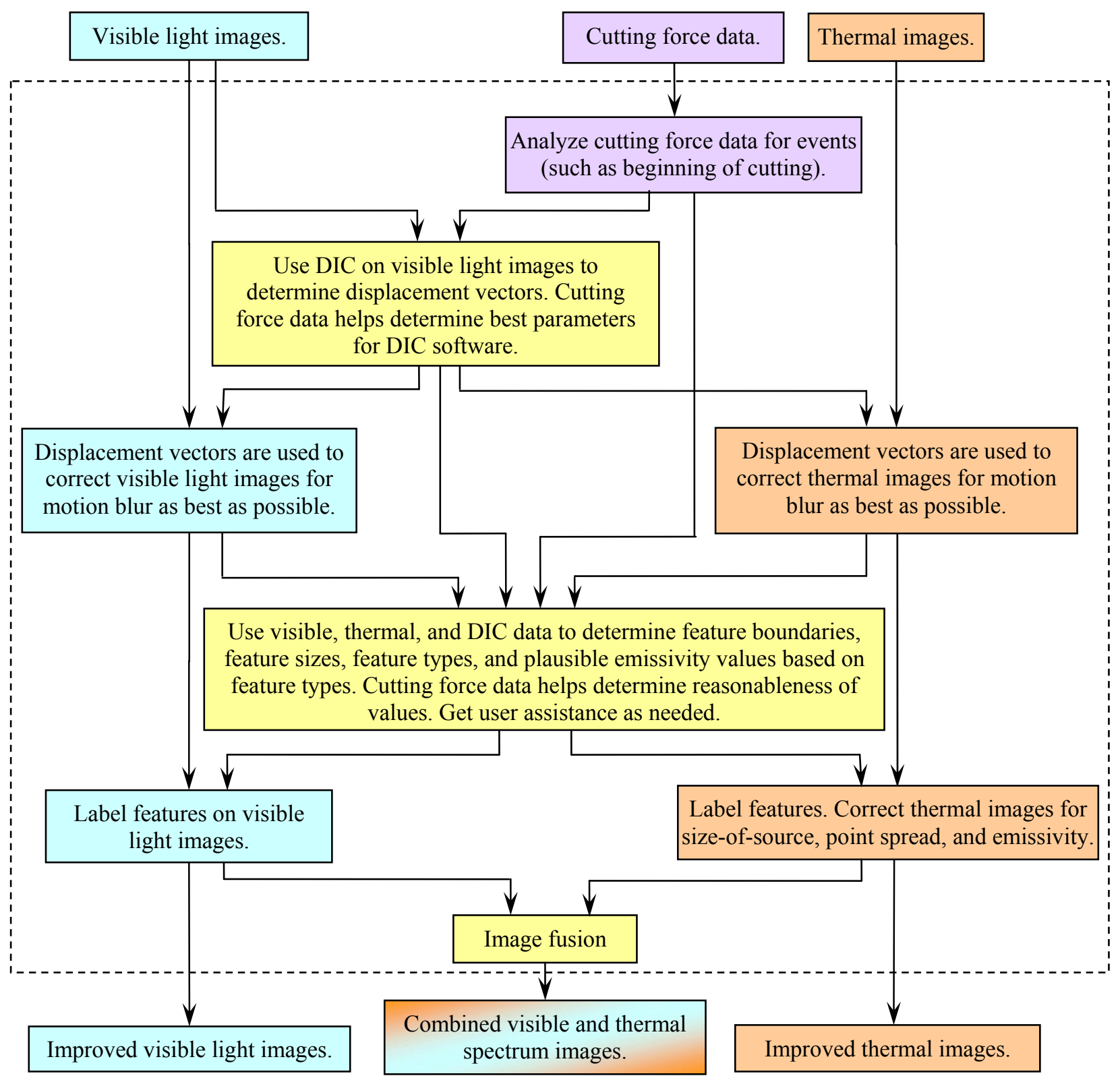

Fig. 44. A procedure for combining thermal spectrum, visible spectrum, displacement vector, and cutting force data to improve the images. 


\section{CONCLUSIONS}

Dual-spectrum imaging is a powerful technique for enabling a better understanding of the metal cutting process. However, many uncertainty sources need to be understood to correctly acquire and interpret the data. In this report, uncertainty sources were divided into five categories: calibration of camera sensitivity and offset, conversion of apparent to true temperature, camera optics, electronic effects, and other issues. Generalities for each category were presented, along with specific illustrative examples. To determine whether an uncertainty source may affect any given measurement, one must keep in mind that temperature measurements describe a temperature at a location over some time interval. Thus, any measurement has several related uncertainties: amplitude, temporal (time) and spatial (location in space). Each uncertainty may interact to affect the final uncertainty of the measurement. Digital image correlation and image processing techniques hold the potential to automate the analysis of such data.

\section{ACKNOWLEDGEMENTS}

The author wishes to acknowledge Timothy Burns, April Cooke, Jarred Heigel, Robert Ivester, Richard Rhorer, Johannes Soons, Benjamin Tsai, and Howard Yoon of the National Institute of Standards and Technology; as well as Ivan Arriola of Mondragon University, Spain.

\section{REFERENCES}

[1] Ivester, R. W. and Heigel, J. C., "Smart Machining Systems: Robust Optimization and Adaptive Control Optimization for Turning Operations," Transactions of the North American Research Institute (NAMRI)/SME, Vol. 35, 2007, pp. 505-511.

[2] Whitenton, E. P., Ivester, R. W., and Yoon, H., "Simultaneous Visible and Thermal Imaging of Metals During Machining," SPIE Proceedings, Vol. 5782, 2005, pp. 71-82.

[3] Heigel, J. C., Ivester, R. W., and Whitenton, E. P., "Cutting Temperature Measurements of Segmented Chips using Dual-Spectrum High-Speed Microvideography," Transactions of the North American Research Institute (NAMRI)/SME, Vol. 36, 2008, pp. 73-80.

[4] Whitenton, E. P., Heigel, J. C., and Ivester, R. W., "Measurement and Characterization of Dynamics in Machining Chip Segmentation," 11th CIRP International Workshop on Modeling of Machining Operations, 2008, pp. 237-246.

[5] Ivester, R. W., Whitenton, E. P., Heigel, J. C., Marusich, T., and Arthur, C., "Measuring Chip Segmentation by High-Speed Microvideography and Comparison to Finite-Element Modeling Simulations," 10th CIRP International Workshop on Modeling of Machining Operations, 2007, pp. 3744.

[6] Zhang, Z. M., Tsai, B. K., and Machin, G., Radiometric Temperature Measurements: II. Applications, Elsevier Academic Press, New York 2010. 
[7] Saunders, P., Radiation Thermography, Fundamentals and Applications in the Petrochemical Industry, SPIE Press, 2007.

[8] Muller, B. and Renz, U., "Thermographic Measurements of High-Speed Metal Cutting," SPIE Proceedings, Vol. 4710, 2002, pp. 126-134.

[9] Whitenton, E. P.. High-Speed and Dual-Spectrum Videos of Machining Processes. Current URL http://nist.gov/mel/mmd/mpm/hsds-machining-videos.cfm Future URL (due to re-organization) http://nist.gov/el/isd/sbm/hsds-machining-videos.cfm . 2010.

[10] Heigel, J. C. and Whitenton, E. P., "High-Speed Microvideography Observations Of The Periodic Catastrophic Shear Event In Cutting Aisi 1045 Steel," Transactions of NAMRI/SME, Vol. 37, 2009, pp. $33-40$.

[11] Gnanamanickam, E., Huang, C., Murthy, T. G., Sullivan, J. P., and Chandrasekar, S., "In Situ Measurement of Deformation and Temperature in Machining," 12th CIRP Conference on Modelling of Machining Operations, 2009, pp. 384-388.

[12] Taylor, B. and Kuyatt, C., "NIST Technical Note 1297, Guidelines for Evaluating and Expressing the Uncertainty of NIST Measurement Results," 1994.

[13] Distl, R. and Egeler, T., "Polarization Measurement of Light Radiation," Report by Instrument Systems, Germany, 1988.

[14] Vollmer, M., Henke, S., Karstädt, D., Möllmann, K. P., and Pinno, F., "Identification and Suppression of Thermal Reflections in Infrared Thermal Imaging," InfraMation 2004 Proceedings, Vol. 5, 2004, pp. 287-298.

[15] Holst, G. C., Holst's Practical Guide to Electro-Optical Systems, JCD Publishing, 2003.

[16] Davies, M. A., Yoon, H., Schmitz, T. L., Burns, T. J., and Kennedy, M. D., "Calibrated Thermal Microscopy of the Tool-Chip Interface in Machining," Machining Science and Technology, Vol. 7, No. 2, 2003, pp. 167-190.

[17] Baer, R. L., "Circular-edge spatial frequency response test," SPIE Proceedings, Vol. 5294, 2003, pp. 71-79.

[18] Boreman, G. D. and Yang, S., "Modulation Transfer Function Measurement Using Three- and Fourbar Targets," Supplement to Applied Optics, 1995, pp. 8050-8052.

[19] Nill, N. B., "Conversion Between Sine Wave and Square Wave Spatial Frequency Response of an Imaging System," MITRE Technical Report MTR01B0000021, July 2001.

[20] Hayat, M. M., Torres, S. N., Armstrong, E., Cain, S. C., and Yasuda, B., "Statistical Algorithm for Nonuniformity Correction in Focal-Plane Arrays," Applied Optics, Vol. 38, No. 8, 1999, pp. 772-780.

[21] Harris, J. G. and Chiang, Y., "Nonuniformity Correction of Infrared Image Sequences Using the Constant-Statistics Constraint," IEEE Transactions On Image Processing, Vol. 8, No. 8, 1999, pp. 1148-1151. 
[22] Cohen, M. and Cauwenberghs, G., "Floating-Gate Adaptation for Focal-Plane Online Nonuniformity Correction," IEEE Transactions On Circuits And Systems - II: Analog And Digital Signal Processing, Vol. 48, No. 1, 2001, pp. 83-89.

[23] Ratliff, B. M. and Hayat, M. M., "An Algebraic Algorithm for Nonuniformity Correction in FocalPlane Arrays," Journal of the Optical Society of America, Vol. 19, No. 9, 2002, pp. 1737-1747.

[24] Torres, S. N., Pezoa, J. E., and Hayat, M. M., "Scene-Based Nonuniformity Correction for Focal Plane Arrays by the Method of the Inverse Covariance Form," Applied Optics, Vol. 42, No. 29, 2003, pp. 5872-5881.

[25] Ratliff, B. M., Hayat, M. M., and Tyo, J. S., "Radiometrically Accurate Scene-Based Nonuniformity Correction for Array Sensors," Journal of the Optical Society of America, Vol. 20, No. 10, 2003, pp. 1890-1899.

[26] Zhou, H., Liu, S., Lai, R., Wang, D., and Cheng, Y., "Solution for the Nonuniformity Correction of Infrared Focal Plane Arrays," Applied Optics, Vol. 44, No. 15, 2005, pp. 2928-2932.

[27] Pezoa, J. E. and Hayat, M. M., "Multimodel Kalman Filtering for Adaptive Nonuniformity Correction in Infrared Sensors," Journal of the Optical Society of America, Vol. 23, No. 6, 2006, pp. 1282-1291.

[28] Shirley, E. L., "Revised Formulas for Diffraction Effects with Point and Extended Sources," Applied Optics, Vol. 37, No. 28, 1998, pp. 6581-6590.

[29] Whitenton, E. P., "Characterization of Uncertainties When Measuring Metal Cutting Temperatures Using Infrared Radiation Thermography," SPIE Proceedings, Vol. 7299, 2009, pp. 72990G-1-72990G12.

[30] Huang, G., Ni, G., and Zhang, B., "Visual and Infrared Dual-Band False Color Image Fusion Method Motivated by Land's Experiment," Optical Engineering, Vol. 46, No. 2, 2007, pp. 027001-1-10.

[31] Jin, H., Jiao, L., Liu, F., and Qi, Y., "Fusion of Infrared and Visual Images Based on Contrast Pyramid Directional Filter Banks Using Clonal Selection Optimizing," Optical Engineering, Vol. 47, No. 2, 2008, pp. 027002-1-8. 


\section{APPENDIX 1 - DIAGRAMS OF MADMACS.}

System components:

- Edgetek super-abrasive machining center: A CNC grinding machine with higher than normal horse power and stiffness.

- Kistler dynometer 3 axis force sensor: Used to measure cutting forces.

- Nicolet Odyssey digital signal oscilloscope: Records up to 8 analog and 8 digital high-speed signals to a computer hard drive.

- Push button: Used to manually trigger data acquisition. Normally, a relay in the Edgetek triggers data acquisition.

- Redington Counters model 83 timer: Once the command to start data acquisition has been issued, this timer starts the Nicolet first and then starts the thermal camera. The thermal camera starts the visible-light camera.

- Cedip Jade 3 thermal camera: A medium-speed MID-IR camera.

- Photron Fastcam-Ultima APX high-speed visible spectrum camera: This camera is not as fast as the one used for visible only measurements, but it is much faster than the thermal camera.

- DEI (Directed Energy Incorporated) PDG-2515 digital delay and pulse generator: Acts as the master clock for the thermal and highspeed visible light cameras.

- Trig-tek 36A ratio generator: Accepts a reference frequency signal and outputs another frequency which is the reference frequency times some user selected ratio.

- Sony camcorder: Once de-interlaced, the movies produced by this camcorder are 60 frames per second with an integration time of $16.7 \mathrm{~ms}$.

- Kramer VM19N RGB decoder: Extracts a once per frame pulse from the Sony camcorder. These pulses are recorded by the Nicolet Odyssey to help synchronize the camcorder movie to the rest of the data.

- Ametek Digistrobe III: A visible-light strobe sometimes used to make the effective integration time of the camcorder very short so that images produced by the camcorder have no motion blur. All other visible-light sources must be off for this to be effective.

- Aquastealth (ViaAqua) Polar Bear Chiller: Water chiller, modified by Aquastealth for use in cooling computer electronics.

- Little Giant sump and pump: Tank and pump to hold and circulate chilled water.

- Superior Electric Powerstat variable transformer (variac): A variable autotransformer used to control the voltage supplied to the chilled water pump. 


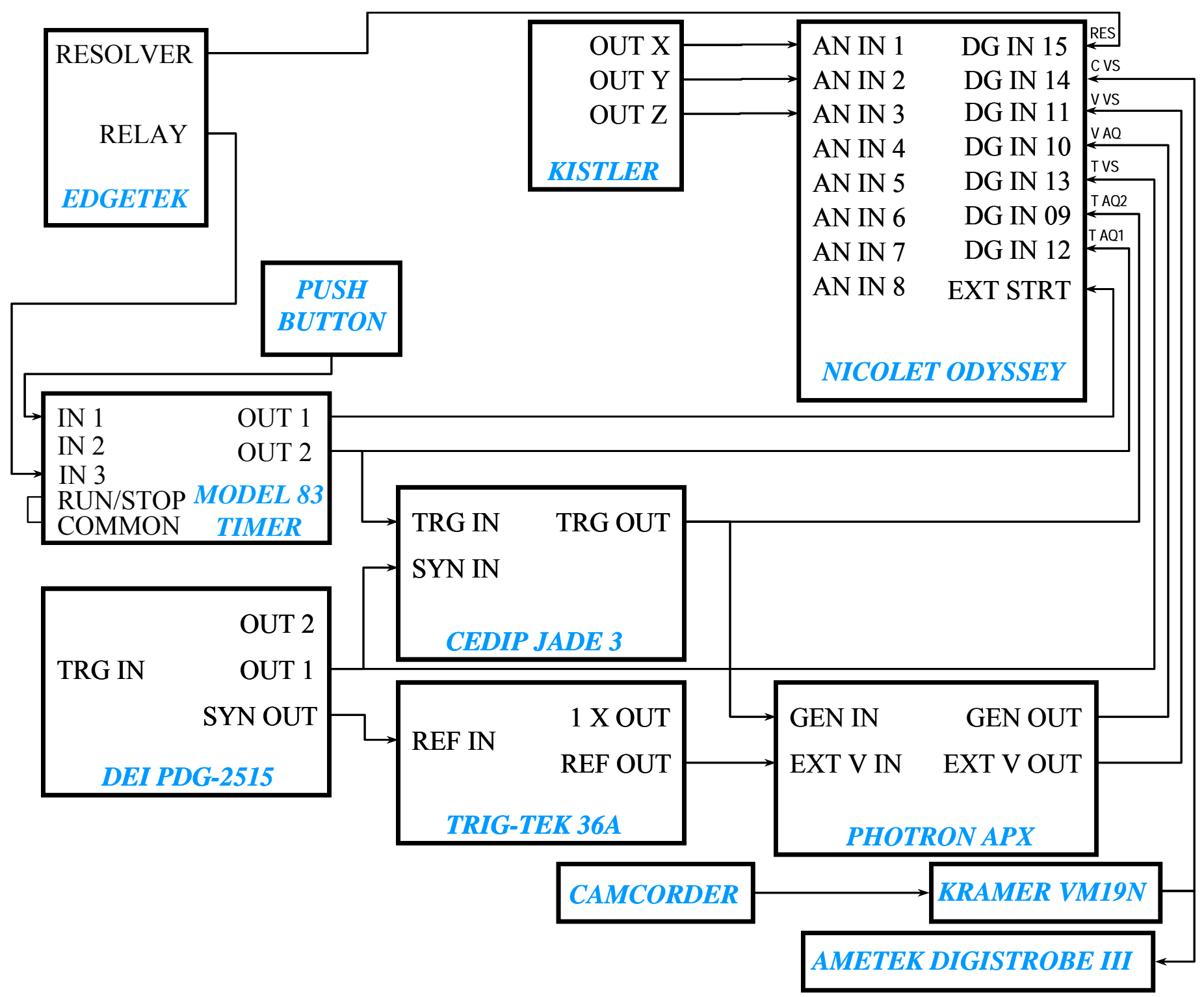

Figure A1.1. Data acquisition and control hardware interconnection diagram. 


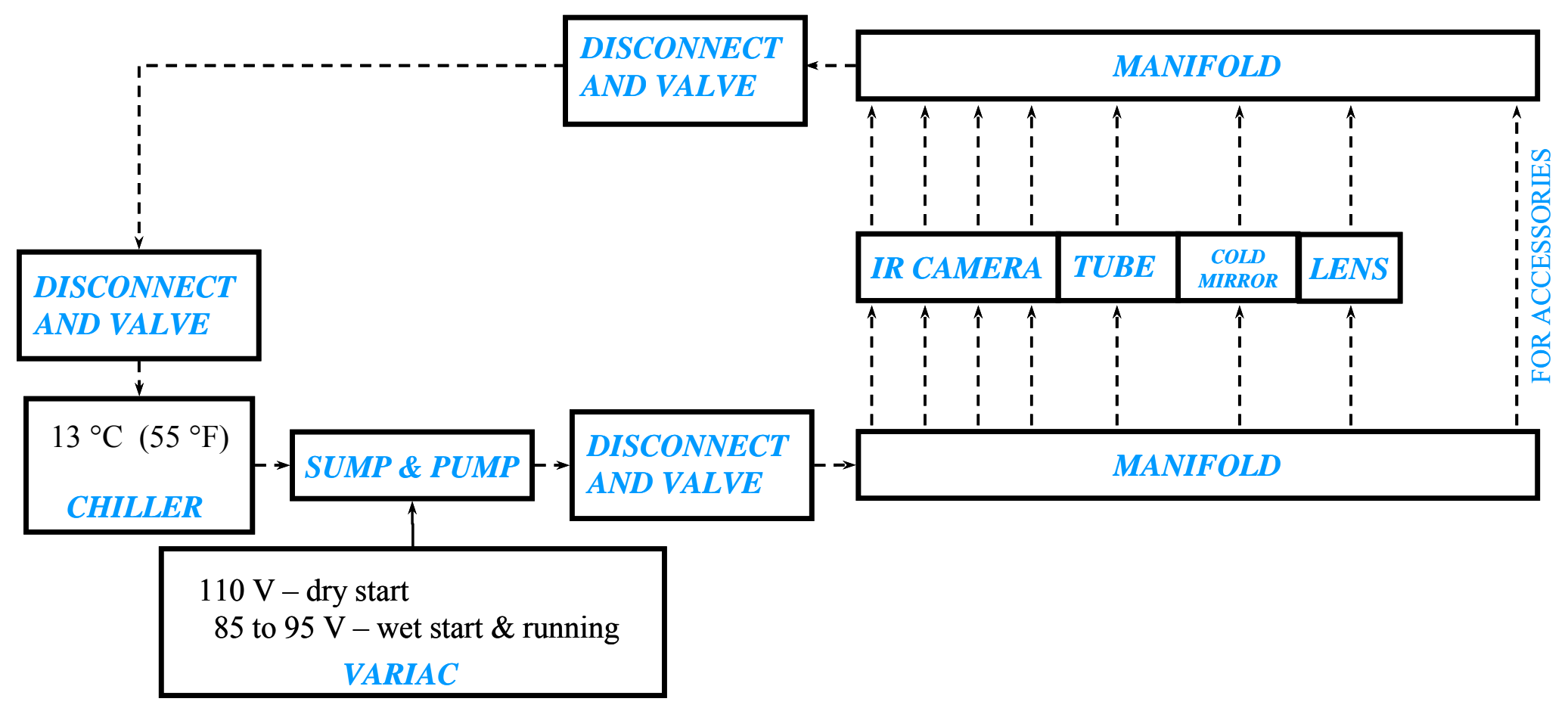

WATER

$\longrightarrow$ ELECTRICAL

Figure A1.2. Temperature control system diagram. 


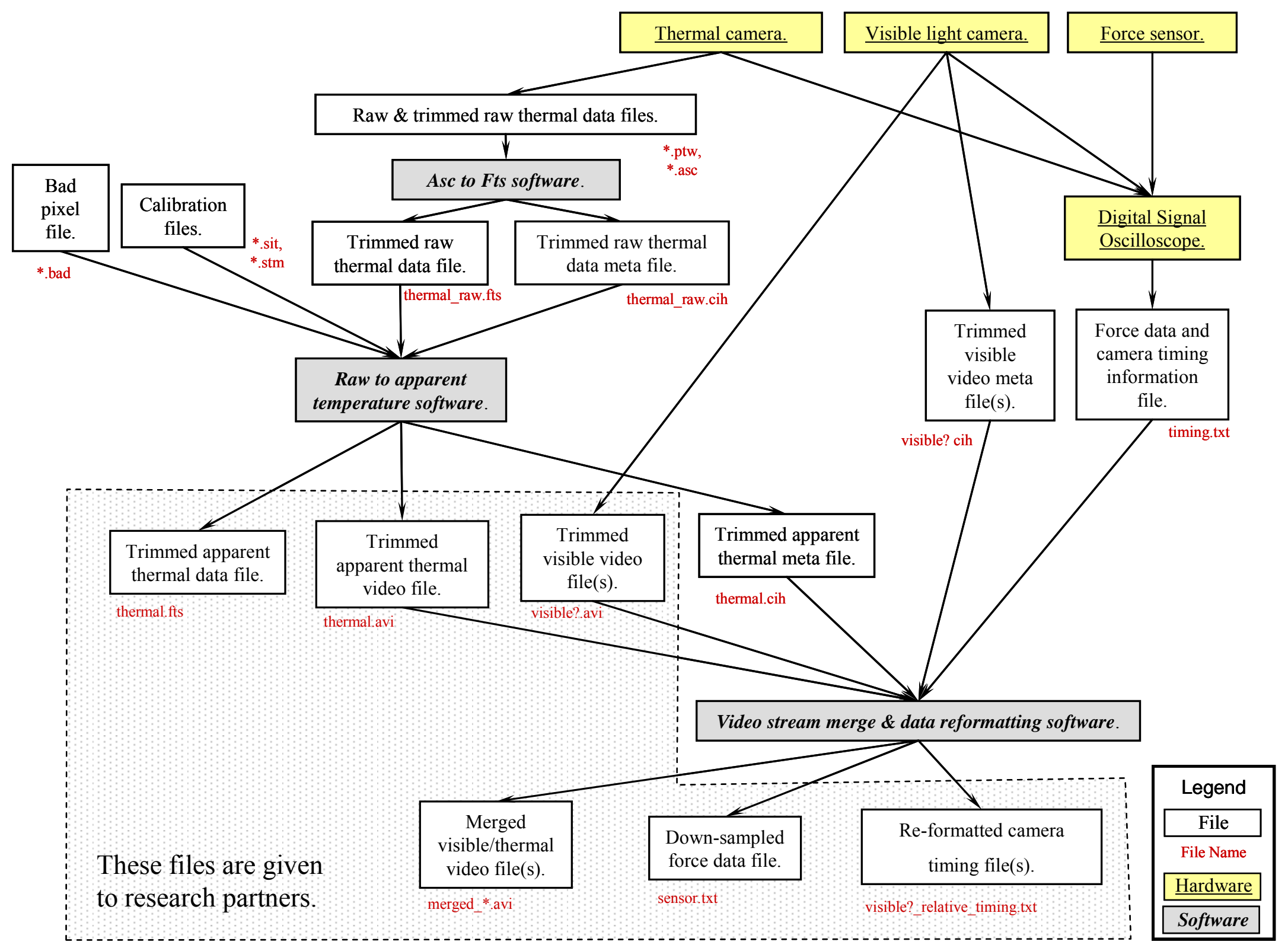

Figure A1.3. Data flow diagram. 


\section{APPENDIX 2 - NOMOGRAMS FOR MADMACS.}

A nomogram is a graph designed to allow both the approximate computation and uncertainty analysis of a function ${ }^{(7)}$. This appendix presents three nomograms for MADMACS, where each covers a different temperature range. They are based on Equation 5 in Example 6. To use the nomograms, first draw points at the locations of known quantities. Next, draw lines through those points. Finally, read unknown quantities determined where the drawn lines intersect some scale on the nomogram. The point corresponding to $T_{\text {environment }}$ is located on the left hand axis. Emissivity is the $\mathrm{X}$-coordinate and $T_{\text {apparent }}$ is the Y-coordinate of the point in the central portion of the nomogram. The point corresponding to $T_{\text {true }}$ is located on the right hand axis.

The nomogram on the right shows an example problem. Here, the effect of uncertainty in $\varepsilon$ on the uncertainty of the computed $T_{\text {true }}$ is determined. The effects of uncertainty in $T_{\text {apparent }}$ or $T_{\text {environment }}$ on the uncertainty of the computed $T_{\text {true }}$ could also be determined.

These nomograms work because the underlying equation

$$
I_{\text {apparent }}=\varepsilon \cdot I_{\text {true }}+(1-\varepsilon) \cdot I_{\text {environment }}
$$

is linear. Note that this equation uses intensities and not temperatures. It is only when each intensity is replaced with the temperature which corresponds to that intensity that the problem appears to be nonlinear.

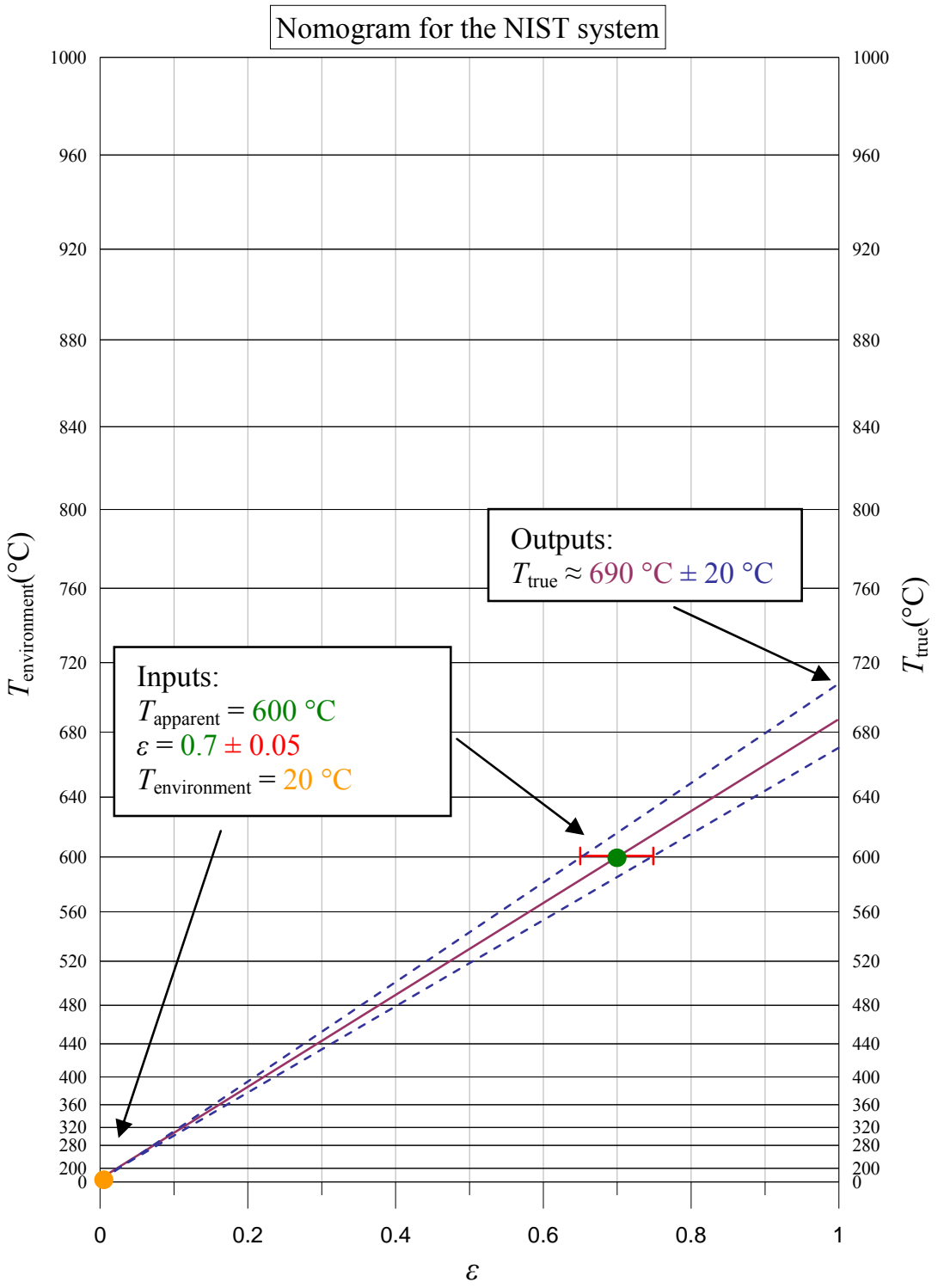

Figure A2.1. Example of how to use a nomogram. 


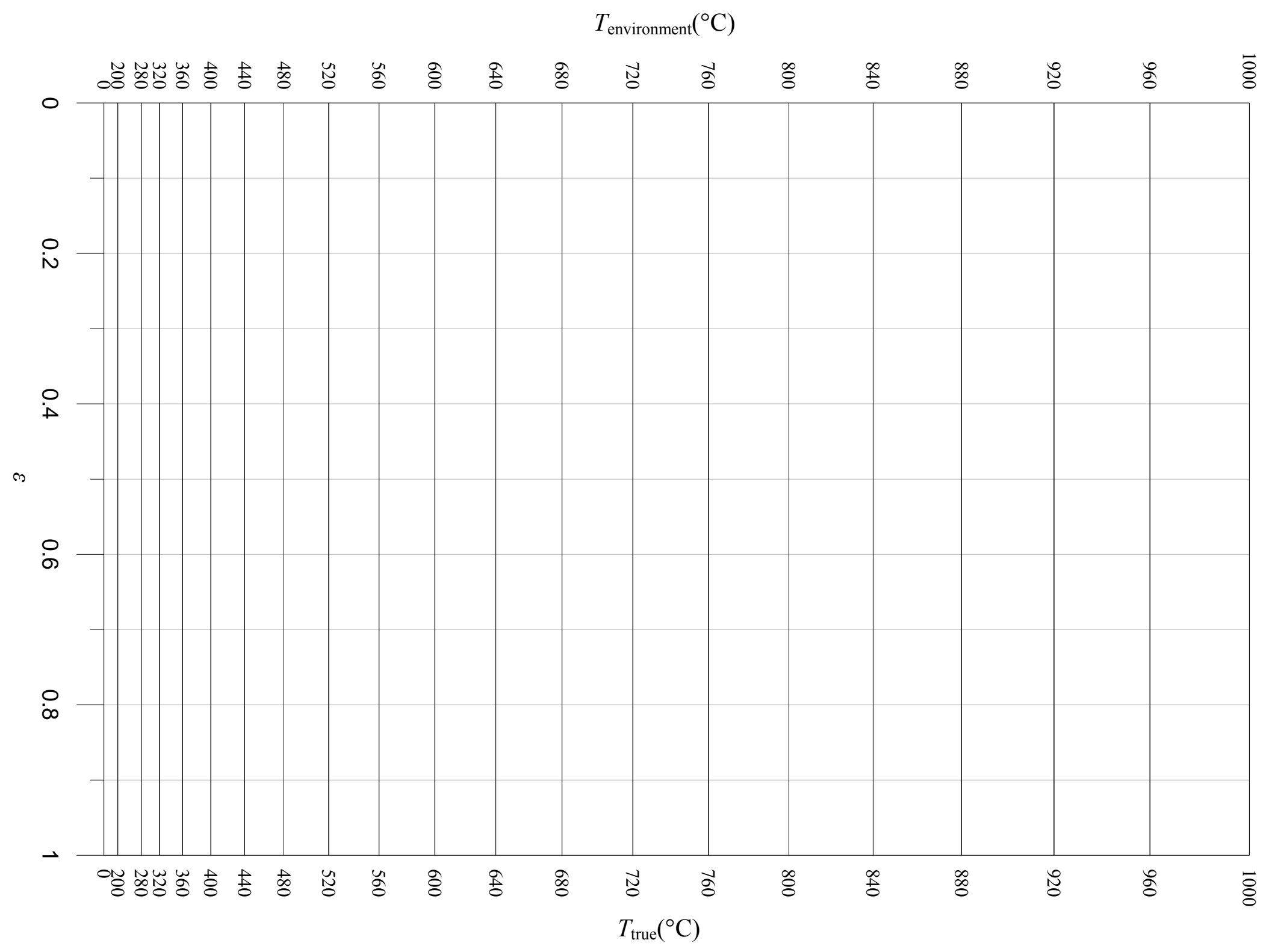

Figure A2.2. Nomogram for MADMACS for temperatures ranging from $0{ }^{\circ} \mathrm{C}$ to $1000{ }^{\circ} \mathrm{C}$. 


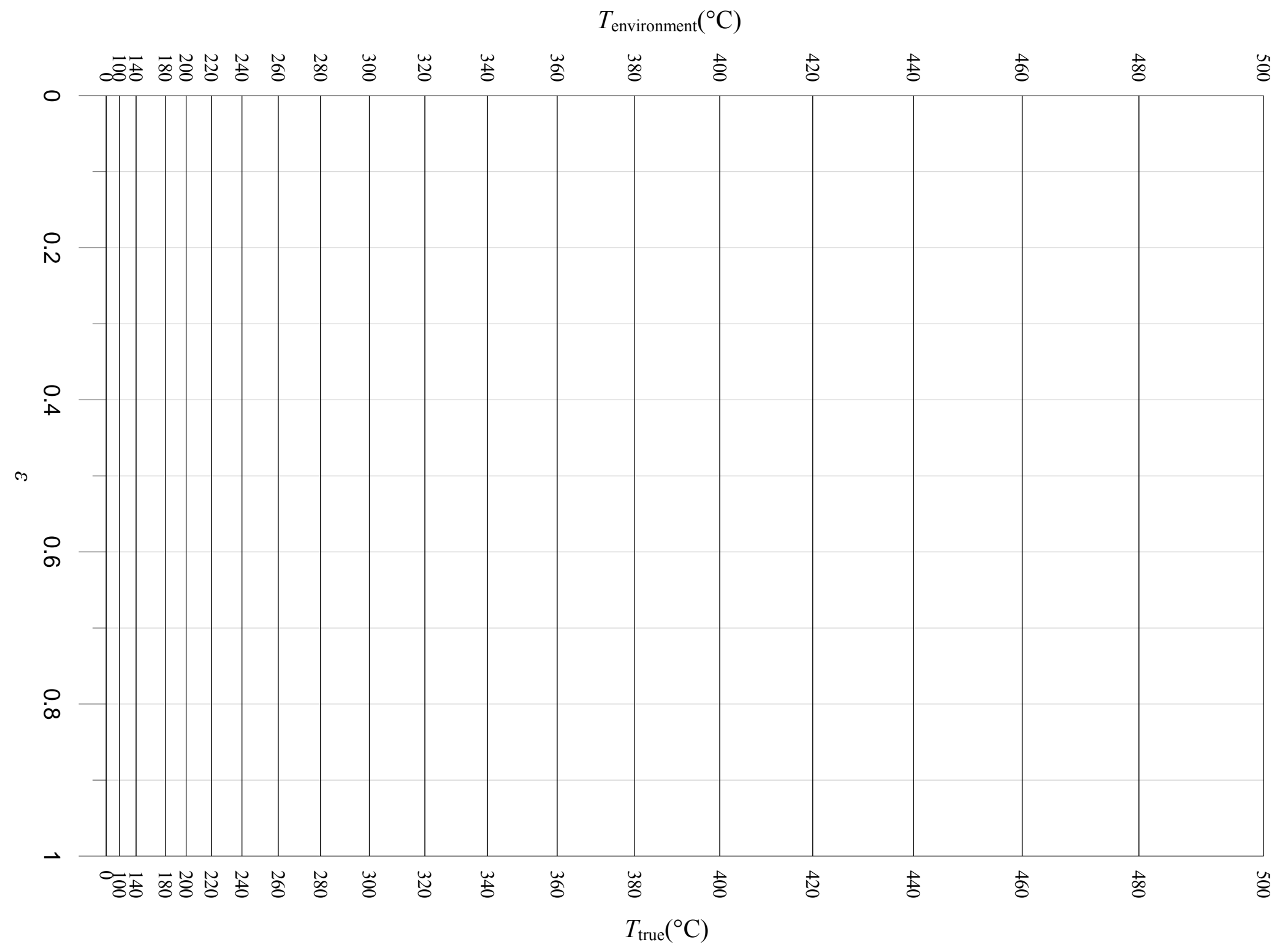

Figure A2.3. Nomogram for MADMACS for temperatures ranging from $0{ }^{\circ} \mathrm{C}$ to $500{ }^{\circ} \mathrm{C}$. 


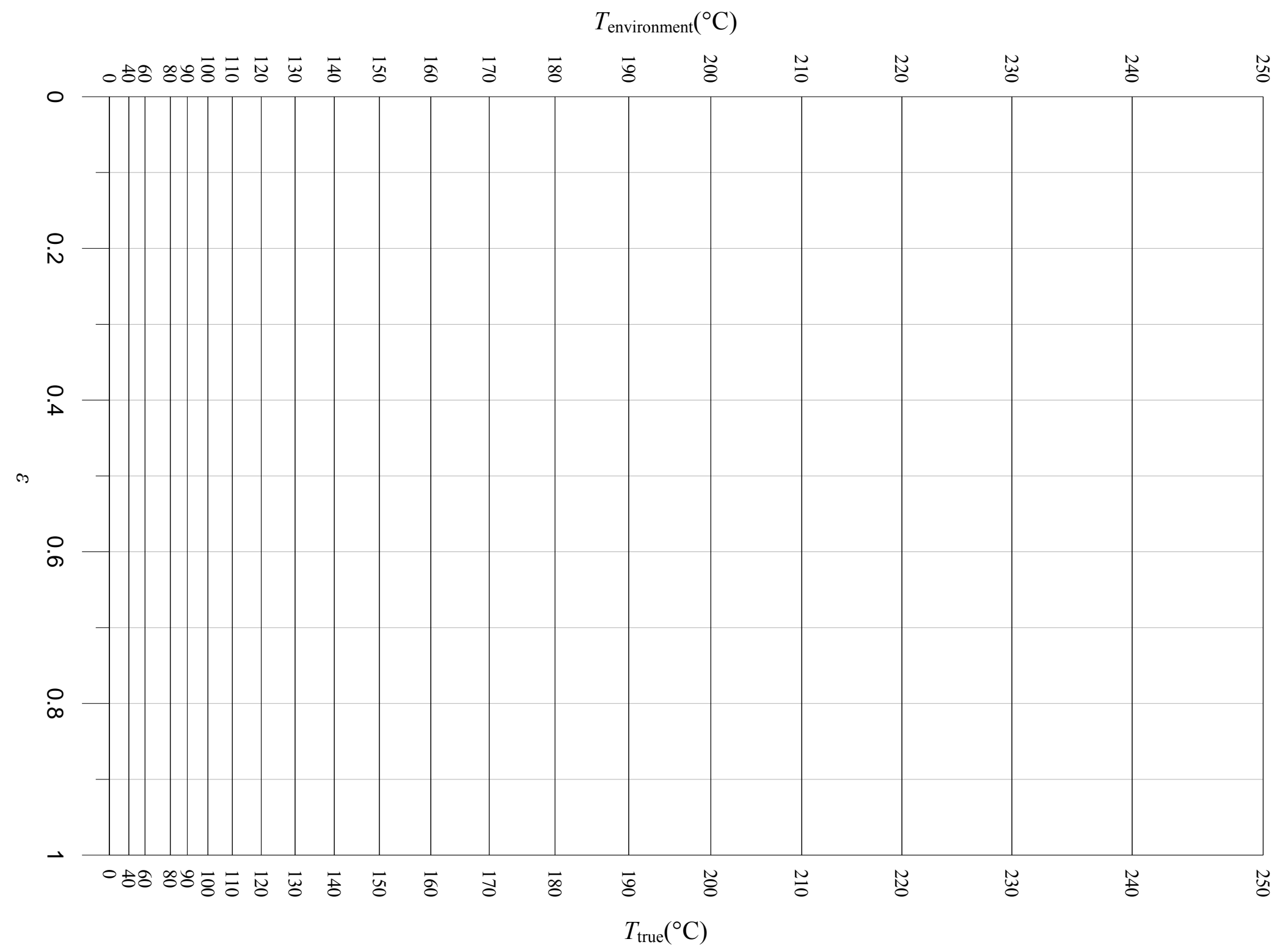

Figure A2.3. Nomogram for MADMACS for temperatures ranging from $0{ }^{\circ} \mathrm{C}$ to $250^{\circ} \mathrm{C}$. 


\section{APPENDIX 3 - MATHCAD ${ }^{\circledR}$ DOCUMENT TO COMPUTE ERROR DUE TO NONUNIFORM TEMPERATURE AND EMISSIVITY IN THERMAL IMAGES WHICH ARE MOTION BLURRED.}

This software was developed at the National Institute of Standards and Technology by employees of the Federal Government in the course of their official duties. Pursuant to title 17 Section 105 of the United States Code this software is not subject to copyright protection and is in the public domain. This is an experimental system. NIST assumes no responsibility whatsoever for its use by other parties, and makes no guarantees, expressed or implied, about its quality, reliability, or any other characteristic. We would appreciate acknowledgement if the software is used.

\section{Determining Error Due to Non-Uniform Temperature and Emis sivity in Thermal Images Eric Whitenton \\ NIST}

Second radiation constant $-\quad \mathrm{c} 2:=14388$

Offset to convert degrees $C$ to degrees $\mathrm{K}-\quad$ koff $:=273.15$

Mathcad tolerance constants - $\quad$ TOL $:=10^{-99} \quad$ CTOL $:=10^{-99}$

Range of lambda, in micrometers, the camera is sensitive to - $\quad \lambda 1:=3.80 \quad \lambda 2:=5.10$

Values will be integrated over the range of lambda using Simpsons rule- $n \lambda:=1001 \quad \mathrm{i}:=0 . . \mathrm{n} \lambda-1 \quad \lambda_{\mathrm{i}}:=\lambda 1+\frac{(\lambda 2-\lambda 1) \cdot \mathrm{i}}{\mathrm{n} \lambda-1}$

Range of temperatures, in degrees Centigrade, the camera images - $\quad \mathrm{T} 1:=500.00 \quad \mathrm{~T} 2:=900.00$

Values will be integrated over the range of temperatures using Simpsons rule- $n T:=51 \quad j:=0 . . n T-1 \quad T_{j}:=T 1+\frac{(T 2-T 1) \cdot j}{n T-1}$

Range of emissivities the camera images - $\quad \varepsilon 1:=0.01 \quad \varepsilon 2:=0.99$

Values will be integrated over the range of emissivities using Simpsons rule- $\quad \mathrm{n} \varepsilon:=51 \quad \mathrm{k}:=0 . . \mathrm{n} \varepsilon-1 \quad \varepsilon_{\mathrm{k}}:=\varepsilon 1+\frac{(\varepsilon 2-\varepsilon 1) \cdot \mathrm{k}}{\mathrm{n} \varepsilon-1}$

Create a weighting function for the various temperature,emissivity pairs -

$$
\mathrm{N}_{\mathrm{j}, \mathrm{k}}:=10 \cdot\left(\mathrm{dnorm}\left(\mathrm{T}_{\mathrm{j}}, 600, \frac{\mathrm{T} 2-\mathrm{T} 1}{10}\right) \cdot \operatorname{dnorm}\left(\varepsilon_{\mathrm{k}}, 0.3, \frac{\varepsilon 2-\varepsilon 1}{10}\right)\right)+1\left(\operatorname{dnorm}\left(\mathrm{T}_{\mathrm{j}}, 800, \frac{\mathrm{T} 2-\mathrm{T} 1}{15}\right) \cdot \operatorname{dnorm}\left(\varepsilon_{\mathrm{k}}, 0.7, \frac{\varepsilon 2-\varepsilon 1}{15}\right)\right)
$$


Insure the weighting function is properly normalized -

$$
\mathrm{sN}:=\sum_{\mathrm{j}} \sum_{\mathrm{k}} \mathrm{N}_{\mathrm{j}, \mathrm{k}} \quad \mathrm{N}_{\mathrm{j}, \mathrm{k}}:=\frac{\mathrm{N}_{\mathrm{j}, \mathrm{k}}}{\mathrm{sN}}
$$

Compute unitless intensities the camera sees for the various temperature,emissivity pairs -

$I_{j, k}:=N_{j, k} \cdot \frac{\sum_{i} \frac{\varepsilon_{k}}{\left(\lambda_{i}\right)^{5} \cdot\left[e^{\frac{c 2}{\lambda_{i j}\left(T_{j}+\text { koff }\right)}}-1\right]}}{n \lambda}$

Compute average temperature, average emissivity and average intensity - aT $:=\sum_{j} \sum_{k}\left(\mathrm{~N}_{\mathrm{j}, \mathrm{k}} \cdot \mathrm{T}_{\mathrm{j}}\right)$

$$
\begin{aligned}
& \mathrm{a} \varepsilon:=\sum_{\mathrm{j}} \sum_{\mathrm{k}}\left(\mathrm{N}_{\mathrm{j}, \mathrm{k}} \cdot \varepsilon_{\mathrm{k}}\right) \\
& \mathrm{SI}:=\sum_{\mathrm{j}} \sum_{\mathrm{k}}\left(\mathrm{I}_{\mathrm{j}, \mathrm{k}}\right)
\end{aligned}
$$

Create variables to help plot results - $\quad \mathrm{NN}(\mathrm{j}, \mathrm{k}):=\left(\begin{array}{c}\mathrm{T}_{\mathrm{j}} \\ \varepsilon_{\mathrm{k}} \\ \mathrm{N}_{\mathrm{j}, \mathrm{k}}\end{array}\right) \quad \mathrm{NNN}:=$ CreateMesh $(\mathrm{NN}, 0, \mathrm{nT}-1,0, \mathrm{n} \varepsilon-1, \mathrm{nT}, \mathrm{n} \varepsilon)$
$\mathrm{II}(\mathrm{j}, \mathrm{k}):=\left(\begin{array}{c}\mathrm{T}_{\mathrm{j}} \\ \varepsilon_{\mathrm{k}} \\ \left.\mathrm{I}_{\mathrm{j}, \mathrm{k}}\right)\end{array} \quad \mathrm{III}:=\right.$ CreateMesh $(\mathrm{II}, 0, \mathrm{nT}-1,0, \mathrm{n} \varepsilon-1, \mathrm{nT}, \mathrm{n} \varepsilon)$ 


$$
\begin{aligned}
& \text { given } \\
& \mathrm{Q}(\mathrm{ttt}):=\left|\mathrm{sI}-\left[\frac{\left.\sum_{\mathrm{i}} \frac{\mathrm{a} \varepsilon}{\left(\lambda_{\mathrm{i}}\right)^{5} \cdot\left[\mathrm{e}^{\frac{\mathrm{c} 2}{\lambda_{\mathrm{i}(\mathrm{tt}+\mathrm{koff})}}-1}\right.}\right]}{\mathrm{n} \lambda}\right]\right| \\
& \mathrm{pT}:=\operatorname{Minimize}(\mathrm{Q}, \mathrm{aT})
\end{aligned}
$$

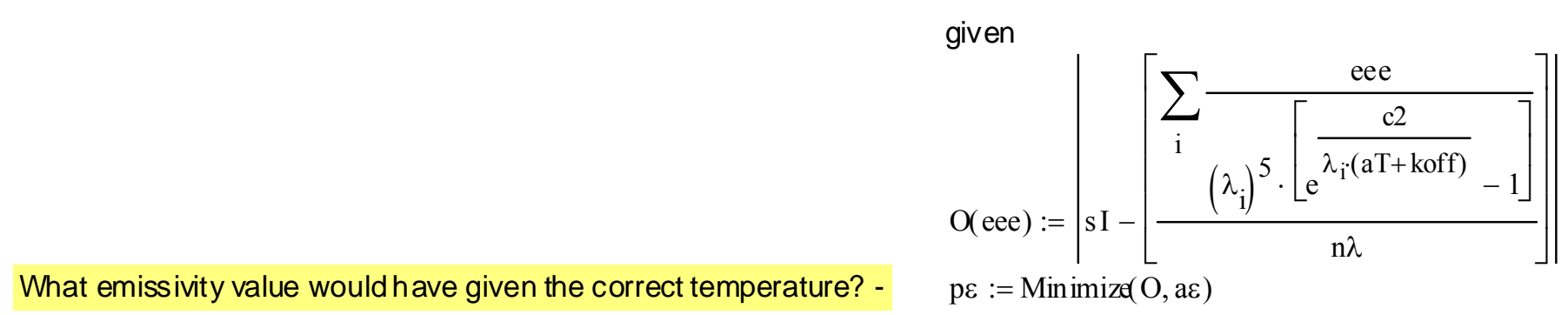




\section{RESULTS}
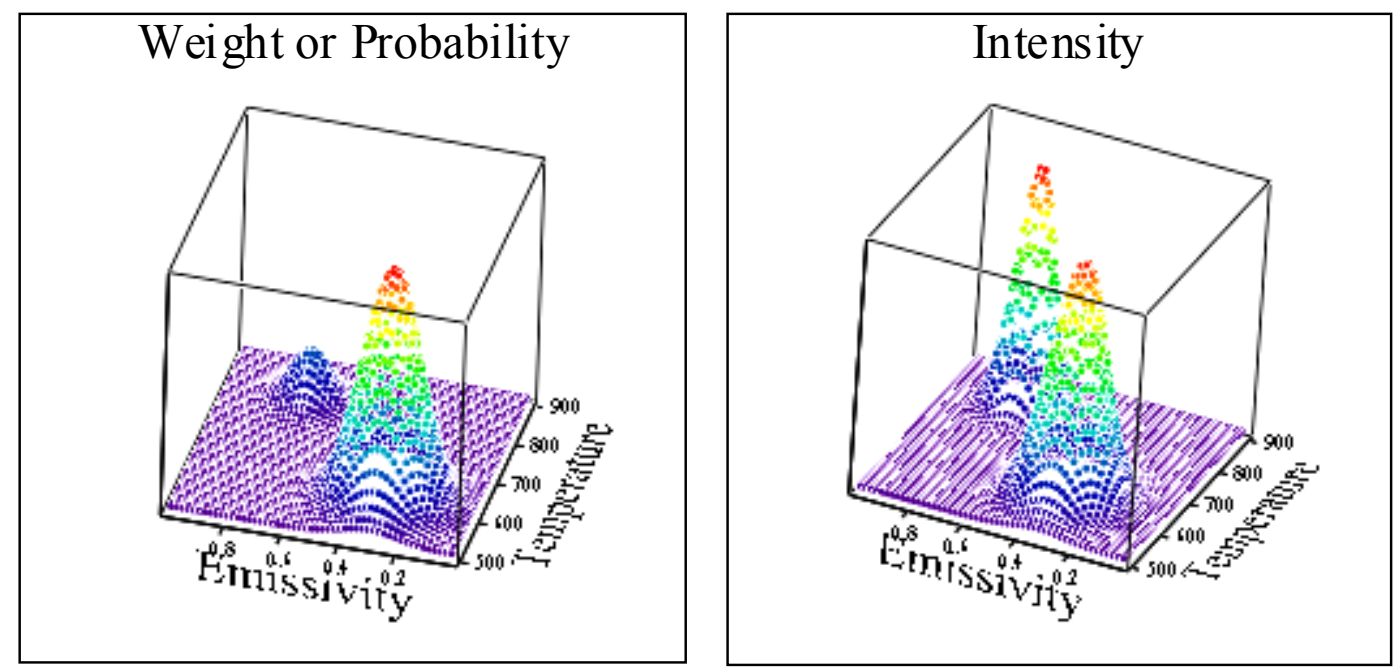

Actual average temperature (in degrees Centigrade) -

$\mathrm{aT}=618.766$

Apparent average temperature (in degrees Centigrade) -

$\mathrm{pT}=645.669$

Difference (in degrees Centigrade) -

$\mathrm{pT}-\mathrm{aT}=26.902$

Actual average emissivity -

$\mathrm{a} \varepsilon=0.337$

Emissivity value which would have given correct temperature- $p \varepsilon=0.376$

Difference -

$\mathrm{p} \varepsilon-\mathrm{a} \varepsilon=0.04$ 


\section{APPENDIX 4 - MATHCAD ${ }^{\circledR}$ DOCUMENT TO CORRECT SHEAR BAND TEMPERATURE MEASUREMENTS.}

This software was developed at the National Institute of Standards and Technology by employees of the Federal Government in the course of their official duties. Pursuant to title 17 Section 105 of the United States Code this software is not subject to copyright protection and is in the public domain. This is an experimental system. NIST assumes no responsibility whatsoever for its use by other parties, and makes no guarantees, expressed or implied, about its quality, reliability, or any other characteristic. We would appreciate acknowledgement if the software is used.

\section{Correcting for Motion Bitur and PSF in Thermal Measurement of a Shear Band \\ Eric Whitenton \\ NIST}

\section{Note that some variables are defined globally at the bottom of this document!}

\section{Second radiation constant $-\quad \mathrm{c} 2:=14388$}

Offset to convert degrees $\mathrm{C}$ to degrees $\mathrm{K}-\quad$ koff $:=273.15$

Mathcad tolerance constants - TOL $:=10^{-99} \quad \mathrm{CTOL}:=10^{-99}$

Range of lambda, in micrometers, the camera is sensitive to $-\quad \lambda 1:=3.80 \quad \lambda 2:=5.10$

Values will be integrated over the range of lambda using Simpsons rule- $\mathrm{n} \lambda:=1001$

$i:=0 . . n \lambda-1 \quad \lambda_{i}:=\lambda 1+\frac{(\lambda 2-\lambda 1) \cdot i}{n \lambda-1}$

Temperatue values in degrees $\mathrm{C}$ -

$\mathrm{T}:=$

$\mathrm{nT}:=\operatorname{rows}(\mathrm{T})$

$\mathrm{j}:=0 . . \mathrm{nT}-1$

C...,thermal-data.txt 
Compute unitless intensity data with baseline removed -

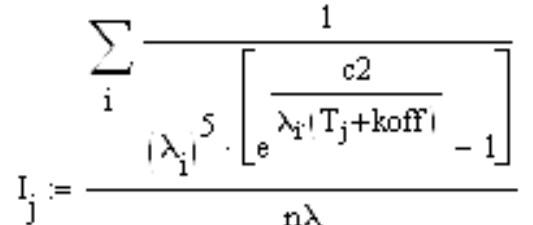

n

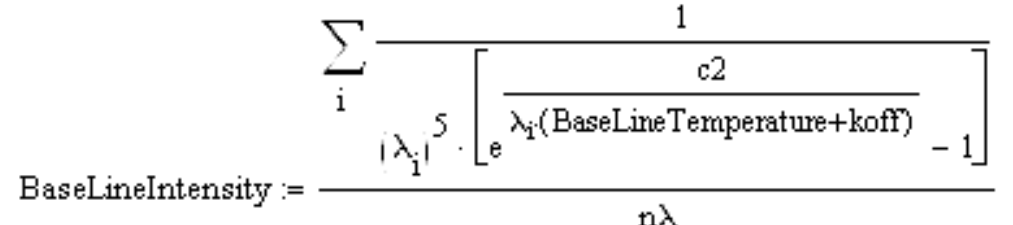

$\mathrm{n} \lambda$

$I_{j}:=I_{j}-$ BaseLineIntensity

Model intensity data as Sinc Function. This will not work for some data sets. There are several reasons to model intensity as a Sinc Function. The primary reason is that it facilitates alignment (in X) of the intensity data to the results of the convolution.

Estimate parameters for Sinc Function fit of intensity data -

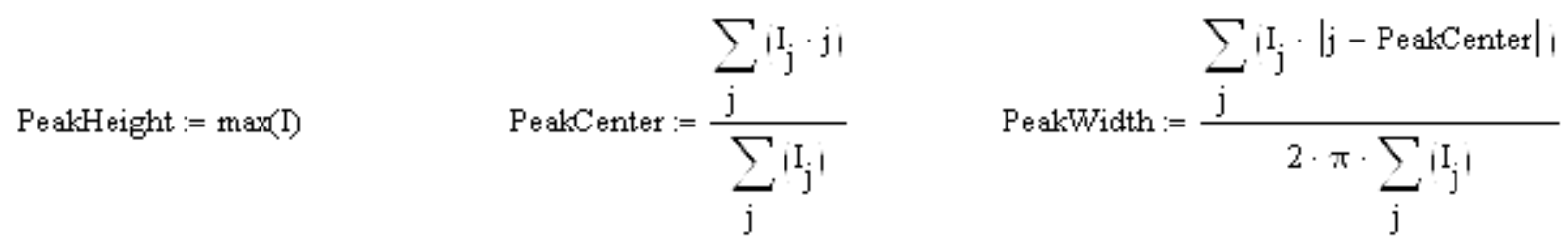

Perform fit of Sinc Function to intensity data -

$$
\begin{aligned}
& X_{j}:=j \quad G:=\left(\begin{array}{c}
\text { PeakHeight } \\
\text { PeakCenter } \\
\text { PeakWidth }
\end{array}\right) \quad F(x, a, b, c):=a \cdot \operatorname{sinc}[c \cdot(x-b)] \quad G:=\operatorname{genfit}(X, I, G, F) \\
& \text { PeakHeight }:=G_{0} \quad \text { PeakCenter }:=G_{1} \quad \text { PeakWidth }:=G_{2}
\end{aligned}
$$

Resample (and extend and center) data - 
Get camera point spread function (right half of) from disk file. Goes from 1 to 0 -

For convience, the PSF is centered in the data set while the other convolution terms are centered around time 0

$$
\begin{aligned}
& \mathrm{psp}:= \\
& \text { 回 } \\
& \mathrm{nP}:=\text { rows }(\mathrm{psp}) \\
& \mathrm{p}:=0 . . \mathrm{nP}-1
\end{aligned}
$$

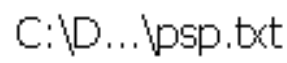

$$
\begin{aligned}
& \mathrm{vx}_{\mathrm{p}}:=\mathrm{p} \quad \mathrm{vs}:=1 \mathrm{spline}(\mathrm{vx}, \mathrm{psp}) \\
& \mathrm{fTemp}(\mathrm{kk}):=\mid \begin{array}{ll}
0 & \text { if } \mathrm{kk}>\mathrm{nP} \\
\operatorname{interp}(\mathrm{vs}, \mathrm{vx}, \mathrm{psp}, \mathrm{kk}) & \text { otherwise }
\end{array} \\
& \operatorname{ResPSF}_{k}:=f T e m p\left(\left|\frac{k-\text { ResOff }}{\text { ResScale }}\right|\right)
\end{aligned}
$$

Normalize point spread function of camera -

$$
\text { Temp }:=\sum_{\mathrm{k}} \operatorname{ResPSF}_{\mathrm{k}} \quad \operatorname{ResPSF}_{\mathrm{k}}:=\frac{\operatorname{ResPSF}_{\mathrm{k}}}{\mathrm{Temp}}
$$

Normalized motion blur bar -

$$
f \operatorname{emp}(\mathrm{kk}):=\mid \begin{aligned}
& 1 \text { if } \mathrm{kk}<\frac{\text { MotionBlurWidth }}{2} \\
& 1 \text { if } \frac{\mathrm{Nk}}{\text { ResScale }}-\mathrm{kk}<\frac{\text { MotionBlurWidth }}{2} \\
& 0 \text { otherwise }
\end{aligned} \quad \text { ResMotionBlut }{ }_{\mathrm{k}}:=\operatorname{fTemp}\left(\frac{\mathrm{k}}{\text { ResScale }}\right)
$$$$
\text { Temp }:=\sum_{\mathrm{k}} \operatorname{ResMotionBlut}_{\mathrm{k}}
$$$$
\text { ResMotionBlux }_{k}:=\frac{\text { ResMotionBlux }_{k}}{\text { Temp }}
$$ 
Convolve fit of measured data with shear zone "bar" of unit height with motion blur and camera response to determine intensity of shear zone bar -

Factors to correct for the way Mathcad scales the cff and icft functions -

$$
\text { cffScale }:=\sqrt{\mathrm{Nk}} \quad \text { icffScale }:=\frac{1}{\sqrt{\mathrm{Nk}}}
$$

Width in pixels of shear zone -

$$
f \operatorname{ermp}(\mathrm{kk}):=\mid \begin{array}{ll}
1 & \text { if } \mathrm{kk}<\frac{\text { ShearZoneWidth }}{2} \\
1 & \text { if } \frac{\mathrm{Nk}}{\text { ResScale }}-\mathrm{kk}<\frac{\text { ShearZoneWidth }}{2} \\
0 \text { otherwise }
\end{array}
$$

$$
\text { ResShearZone }_{\mathrm{k}}:=\mathrm{fTemp}\left(\frac{\mathrm{k}}{\operatorname{ResScale}}\right)
$$

Convolve -

$$
\begin{array}{ll}
\mathrm{T} 1:=\operatorname{cffScale} \cdot \operatorname{cff}(\text { ResPSF }) & \mathrm{T} 2:=\operatorname{cffScale} \cdot \operatorname{cff}(\text { ResMotionBlut) } \\
\mathrm{T} 4_{\mathrm{k}}:=\mathrm{T} 1_{\mathrm{k}} \cdot \mathrm{T} 2_{\mathrm{k}} \cdot \mathrm{T} 3_{\mathrm{k}} \quad \text { ResIConvoluted }:=\mathrm{icffScale} \cdot \operatorname{icft}(\mathrm{T} 4)
\end{array}
$$

Re-generate shear zone bar at correct amplitude-

$$
\begin{aligned}
& \text { ResOff }+\frac{\text { Nk }}{2 \text { ResExt }}
\end{aligned}
$$

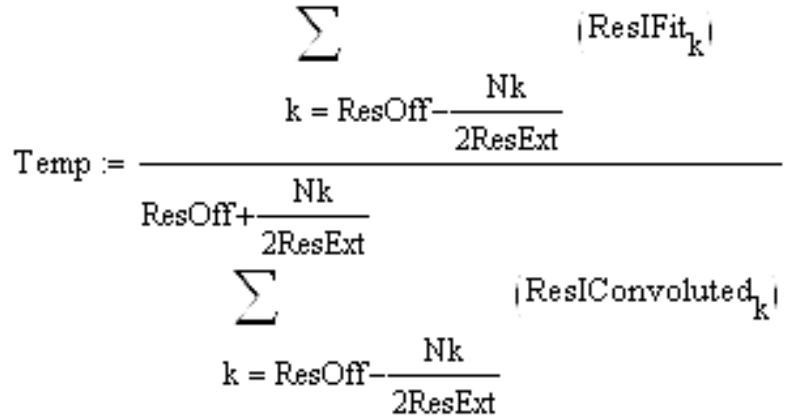


Recompute convolution -

$\mathrm{T} 3:=\operatorname{cftScale} \cdot \operatorname{cft}($ ResShearZone)

$$
\mathrm{T} 4_{\mathrm{k}}:=\mathrm{T} 1_{\mathrm{k}} \cdot \mathrm{T} 2_{\mathrm{k}} \cdot \mathrm{T} 3_{\mathrm{k}}
$$

ResIConvoluted $:=$ icftScale $\cdot \operatorname{icfft}(\mathrm{T} 4)$

Add baseline intensity back in -

ShearZoneIntensity $:=\max ($ ResShearZone) + BaseLineIntensity

ResIFit := ResIFit + BaseLineIntensity

ResIConvoluted $:=$ ResIConvoluted + BaseLineIntensity

Determine residuals in area of original (non-extended) data-

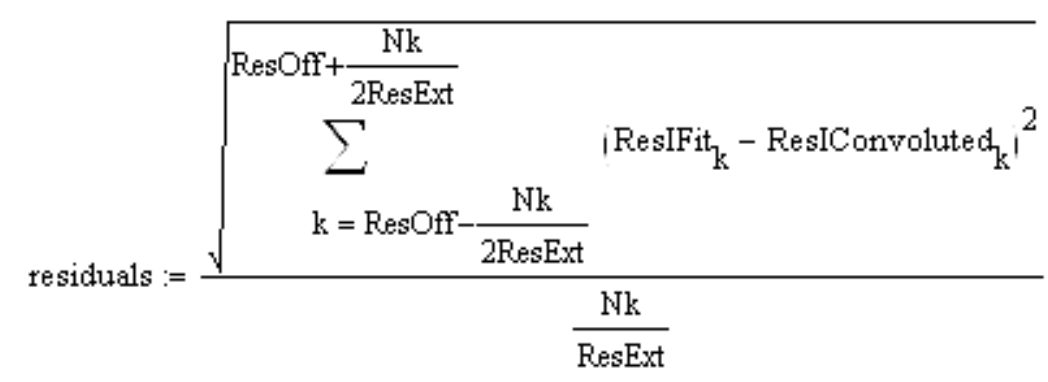

Determine shear zone temperature -

Use maximum temperature measured as initial guess -

MaxTemperatureMeasured $:=\max (T)$

$Q(t t r):=\mid$ ShearZoneIntensity $-\left[\frac{\left.\sum_{i} \frac{1}{\left|\lambda_{i}\right|^{5} \cdot\left[e^{\frac{c 2}{\lambda_{i}(t t t+k o f f)}}-1\right.}\right]}{n \lambda}\right] \mid$

ShearZoneTemperature $:=$ Minimize(Q, MaxTemperatureMeasured) 


\section{RESULTS}
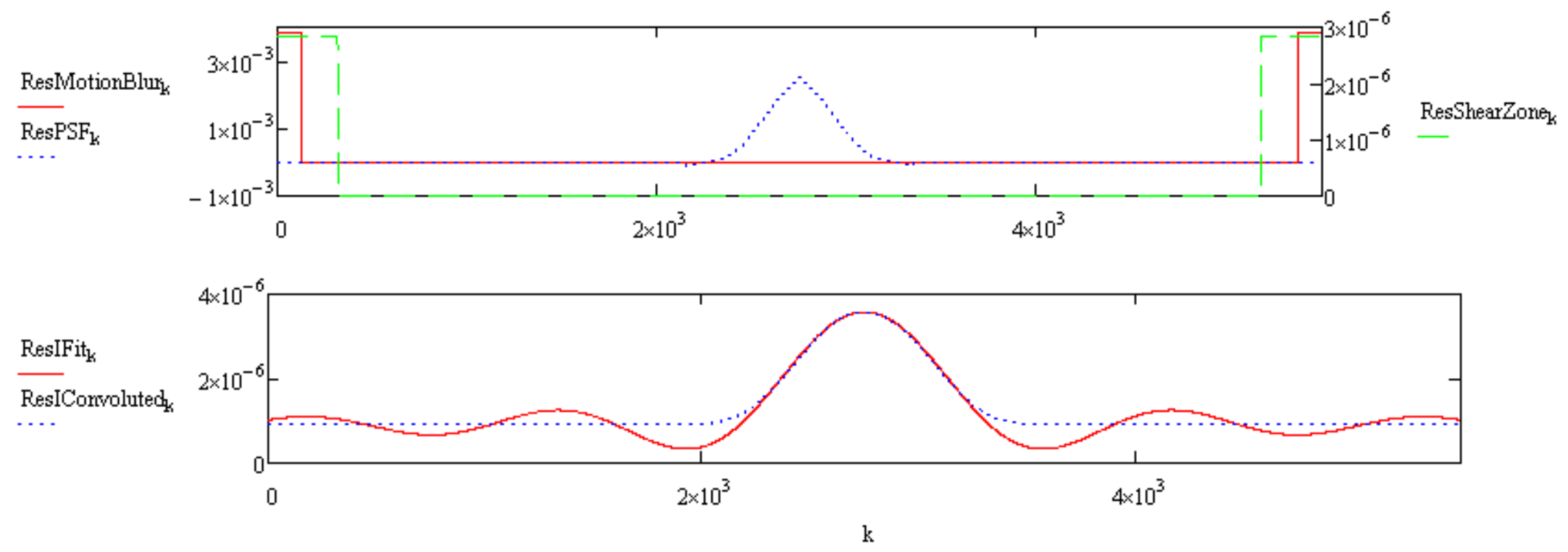

\section{INPUTS}

Baseline temperature in degrees C-

Shear zone width in pixels -

Width in pixels of blur due to motion-

Rescale \# data points, more accuracy -

Extend data set so all of function

included-

\section{OUTPUTS}

BaseLineT emperature $\equiv 230$

ShearZoneWidth $\equiv 6.5$

MotionBlurWidth $\equiv 2.6$

ResScale $\equiv 100$

ResExt $\equiv 5$
Fit error in arb units -

residuals $=1.556 \times 10^{-9}$

Max temperature measured degrees $\mathrm{C}-$ MaxT emperatureMeasured $=365.7$

Shear zone temperature in degrees $\mathrm{C}-\quad$ ShearZoneT emperature $=371.413$ 
